
CRPL-F 242 PART A
NATIONAL BUREAU OF STANDARDS CENTRAL RADIO PROPAGATION LABORATORY BOULDER, COLORADO issect

30 Det. 1961

\section{IONOSPHERIC DATA}

\section{CONTENTS}

$\underline{\text { Page }}$

Ionospheric Data . . . . . . . . . . ii

Table of Smoothed Observed Zurich Sunspot Numbers . iii World-Wide Sources of Ionospheric Data . . . . . iv Tables and Graphs of Ionospheric Data ....... 1 Index of Tables and Graphs of Ionospheric Data in CRPL-F242 (Part A) . . . . . . 51 
The CRPL-F series bulletins are issued as part of the responsibility of the Central Radio Propagation Laboratory for the exchange and distribution of ionospheric and related geophysical data. Part A, "Ionospheric Data," and Part B, "Solar-Geophysical Data," of the CRPL-F series present a variety of data collected by CRPL in the course of its research and service activities. Through the CRPL-F series, as part of the general exchange of scientific information, these data are made available for use by others in research on radio propagation and the ionosphere, and in other geophysical applications.

In the CRPL-F series, Part $A$, tables of monthly median values of vertical-incidence ionospheric data are presented accompanied by graphs of critical frequencies and $M(3000) F 2$. The tables include the number of values entering into the median determination (count). When available, the upper and lower quartile values (indicated by UQ and LQ) are 1 isted for foF 2, foFl, foEs, M(3000)F2, h'F2 and h'F. Space 1 imitations do not permit inclusion of quartile values for the other characteristics. The tables are prepared by machine methods and the graphs are plotted automatically.

The tables and graphs present the ionospheric data as received from the originating laboratory. Responsibility for the accuracy and reliability of the data rests entirely with the originator. Medians of data for the U.S. stations are computed by CRPL in accordance with the recommendations of the World-Wide Soundings Comittee.

Data will appear in the F-series, Part A, only when the complete daily-hourly tabulations have been received by the CRPL or the World Data Center A for Airglow and Ionosphere. In general, priority of publication is given to the most current data. Data received too long after the month of observation may experience an indefinitely prolonged delay before finding space in the $\mathrm{F}$ series, Part A.

Information on symbols, terminology and conventions may be found in the "URSI Handbook of Ionogram Interpretation and Reduction of the World-Wide Soundings Committee," edited by W. R. Piggott and K. Rawer (Elsevier, 1961), which supersedes previous documents. A 1ist of symbols is available from CRPL on request.

\section{Units and Abbreviations of Ionospheric Data Tables}

foF 2, foEs - - Tenths of a megacycie

foFl, foE - - Hundredths of a megacycle

$h^{\prime} F 2, h^{\prime} F, h^{\prime} E-K i l o m e t e r s$

$\mathrm{M}(3000) \mathrm{F} 2$ - Hundredths
MED - Median

CNT - Count

UQ - Upper Quartile

LQ - Lower Quartile 
Key to Points of Ionospheric Data Graphs

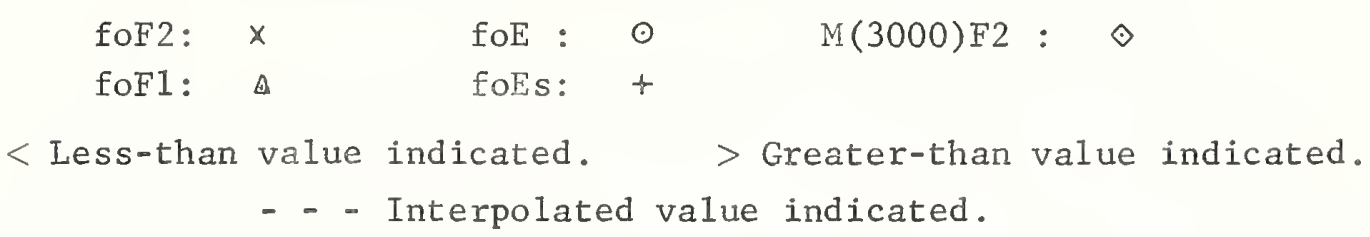

The following table contains the latest available information on twelve-month smoothed average of observed Zurich relative sunspot numbers, beginning with the minimum of Apri1 1954. Final numbers are listed through June 1963, the succeeding values being based on provisional data.

Smoothed Observed Zurich Relative Sunspot Number

\begin{tabular}{lrrrrrrrrrrrr}
\hline Month & Jan. Feb. Mar. Apr. May & Jun. Jul. Aug. Sep. Oct. & Nov. Dec. \\
\hline 1954 & & & & 3 & 4 & 4 & 5 & 7 & 8 & 8 & 10 & 12 \\
1955 & 14 & 16 & 19 & 23 & 29 & 35 & 40 & 46 & 55 & 64 & 73 & 81 \\
1956 & 89 & 98 & 109 & 119 & 127 & 137 & 146 & 150 & 151 & 156 & 160 & 164 \\
1957 & 170 & 172 & 174 & 181 & 186 & 188 & 191 & 194 & 197 & 200 & 201 & 200 \\
1958 & 199 & 201 & 201 & 197 & 191 & 187 & 185 & 185 & 184 & 182 & 181 & 180 \\
1959 & 179 & 177 & 174 & 169 & 165 & 161 & 156 & 151 & 146 & 141 & 137 & 132 \\
1960 & 129 & 125 & 122 & 120 & 117 & 114 & 109 & 102 & 98 & 93 & 88 & 84 \\
1961 & 80 & 75 & 69 & 64 & 60 & 56 & 53 & 52 & 52 & 51 & 50 & 49 \\
1962 & 45 & 42 & 40 & 39 & 39 & 38 & 37 & 35 & 33 & 31 & 30 & 30 \\
1963 & 29 & 30 & 30 & 29 & 29 & 28 & 28 & 27 & 27 & 26 & 23 & 21 \\
1964 & 19 & 17 & 15 & & & & & & & & &
\end{tabular}

\section{NOTICE OF CHANGE OF FORMAT}

Beginning with this issue, tables and graphs of ionospheric data will appear on the same page side by side, with two station months of data summary tables and their corresponding graphs on a single page. The same number of station months of data will appear per issue. The change in proportion of the graphs permits somewhat larger printing for the tabulated data, resulting in more legible tables. We believe that most readers will also find the new arrangement more convenient to use. 
THE IONOSPHERIC DATA PRESENTED IN THE 100 TABLES AND GRAPHS OF THIS ISSUE WERE ASSEMBLED BY THE CENTRAL RADIO PROPAGATION LABORATORY FOR ANALYSIS, CORRELATION, AND

DISTRIBUTION. THE FOLLOWING ARE THE SOURCES OF THE DATA:

BELGIAN ROYAL METEOROLOGICAL INSTITUTE DOURBES, BELGIUM

BRITISH DEPARTMENT OF SCIENTIFIC AND INDUSTRIAL RESEARCH, RADIO RESEARCH BOARD

SIMGAPORE, BRITISH MALAYA

SLOUGH, ENGLAND

DEFENCE RESEARCH BOARD. CANADA.

CHURCHILL, CANADA

KENORA: CANADA

OTTAWA, CANADA

RESOLUTE BAY, CANADA

ST. JOHNS. NEWFOUNDLAND

UNIVERSIDAD DE CONCEPCION

CONCEPCION, CHILE

RADIO WAVE RESEARCH LABORATORIES, DIRECTORATE GENERAL OF TELECOMMUNICATIONS, MINISTRY OF COMMUNICATIONS,

TAIPEI, HSIAN, TAIWAN, REPUBLIC OF CHINA

TAIPEI (TAIWAN), CHINA

INSTITUTO GEOFISICO DE LOS ANDES COLOMBIANOS BOGOTA, COLOMBIA

CENTRAL AFRICAN INSTITUTE FOR SCIENTIFIC RESEARCH LWTRO, CONGO

CZECHOSLOVAK ACADEMY OF SCIENCES

PRUHONICE, CZECHOSLOVAKIA

DANISH NATIONAL COMMITTEE OF URSI

GODHAVN, GREENLAND

NARSSARSSUAQ, GREENLAND

GENERAL DIRECTION OF POSTS AND TELEGRAPHS, HELSINKI, FINLAND NURMI JARVI, FINLAND

THE FINNISH ACADEMY OF SCIENCES AND LETTERS SODANKYLA, FINLAND

HEINRICH HERTZ INSTITUTE, GERMAN ACADEMY OF SCIENCES,

BERLIN, GERMANY JULIUSRUH/RUGEN, GERMANY 
IONOSPHERE INSTITUTE, NATIONAL OBSERVATORY OF ATHENS ATHENS (SCARAMANGA), GREECE

ICELANDIC POST AND TELEGRAPH ADMINISTRATION

REYKJAVIK, ICELAND

INDIAN COUNCIL OF SCIENTIFIC AND INDUSTRIAL RESEARCH,

RADIO RESEARCH COMMITTEE, NEW DELHI, INDIA KODAIKANAL, INDIA (INDIA METEOROLOGICAL DEPARTMENT)

NATIONAL INSTITUTE. OF GEOPHYSICS, CITY UNIVERSITY, ROME, ITALY ROME, I TALY

MINISTRY OF POSTS AND TELECOMMUNICATIONS, RADIO RESEARCH

LABORATORIES, TOKYO, JAPAN

AKITA, JAPAN

KOKUBUNJI, TOKYO, JAPAN

WAKKANAI, JAPAN

YAMAGAWA, JAPAN

THE ROYAL NETHERLANDS METEOROLOGICAL INSTITUTE

DE BILT, NETHERLANDS

CHRISTCHURCH GEOPHYSICAL OBSERVATORY, NEW ZEALAND DEPARTMENT OF SCIENTIFIC AND INDUSTRIAL RESEARCH

GODLEY HEAD (CHRISTCHURCH), N•Z.

NORWEGIAN DEFENCE RESEARCH ESTABLISHMENT,

KJELLER PER LILLESTROM, NORWAY

TROMSO, NORWAY

MANILA OBSERVATORY, PHILIPPINES

MANILA, LUZON

INSTI TUTE OF TELECOMMUNICATION, WARSAW, POLAND

WARSAW (MIEDZESZYN), POLAND

EBRO OBSERVATORY

TORTOSA, SPAIN

RESEARCH INSTITUTE OF NATIONAL DEFENCE, STOCKHOLM, SWEDEN

KIRUNA, SWEDEN

LYCKSELE, SWEDEN

UPPSALA, SWEDEN

ROYAL BOARD OF SWEDISH TELEGRAPHS, RADIO DEPARTMENT,

STOCKHOLM, SWEDEN

LULEA, SWEDEN

POST, TELEPHONE AND TELEGRAPH ADMINISTRATION,

BERNE, SWITZERLAND

SOTTENS, SWITZERLAND 
UNITED STATES ARMY SIGNAL CORPS, UNITED STATES OF AMERICA ADAK, A.LASKA

FT. MONMOUTH, NEW JERSEY

GRAND BAHAMA I.

OKINAWA I.

THULE, GREENLAND

NATIONAL BUREAU OF STANDARDS, UNITED STATES OF AMERICA

(CENTRAL RAOIO PROPAGATION LABORATORY)

ANCHORAGE, ALASKA

BOULDER, COLORADO

FT. BELVOIR, VIRGINIA

HUANCAYO, PERU (INSTITUTO GEOFISICO DEL PERU)

MAUI, HAWAII

POLE STATION, ANTARCTICA

TALARA, PERU (INSTITUTO GEOFISICO DEL PERU) 
July 1964 - January 1962

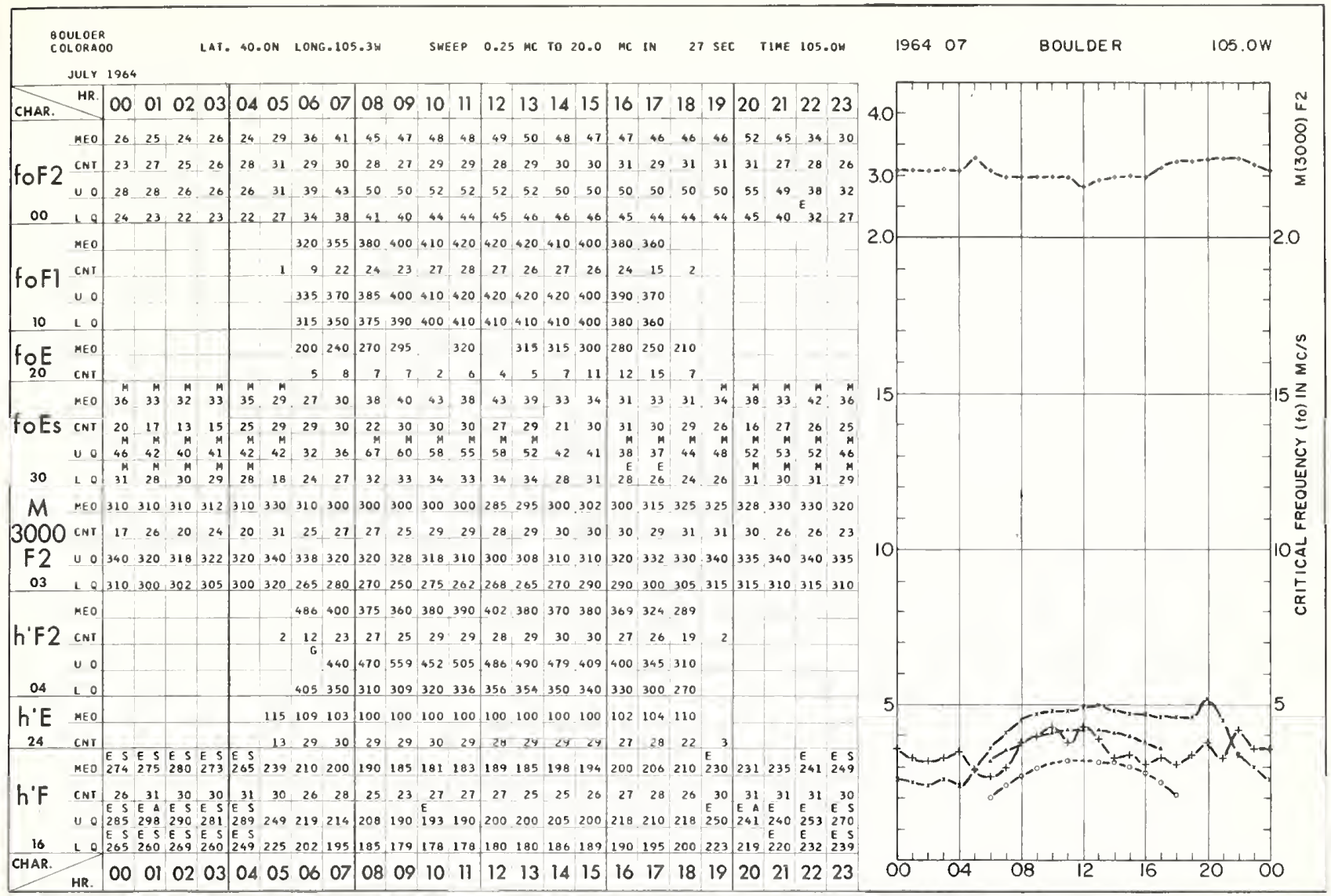

BOULOER

8OULOER
COLORAOO, U.S.A. LAT. 40.0N LONG.105.3 W SWEEP O.25 MC TO 20.0 MC IN 27 SEC TIME 105.0 H JUME 1964

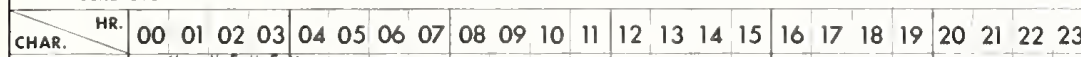

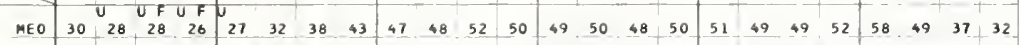

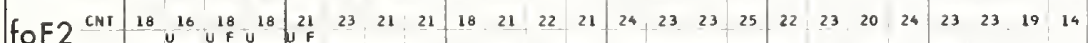

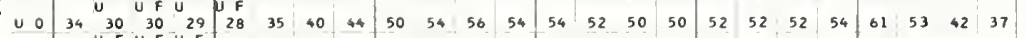

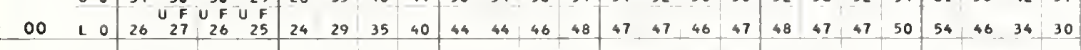$$
\text { . }
$$

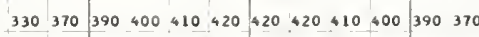$$
\begin{array}{lll|lllllllll|lll}
\hline 1 & 12 & 16 & 11 & 17 & 19 & 15 & 22 & 19 & 21 & 22 & 18 & 8 & 1
\end{array}
$$

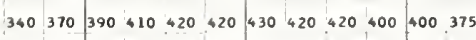

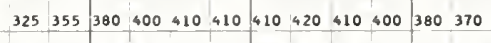

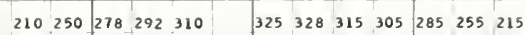$$
\text { fo }
$$$$
10 \perp 0
$$

$20 \mathrm{cmr}$

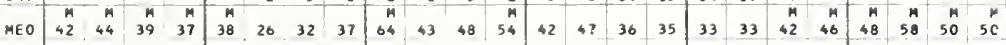

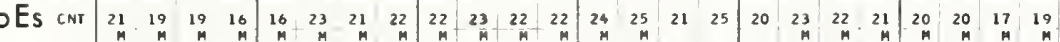

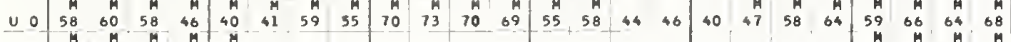

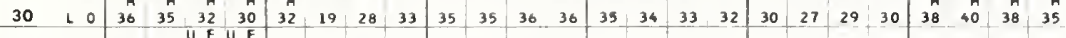

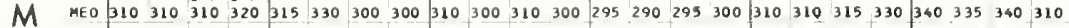
\begin{tabular}{|l|lll|l|lllll|l|l|ll|lllll|l|l|l|l|l|l}
$3000 \mathrm{CNI}$ & 13 & 7 & 8 & 11 & 12 & 21 & 21 & 20 & 18 & 21 & 22 & 21 & 24 & 22 & 22 & 25 & 21 & 22 & 19 & 23 & 21 & 21 & 13 & 10
\end{tabular}

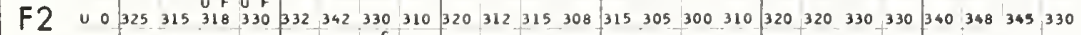

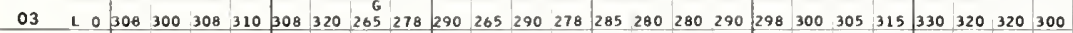

$$
\begin{array}{|l}
\hline \text { h' } \\
\hline \text { h' } \\
\hline \text { h' } \\
\hline
\end{array}
$$

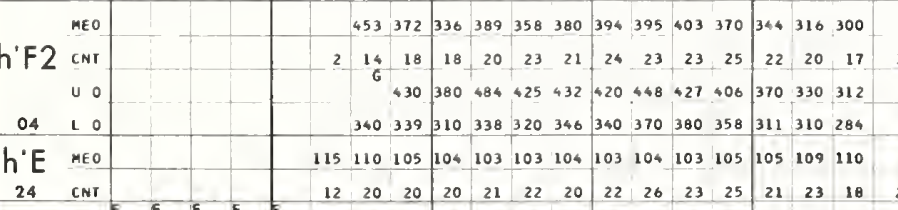

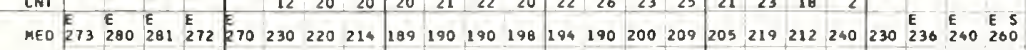

\begin{tabular}{ll|l|l|l|l|l|l|l|l|l|l|l|l|l|l|l|l|l|l|l|l|l|l|l|l|l|l} 
h'F $\quad$ CNT & 21 & 19 & 19 & 21 & 23 & 23 & 21 & 16 & 11 & 14 & 18 & 15 & 20 & 19 & 21 & 23 & 19 & 17 & 12 & 23 & 23 & 24 & 22 & 18 \\
\hline
\end{tabular}

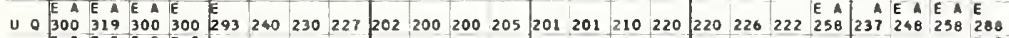

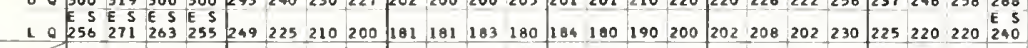

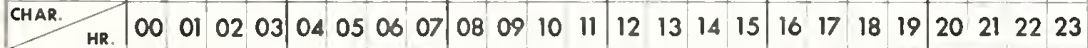

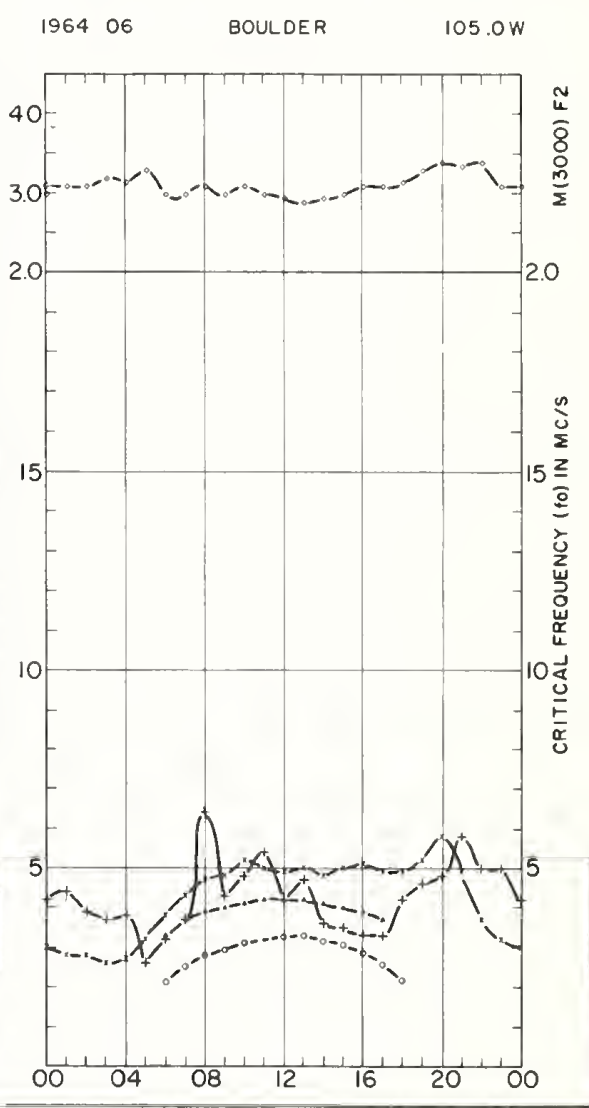




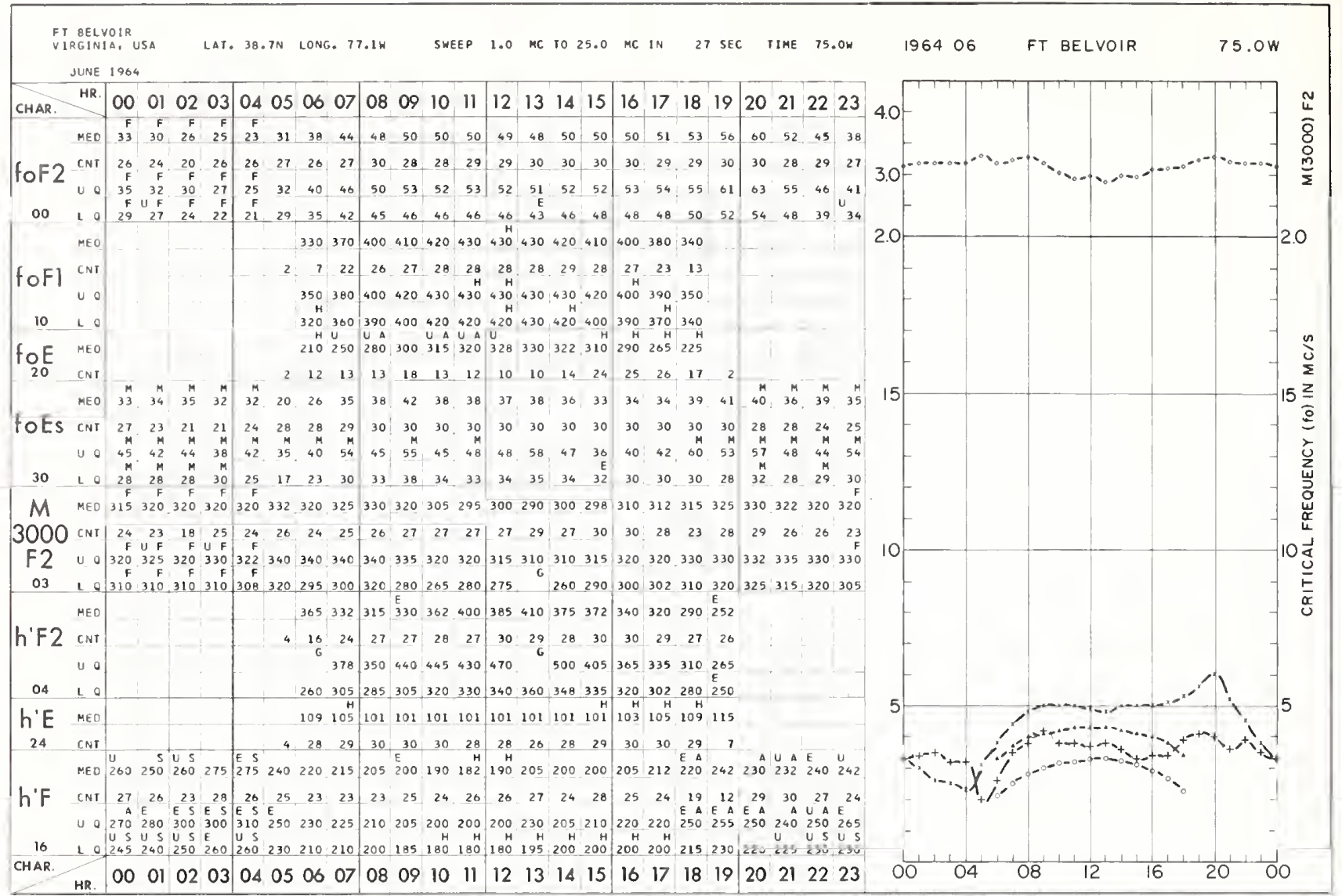

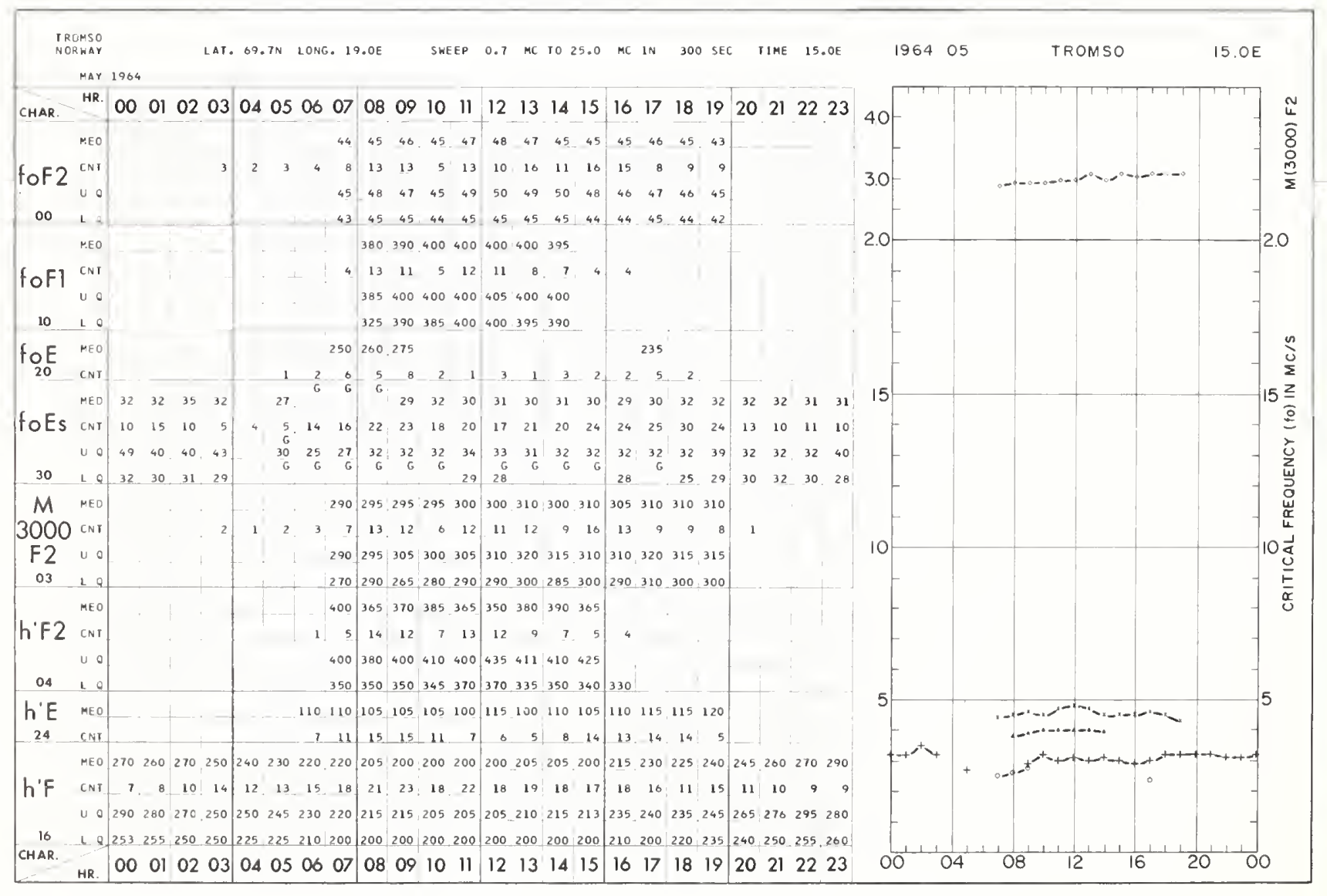


SOOANKYLA

MAY 1964

LAT. $67.4 \mathrm{~N}$ LONG. $26.6 \mathrm{E}$

SWEEP 1.4 MC TO 22.0 MC IN 480 SEC TIME 30.0 .

196405

SODANKYLA

$30.0 E$

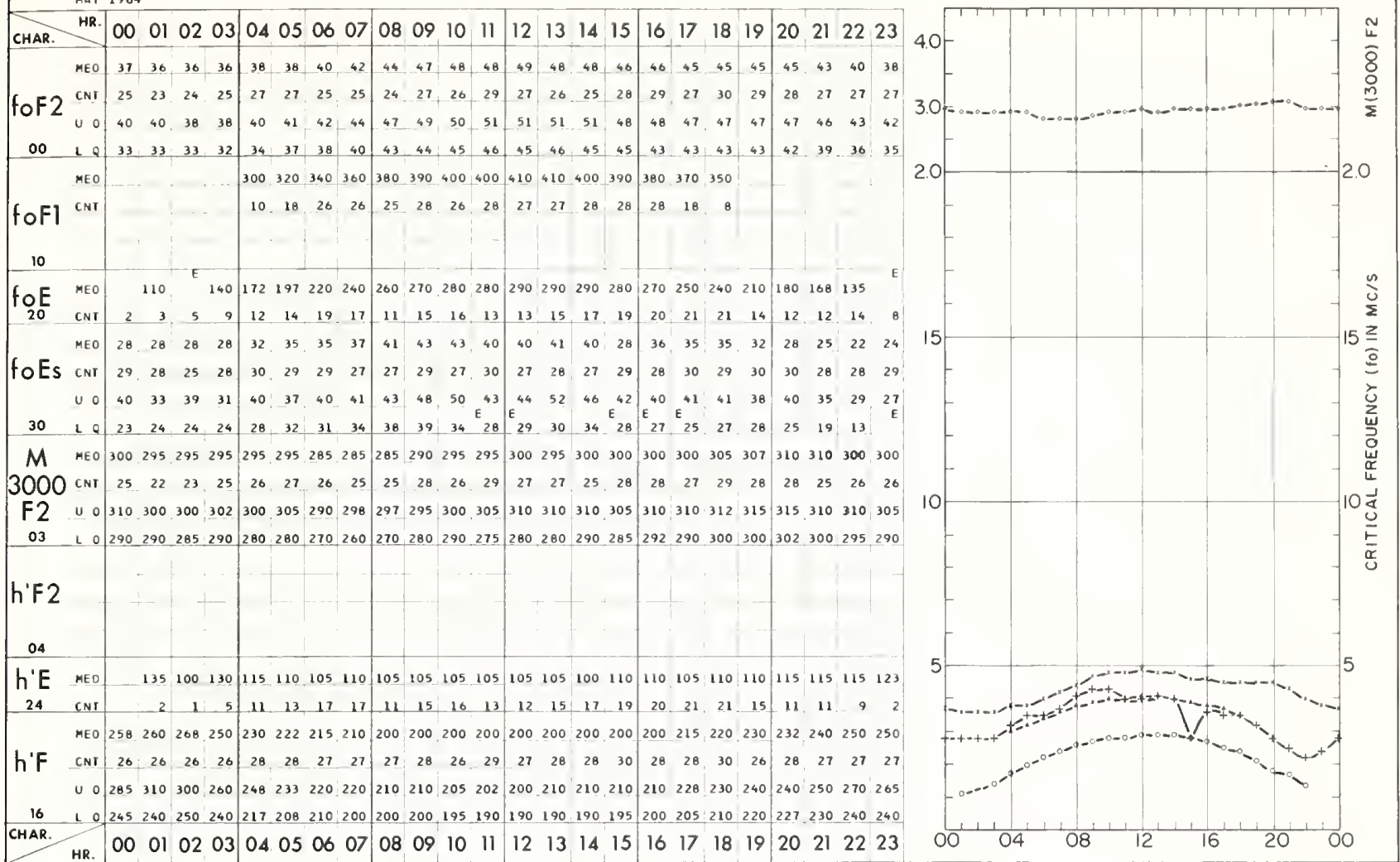

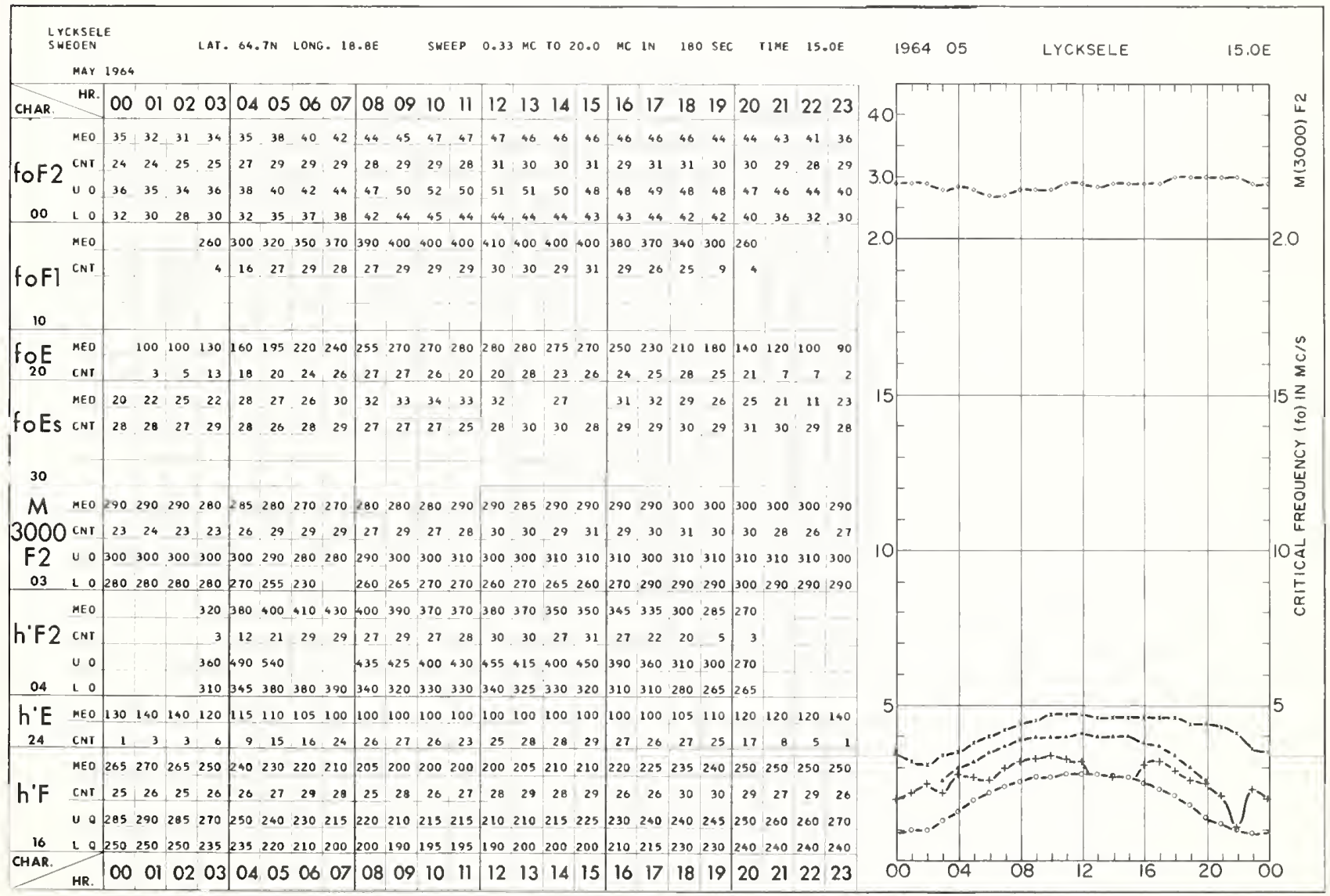




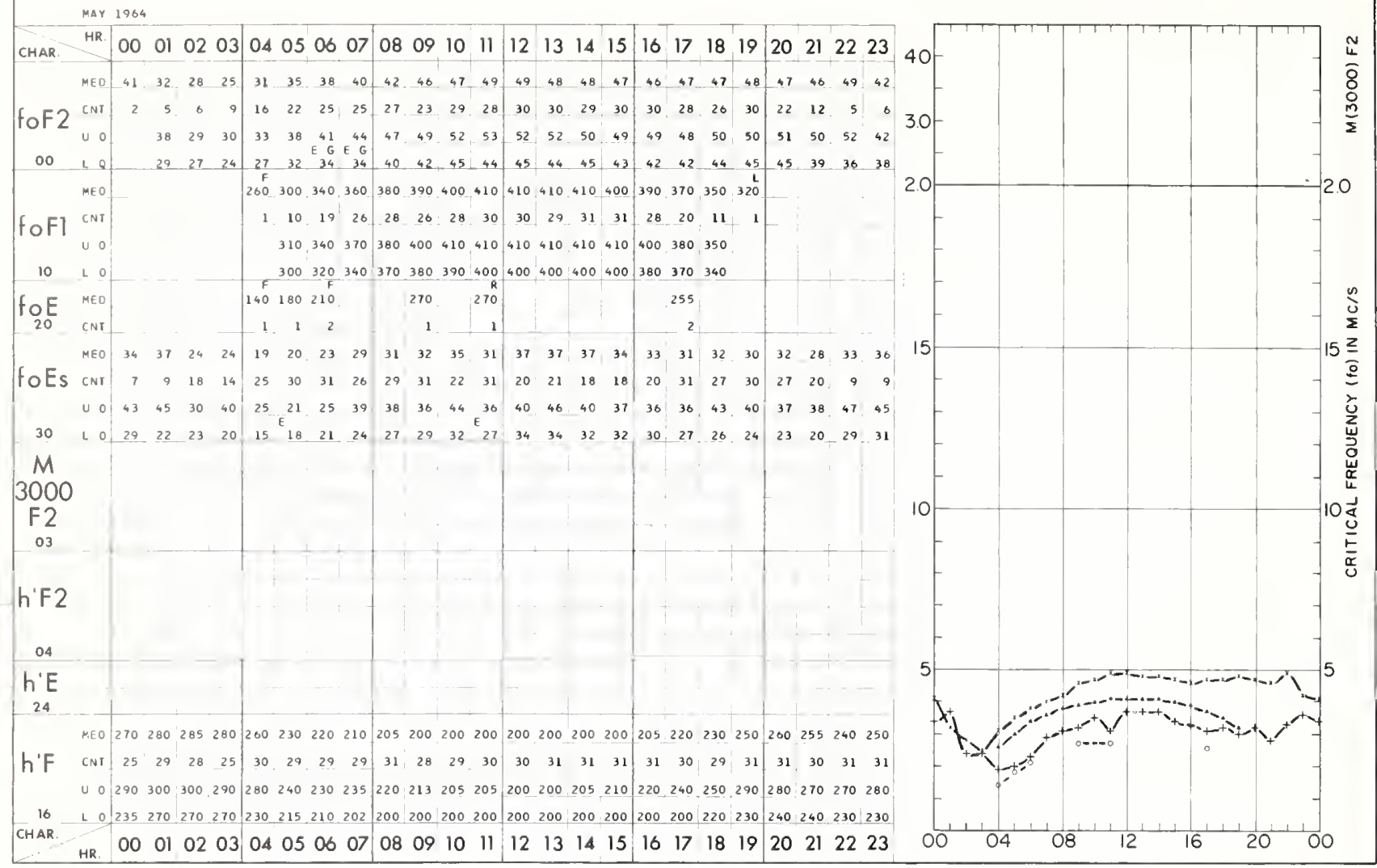

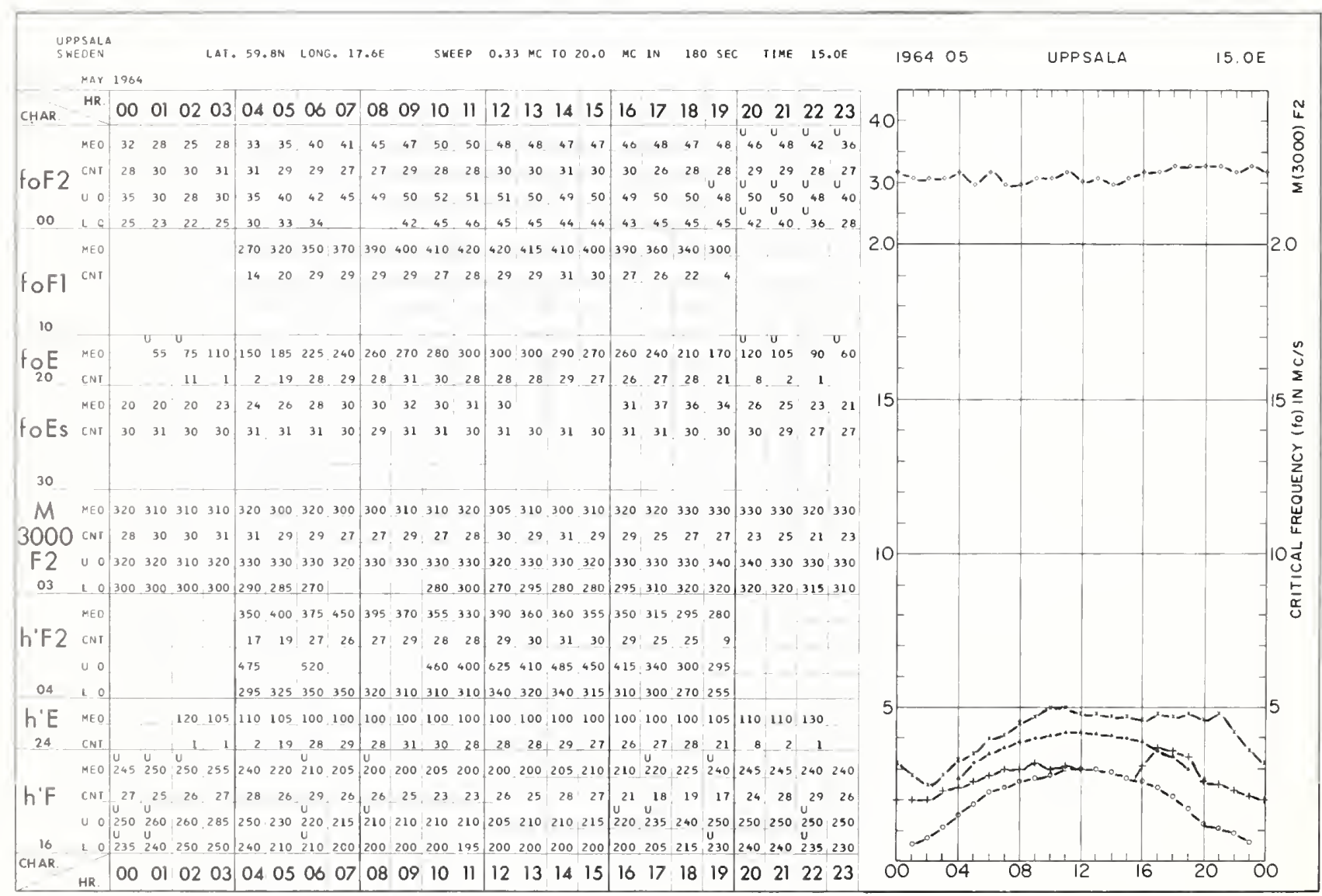


MAY 1964

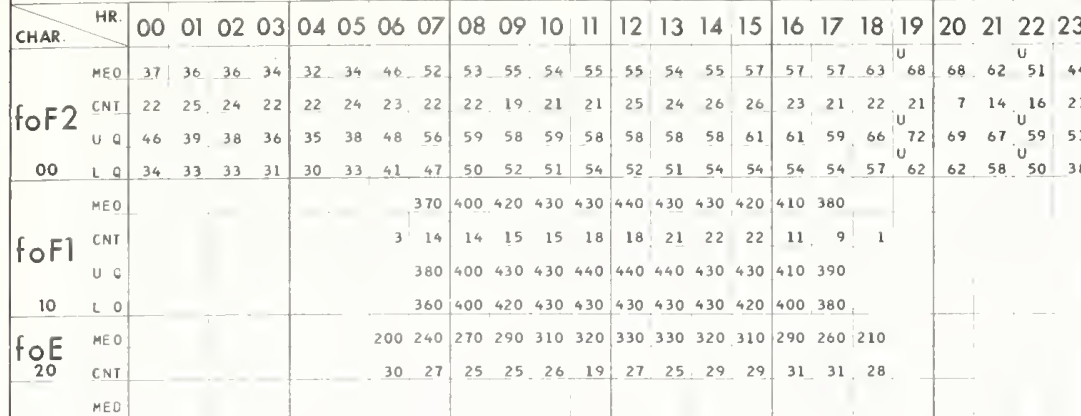

\begin{tabular}{|l|llll|llll|lllll|lllll|llll|llll}
\hline foES CNT & 30 & 30 & 30 & 30 & 30 & 30 & 30 & 30 & 30 & 30 & 30 & 29 & 31 & 30 & 30 & 29 & 30 & 31 & 30 & 30 & 31 & 30 & 30 & 30
\end{tabular} 30

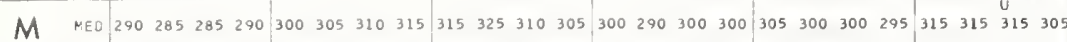

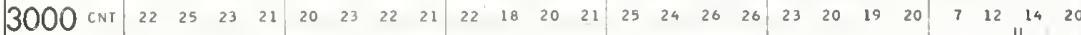

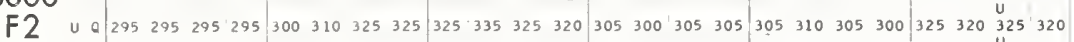

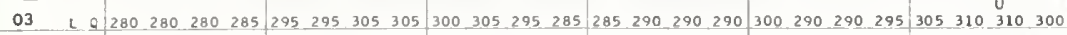

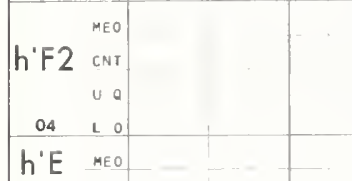
$315305 \quad 300 \quad 315 \quad 340 \quad 345 \quad 375 \quad 350 \quad 330 \quad 310 \quad 340$ \begin{tabular}{ll|llll|lllll|llll}
3 & 14 & 14 & 16 & 18 & 20 & 20 & 24 & 25 & 26 & 13 & 9 & 1
\end{tabular}

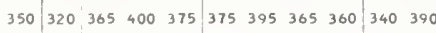
\begin{tabular}{ll|l|l|l|l|l|l|l|l|l}
290 & 290 & 285 & 300 & 305 & 335 & 345,340 & 320 & 310 & 310
\end{tabular}

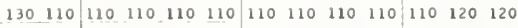
$\begin{array}{llllllllllllll}30 & 30 & 30 & 30 & 30 & 30 & 31 & 31 & 30 & 30 & 31 & 31 & 30\end{array}$

\begin{tabular}{l|llll|llll|l|l|l|l|l|l|l|l|l|l|l|l|l|l|l|l|} 
MEO & 280 & 300 & 300 & 290 & 280 & 260 & 240 & 230 & 225 & 210 & 200 & 210 & 205 & 210 & 210 & 240 & 255 & 240 & 250 & 270 & 250 & 240 & 240 & 250
\end{tabular} \begin{tabular}{|llllll|llll|lllll|llll|llll|llll} 
h $^{\prime} F$ & CNT & 24 & 25 & 25 & 25 & 22 & 23 & 22 & 17 & 17 & 16 & 14 & 17 & 18 & 20 & 15 & 8 & 8 & 6 & 9 & 20 & 19 & 23 & 21 & 24
\end{tabular}

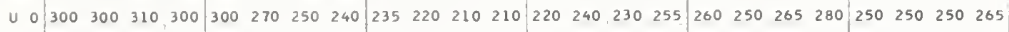

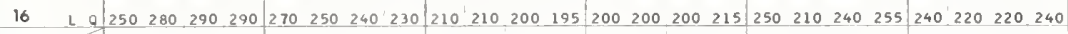
\begin{tabular}{l|llllllll|llll|llllllllllll} 
CHAR. & 00 & 01 & 02 & 03 & 04 & 05 & 06 & 07 & 08 & 09 & 10 & 11 & 12 & 13 & 14 & 15 & 16 & 17 & 18 & 19 & 20 & 21 & 22 & 23
\end{tabular}

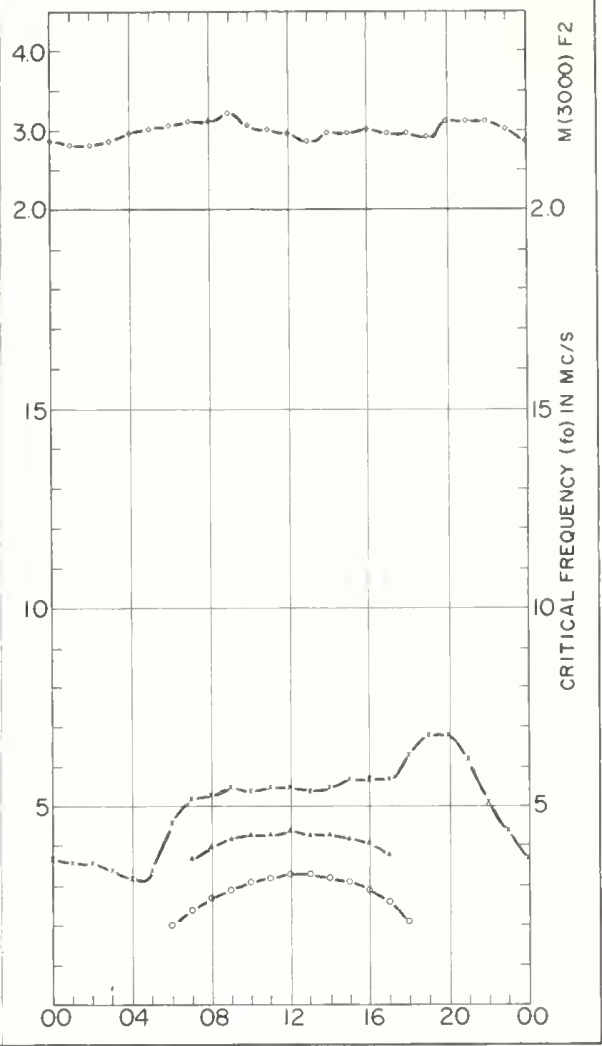

GRAND BAHAMA 1. LAT. 26.6N LONG. 78.2H SHEEP 1.0 MC TO 25.0 MC IN 27 SEC TIME $75.0 \mathrm{H}$ 1964 O5 GRAND BAHAMA I. 75.0 W \begin{tabular}{l|l|llll|llll|llll|llll|llllllll}
\hline CHAR. & HR & 00 & 01 & 02 & 03 & 04 & 05 & 06 & 07 & 08 & 09 & 10 & 11 & 12 & 13 & 14 & 15 & 16 & 17 & 18 & 19 & 20 & 21 & 22 & 23
\end{tabular} \begin{tabular}{l|llll|llll|llllllllllll|llll} 
R.EO & 39 & 37 & 35 & 32 & 31 & 30 & 42 & 52 & 53 & 54 & 53 & 55 & 59 & 63 & 66 & 68 & 68 & 68 & 72 & 71 & 60 & 46 & 40 & 38
\end{tabular}

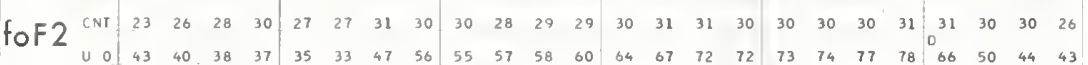
\begin{tabular}{llllll|llll|lllll|llllllll|lllll}
00 & $\mathrm{~L}$ & 9 & 33 & 33 & 32 & 30 & 29 & 27 & 38 & 47 & 47 & 49 & 50 & 51 & 54 & 58 & 59 & 62 & 60 & 57 & 63 & 63 & 53 & 37 & 32 & 32
\end{tabular} MEO :

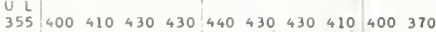
foFl $\begin{array}{cc}\text { CNT } \\ \text { U } & 0\end{array}$ \begin{tabular}{ll|llll|llll|ll}
1 & 12 & 20 & 20 & 26 & 25 & 24 & 28 & 30 & 28 & 28 & 20
\end{tabular}

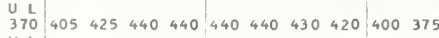

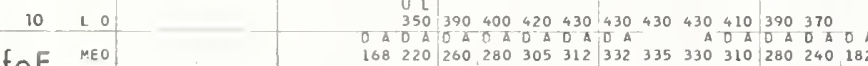

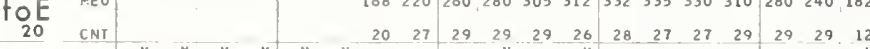

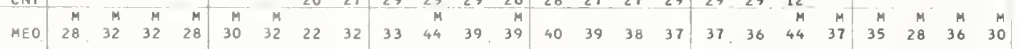

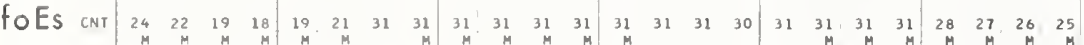

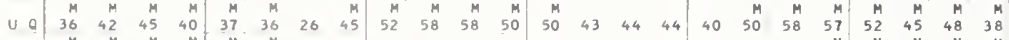

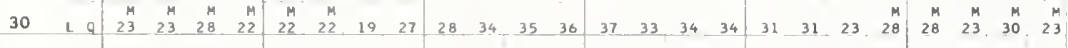
\begin{tabular}{ll|llll|lllll|llll|llll|lllllllll}
$M$ & MEO & 300 & 300 & 302 & 310 & 310 & 315 & 335 & 335 & 330 & 310 & 300 & 295 & 290 & 300 & 300 & 305 & 315 & 315 & 320 & 335 & 328 & 315 & 300 & 300
\end{tabular} \begin{tabular}{|l|llll|llll|lllll|llll|llll|lll|}
$3000 \mathrm{CNT}$ & 22 & 26 & 28 & 30 & 25 & 27 & 31 & 30 & 28 & 27 & 29 & 29 & 30 & 31 & 31 & 30 & 30 & 29 & 30 & 31 & 30 & 29 & 30 & 26
\end{tabular}

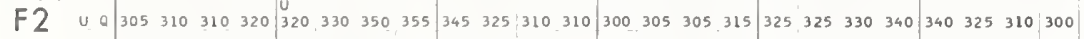

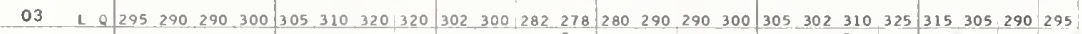

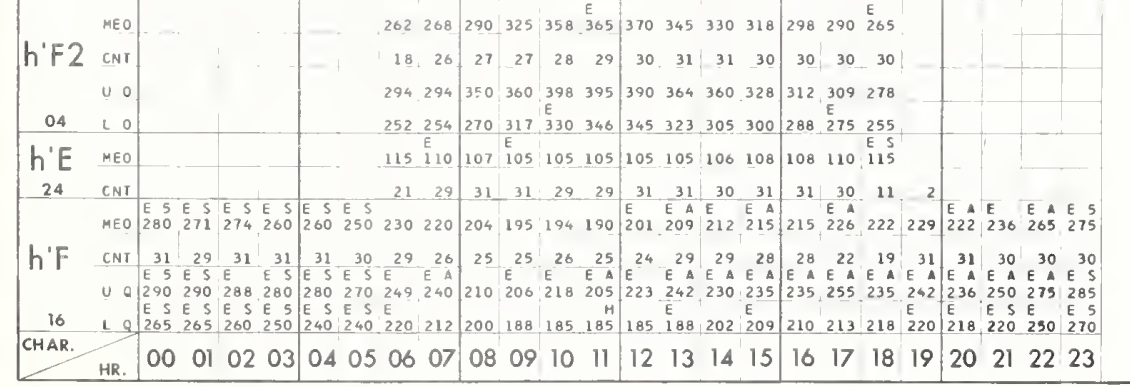

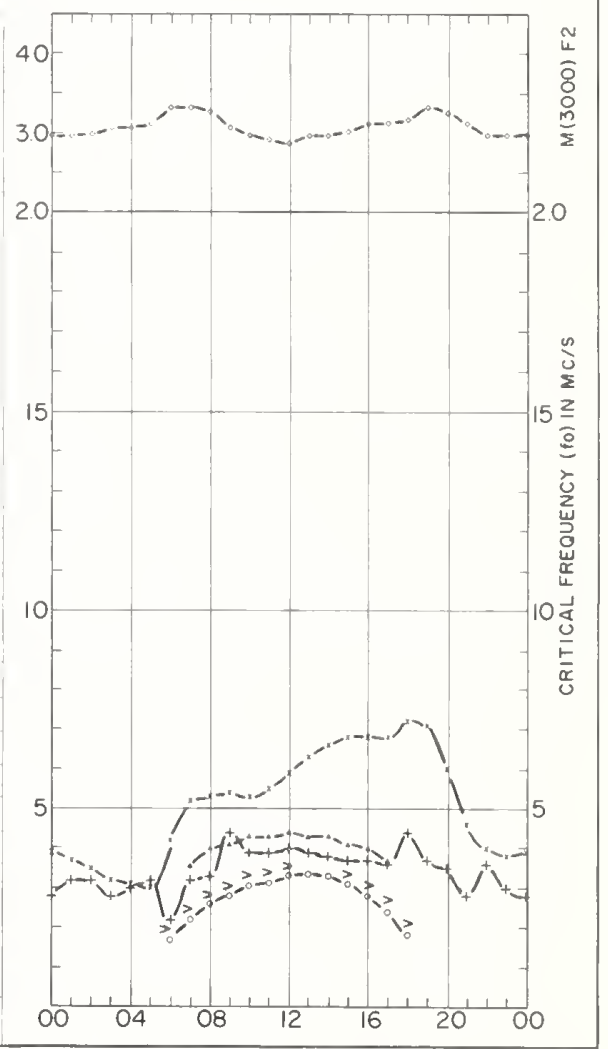



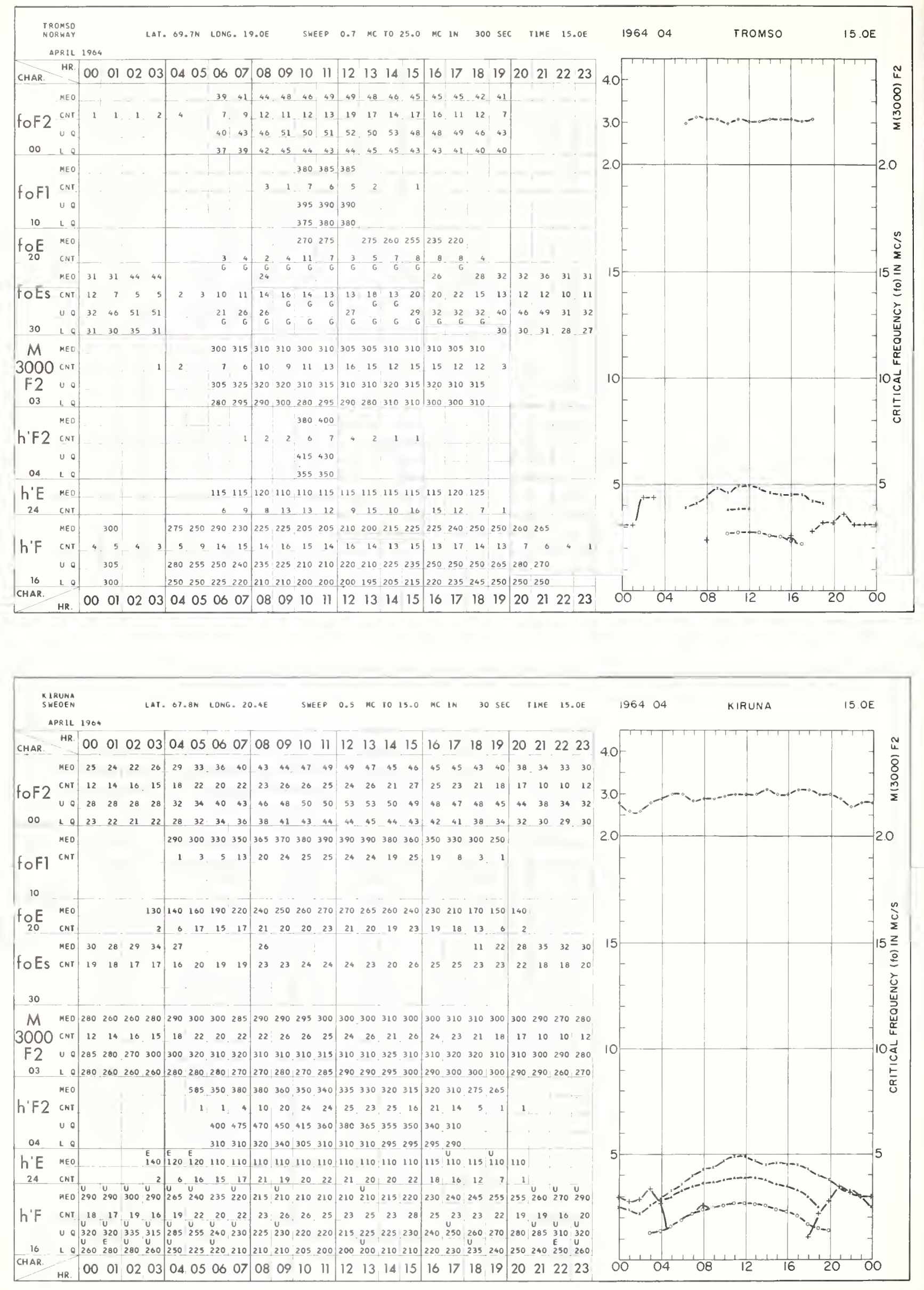
APRIL 1964

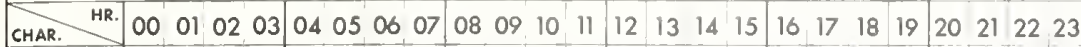

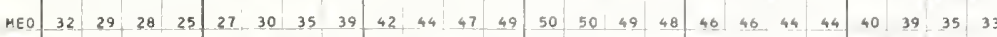

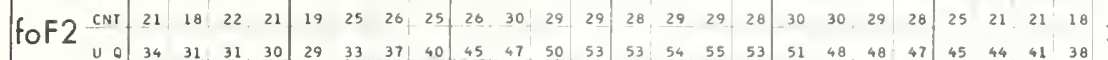

\begin{tabular}{lll|llll|l|l|l|l|l|l|l|l|l|l|l|l|l|l|l|l|l|l|l|l|}
00 & 1 & 9 & 29 & 26 & 23 & 22 & 25 & 28 & 33 & 37 & 38 & 41 & 43 & 45 & 47 & 46 & 46 & 45 & 45 & 44 & 42 & 39 & 36 & 34 & 31 & 31 \\
\hline
\end{tabular}

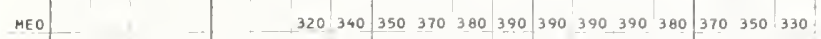

foFl \begin{tabular}{ll|llll|llll|llll}
4 & 11 & 21 & 27 & 29 & 27 & 28 & 27 & 27 & 18 & 13 & 5 & 1
\end{tabular} \begin{tabular}{ll|lll|l|l|l|l|l|l|l|l|l|l}
$330 \quad 350$ & $360 \quad 380$ & 390 & 400 & 400 & 400 & 400 & 390 & $370 \quad 350$
\end{tabular}

$10 \mathrm{~L} 0$ \begin{tabular}{llllll|l}
290,340 & $340,360 \quad 380,380$ & $390 \quad 390 \quad 380,380$ & $360 \quad 340$
\end{tabular}

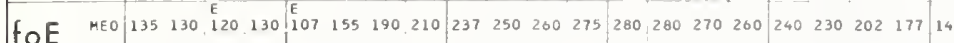

\begin{tabular}{llllll|llll|llll|llll|llllllll}
20 & $0 N T$ & 1 & 1 & 2 & 7 & 8 & 14 & 22 & 20 & 20 & 23 & 23 & 26 & 24 & 23 & 26 & 24 & 25 & 26 & 24 & 16 & 11 & 15 & 4
\end{tabular} \begin{tabular}{l|llll|lllllllll|lllll|llllllll} 
MEO & 24 & 24 & 25 & 22 & 23 & 23 & 24 & 29 & 33 & 37 & 38 & 37 & 40 & 29 & 27 & 37 & 24 & 23 & 29 & 24 & 23 & 24 & 23 & 24
\end{tabular} \begin{tabular}{|l|llll|lllll|llll|llll|llllllll} 
foES CNT & 30 & 26 & 27 & 28 & 23 & 24 & 25 & 27 & 27 & 29 & 29 & 29 & 28 & 29 & 30 & 29 & 30 & 30 & 30 & 28 & 29 & 27 & 26 & 28
\end{tabular} \begin{tabular}{llllll|lllllllll|llll|llllllllll}
\hline & 0 & 36 & 37 & 29 & 28 & 28 & 28 & 30 & 35 & 38 & 40 & 44 & 41 & 41 & 41 & 40 & 41 & 35 & 32 & 34 & 31 & 30 & 30 & 27 & 41
\end{tabular}

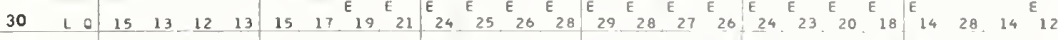

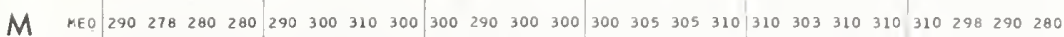

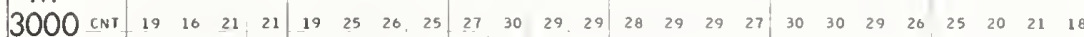

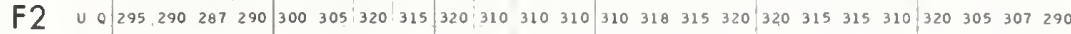

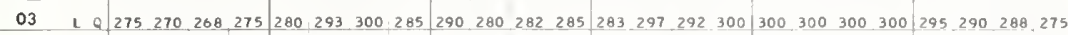

\section{h'F2}

h'.

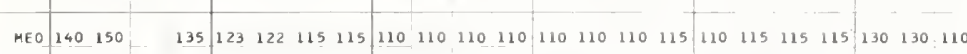

\begin{tabular}{llllllllllllll|lllllllll|llll}
24 & CNT & 1 & 1 & & 3 & 4 & 14 & 21 & 20 & 20 & 23 & 21 & 26 & 25 & 23 & 26 & 24 & 24 & 26 & 23 & 17 & 11 & 3 & 1
\end{tabular}

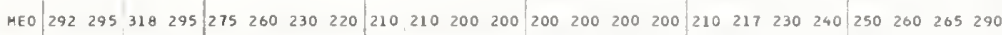

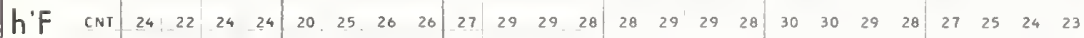

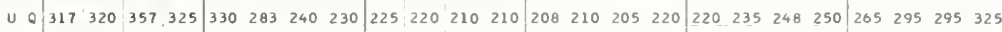

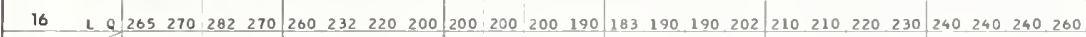
\begin{tabular}{llllll|llll|llll|llll|llllllll} 
CHAR. & 00 & 01 & 02 & 03 & 04 & 05 & 06 & 07 & 08 & 09 & 10 & 11 & 12 & 13 & 14 & 15 & 16 & 17 & 18 & 19 & 20 & 21 & 22 & 23
\end{tabular}
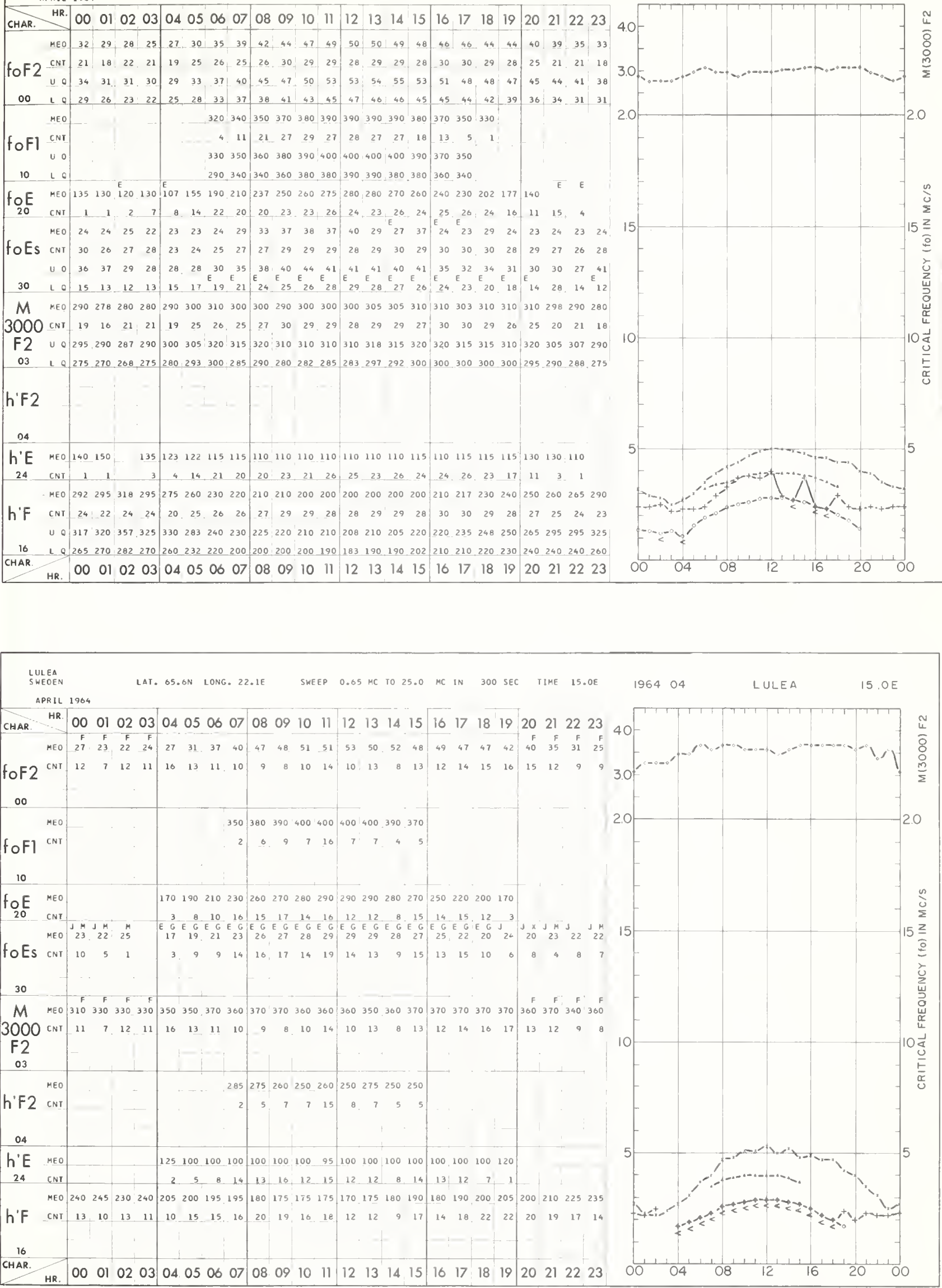

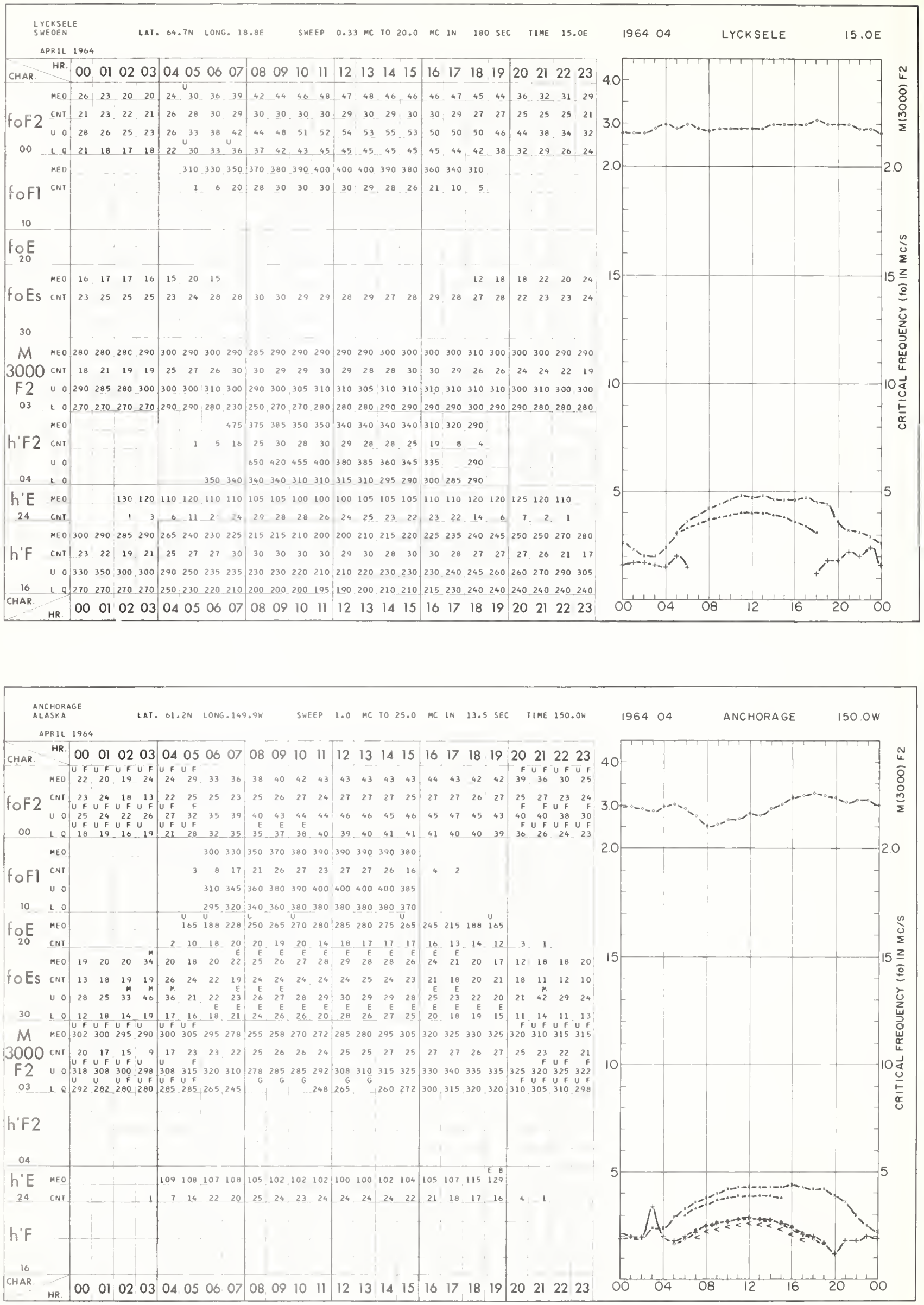
NURMI JARVI

APRIL 1964

LAT. 60.5N LONG. 24.6

SWEEP 1.0 MC TO 25.0 MC IN

60 SEC TIKE 30.0

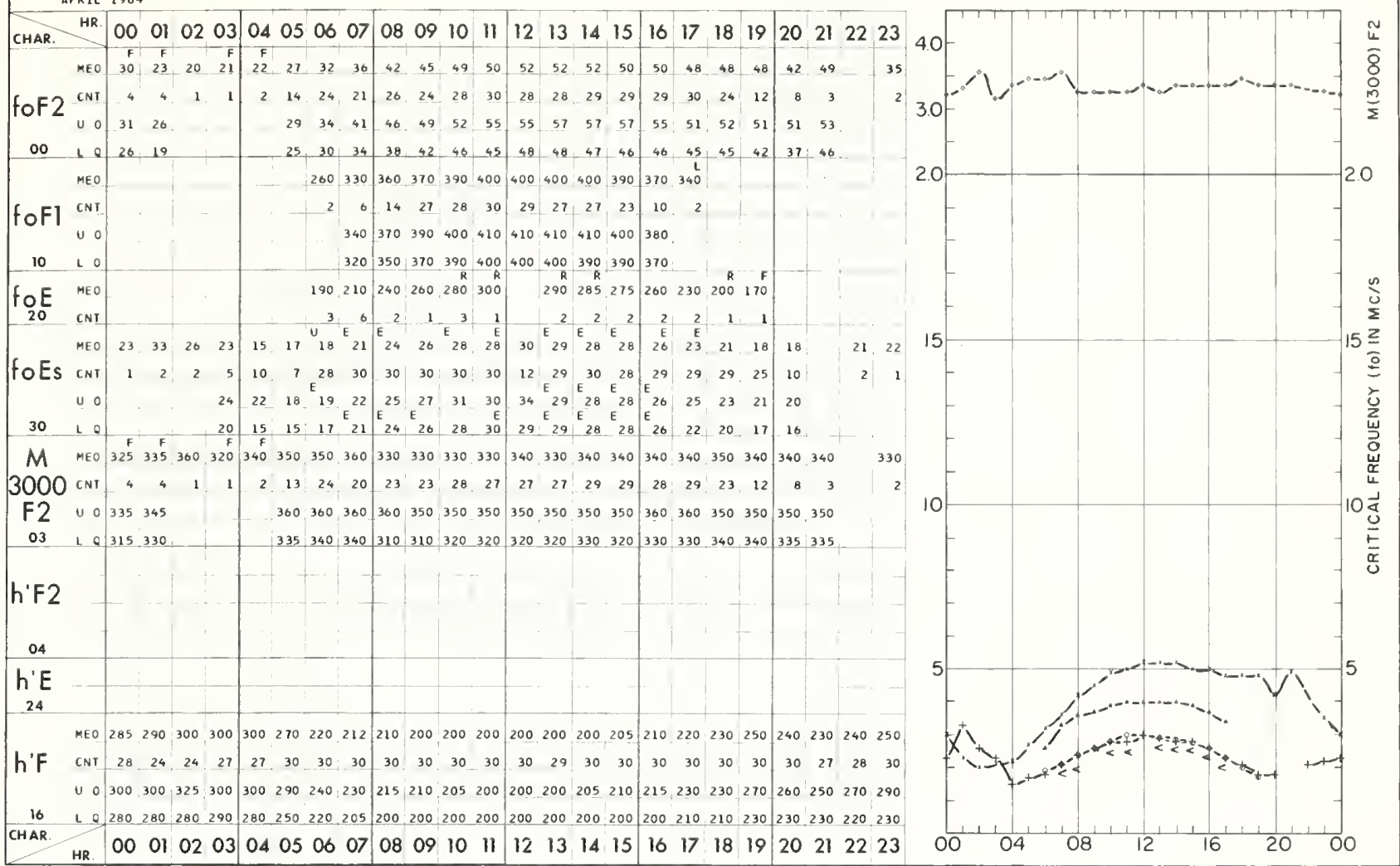

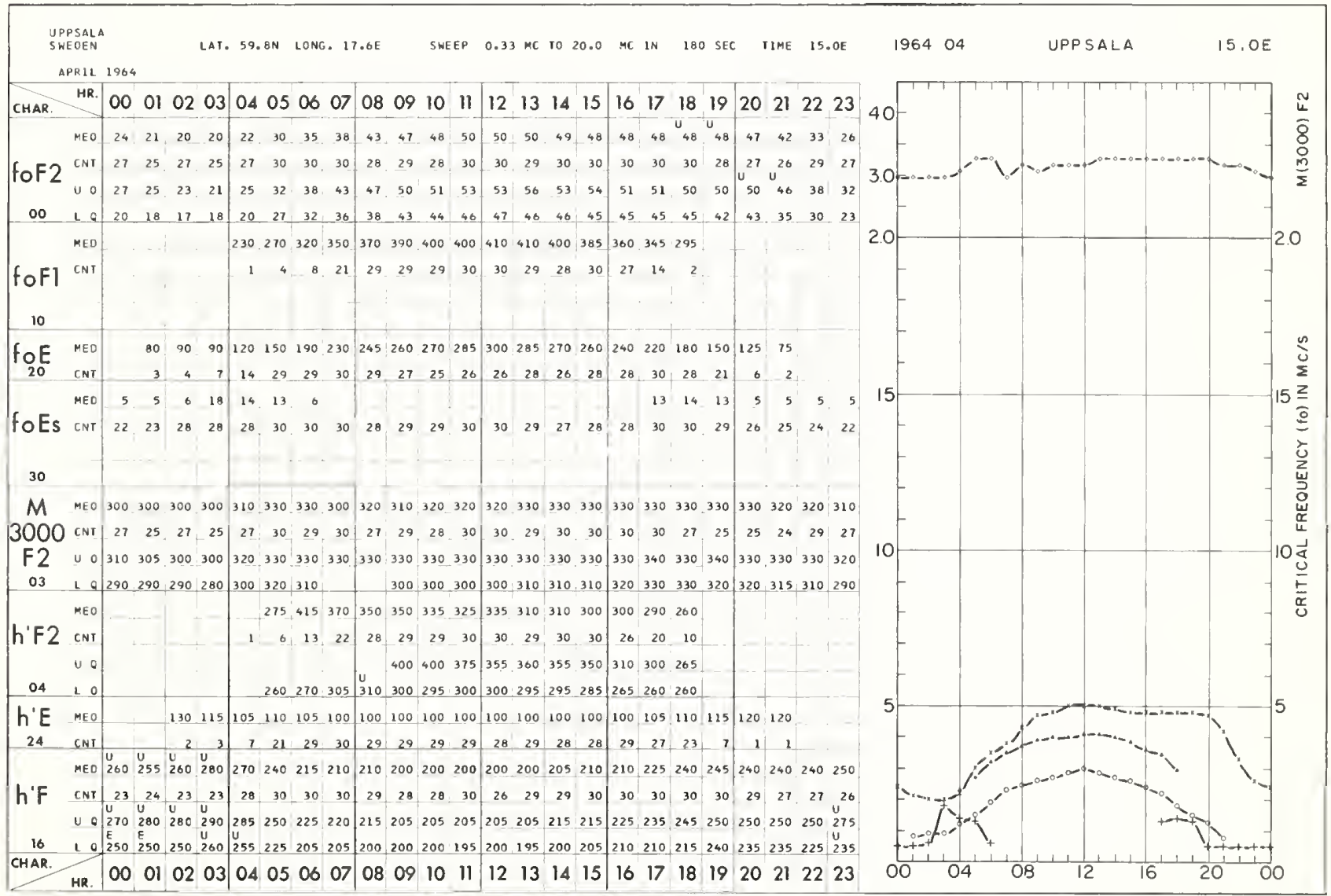




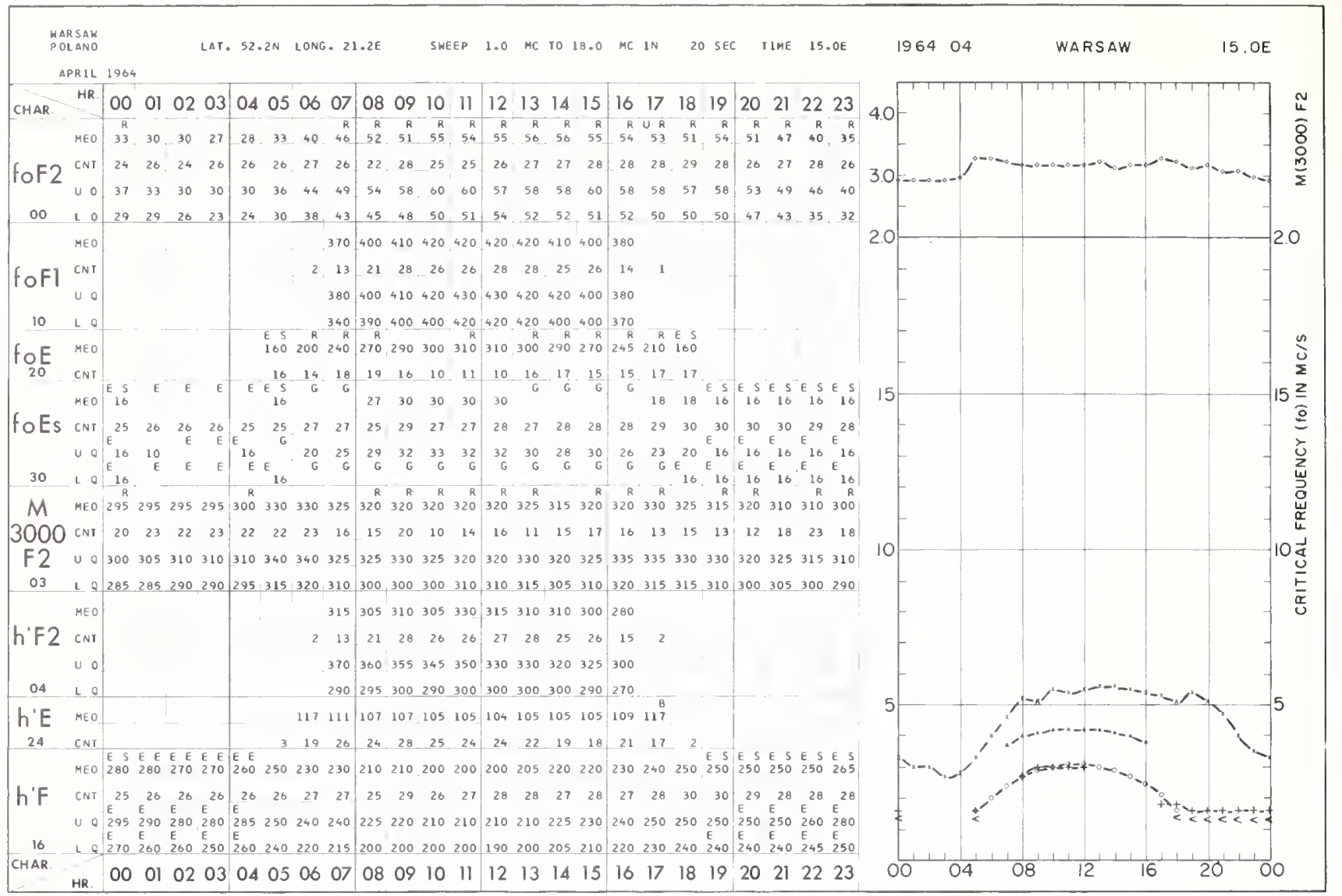

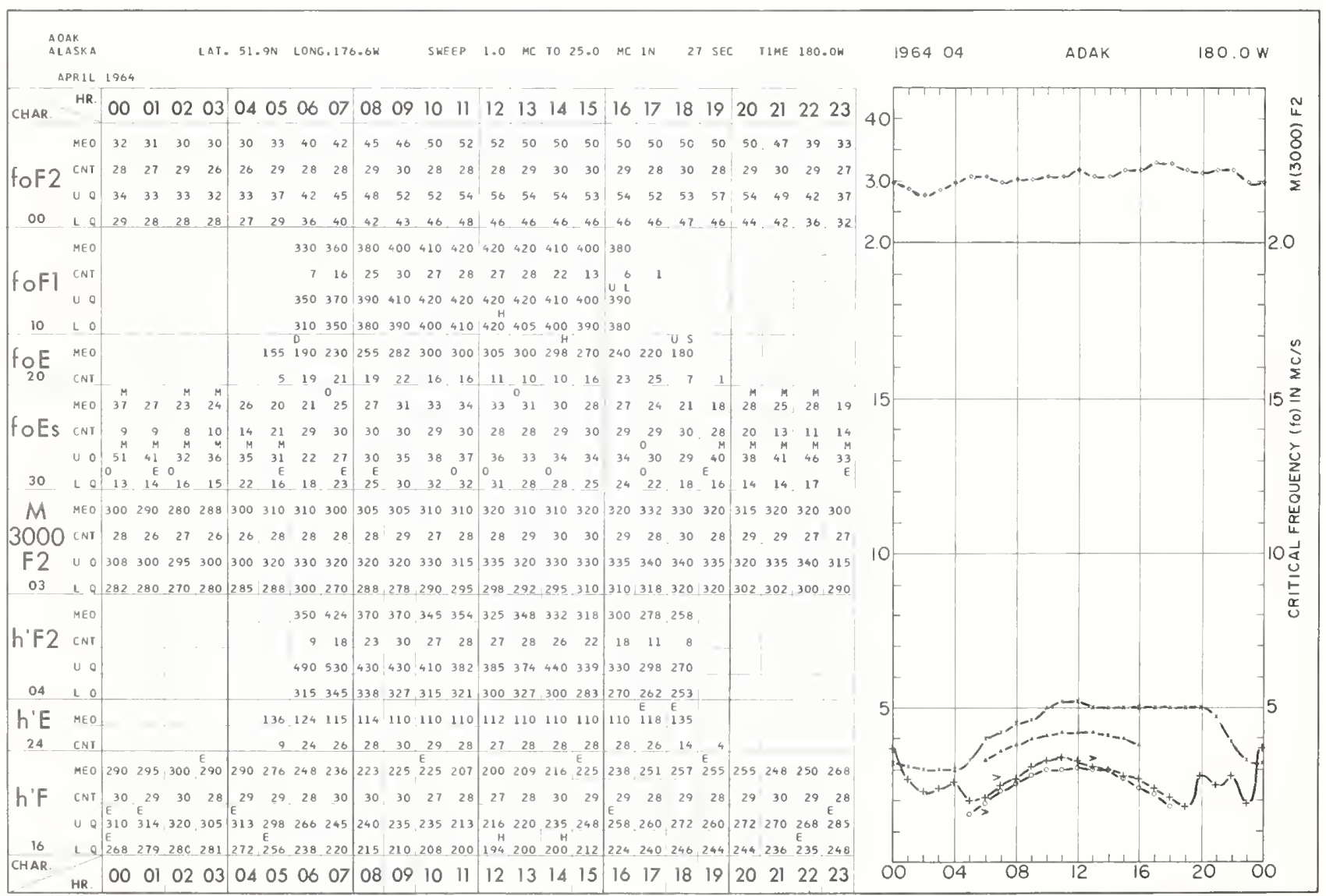




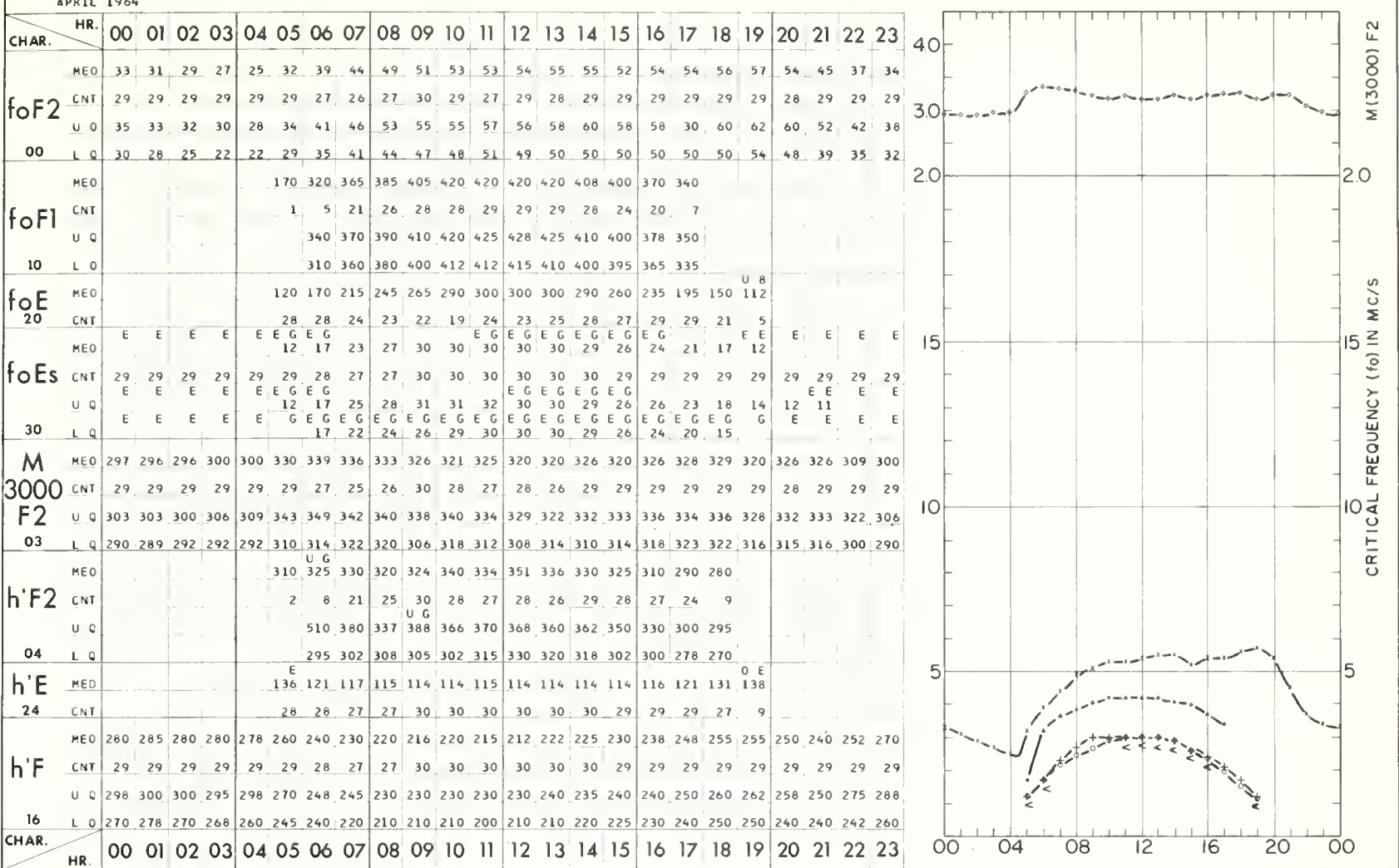

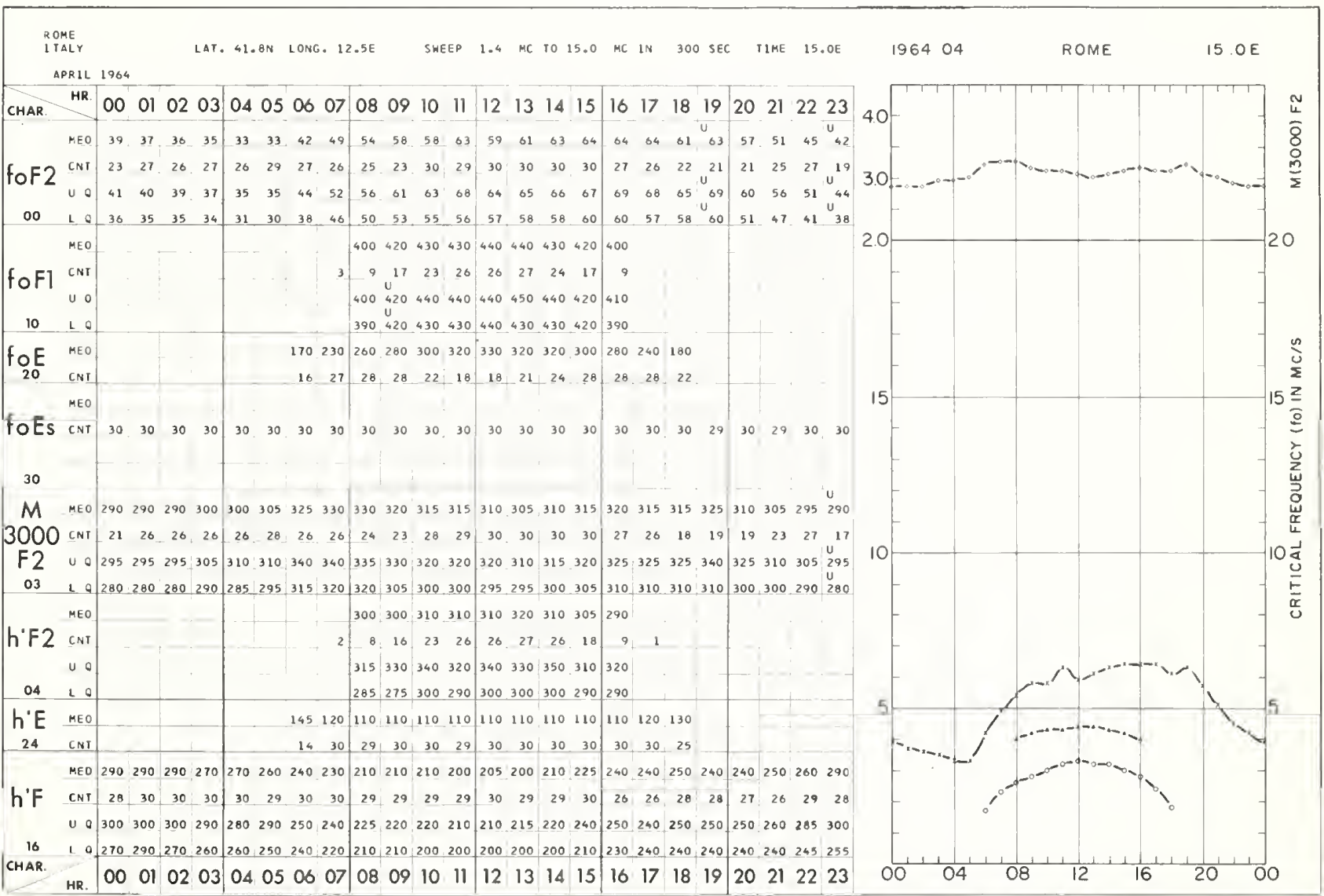


GRANO BAHAMA 1.

LAT. 26.6N LONG. 78.2W SHEEP 1.0 MC TO 25.0 MC IN 27 SEC TIME $75.0 \mathrm{~W}$

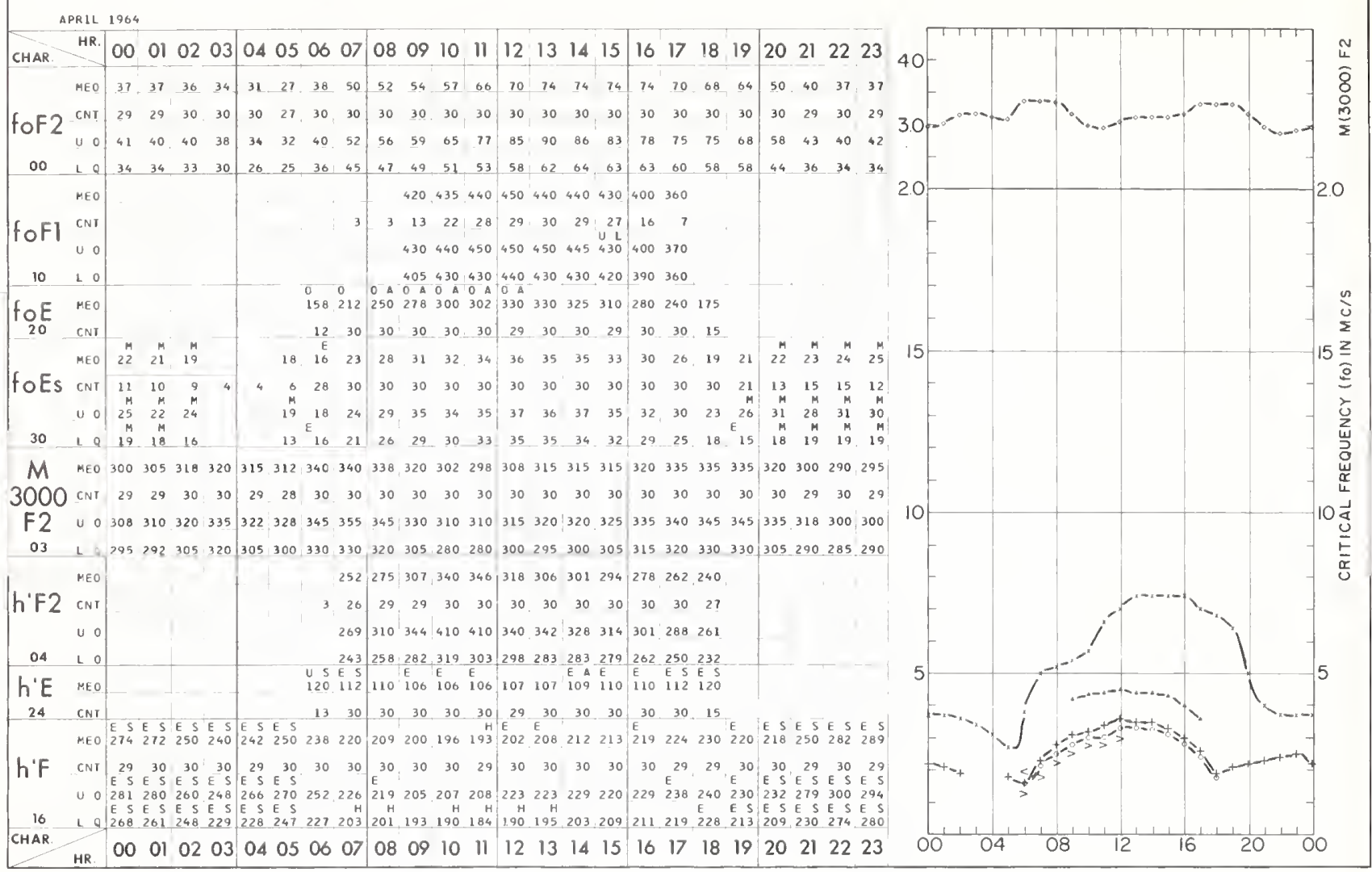

OKINAWA 1 .

LAT. 26.3N LONG.127.8E SWEEP 1.0 MC TO 25.0 MC IN 27 SEC TIME 135.0 E

196404 OKINAWA I

$135 . \mathrm{OE}$

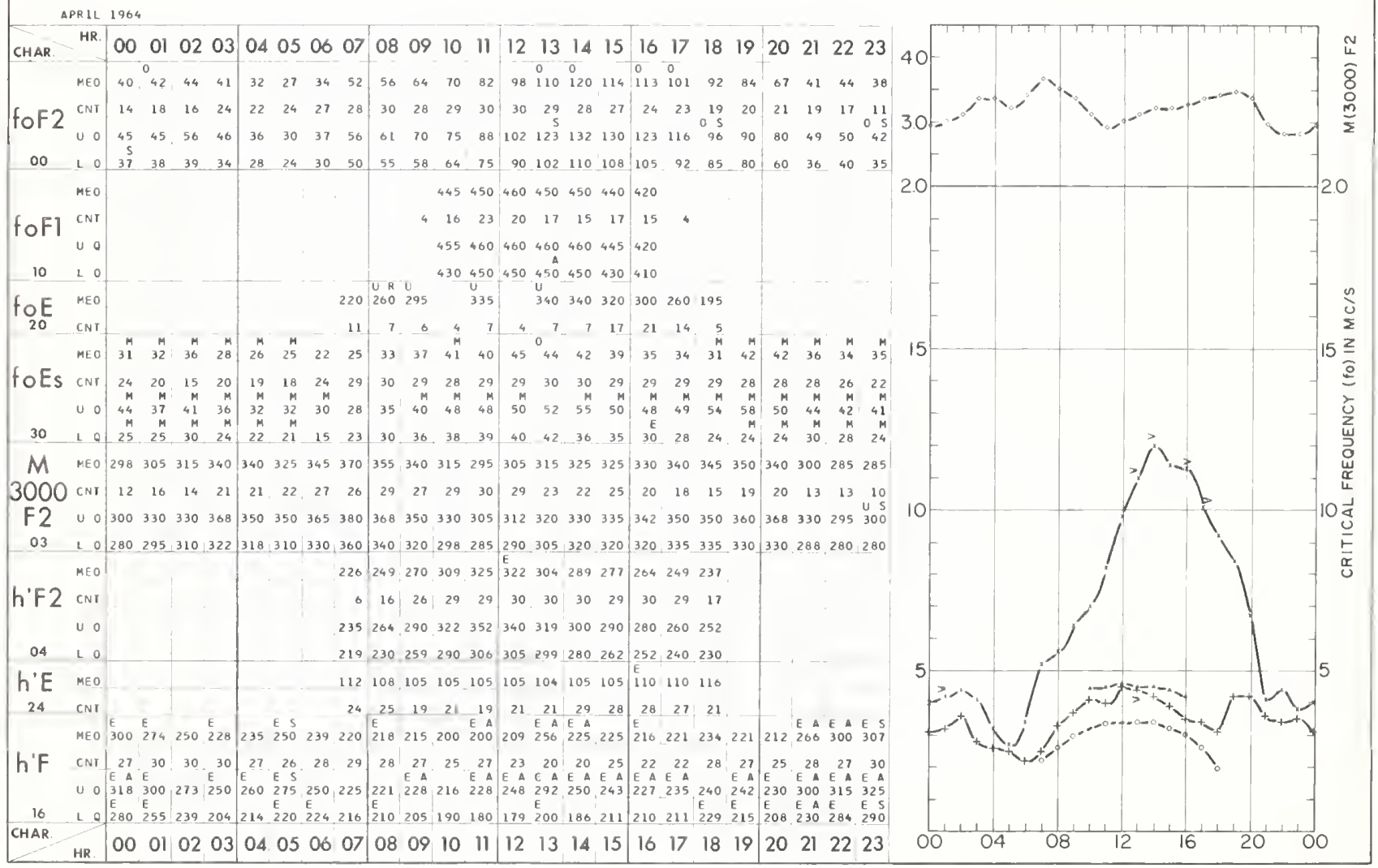


TALPEIITALWAN]

CHINA

LAT. 25.ON LONG.121.5E

SWEEP 1.0 MC TO 25.0 MC IN 27 SEC IIME 120.0 E

196404

TAIPEI

$120.0 E$

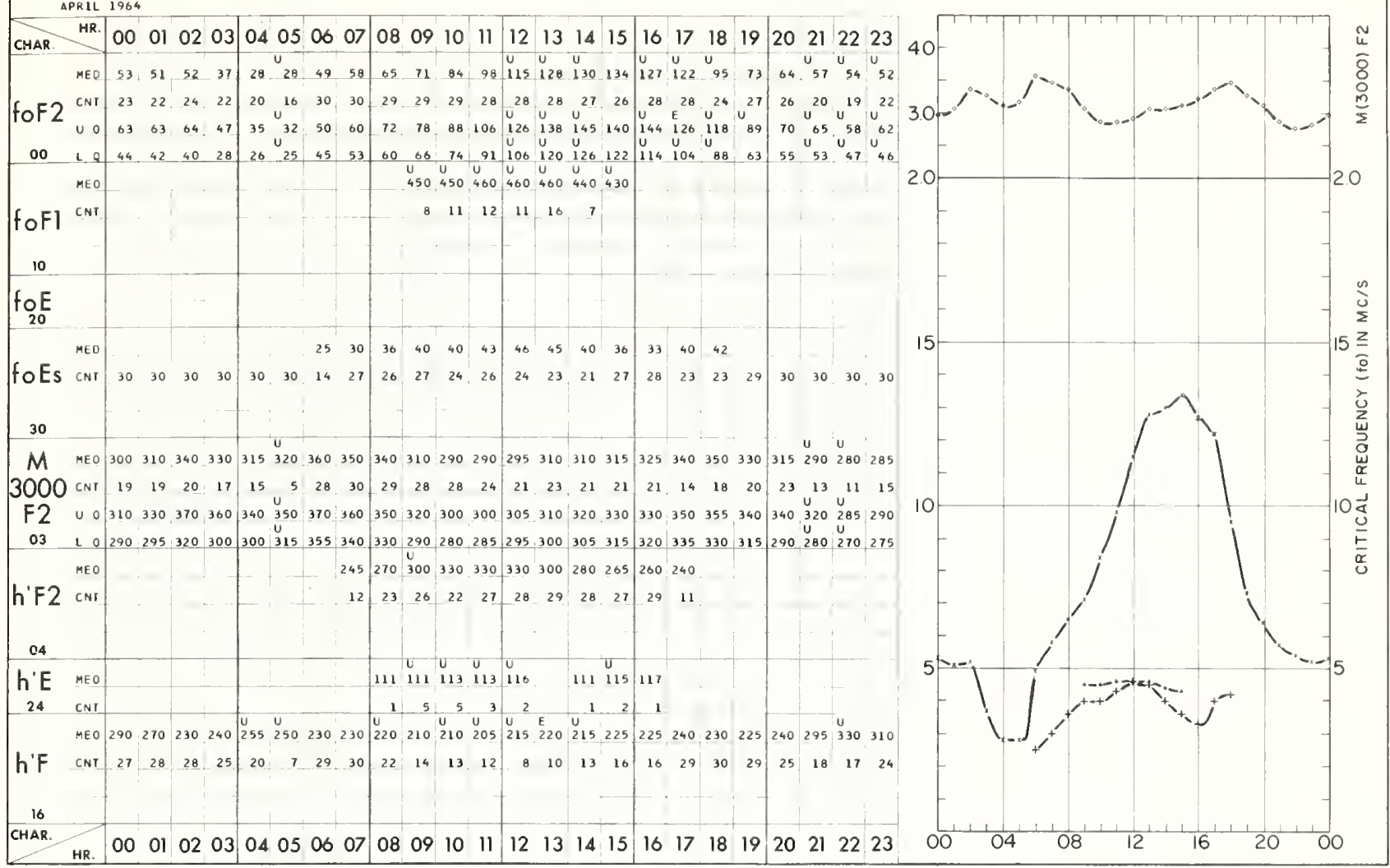

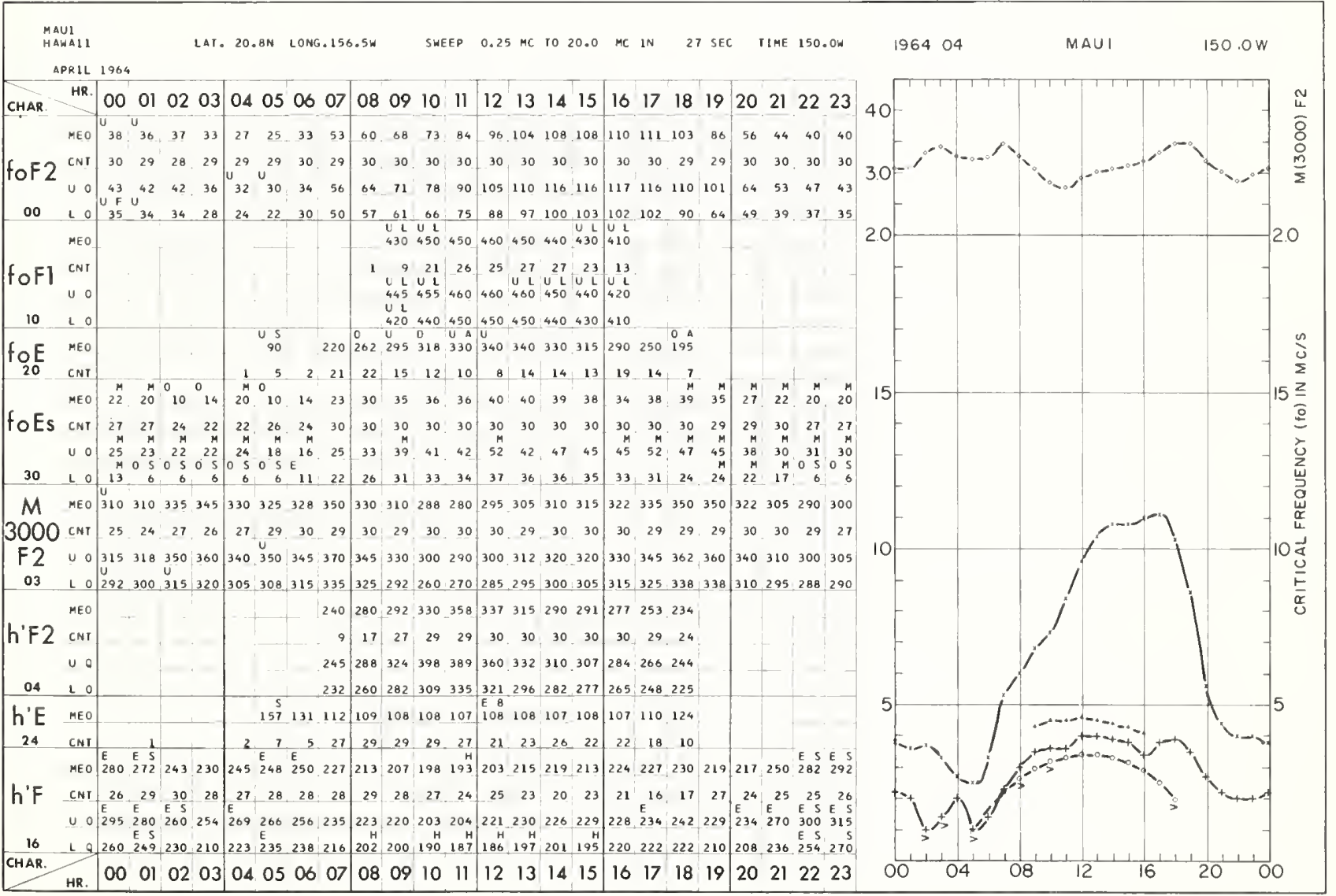



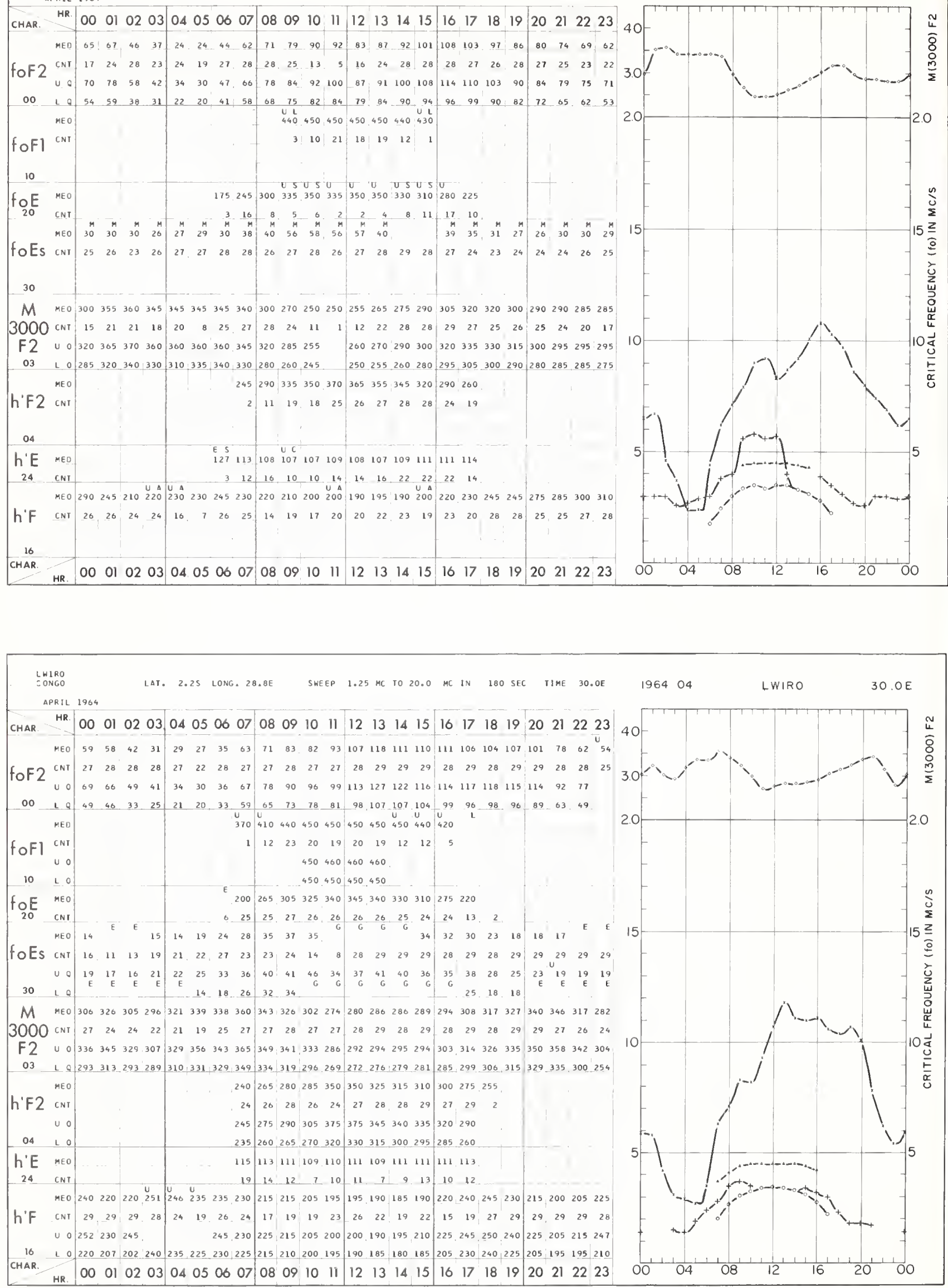
APRIL 1984

LAT. 12.0S LONG. 75.3M SWEEP 1.0 MC TO 25.0 MC IN 100 SEC TIME 75.0W 196404

HUANCAYO

75. OW

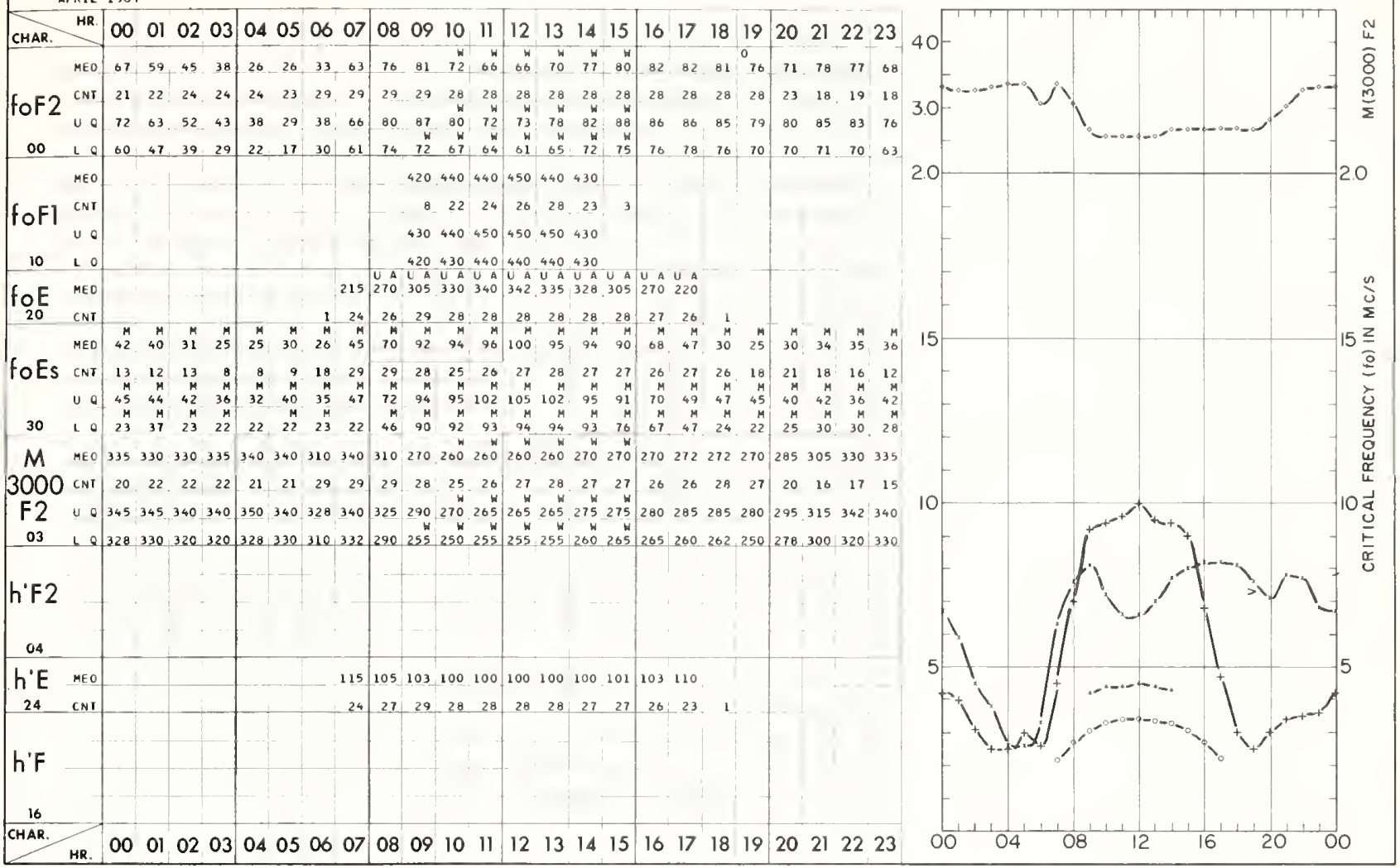

GOOLEY HEAO
NEW ZEALANO

LAT. 43.65 LONG.172.8E

SWEEP 1.0 MC IO 22.0 HC IN

196404

GODLEY HEAD

$180.0 E$

APRIL 1964

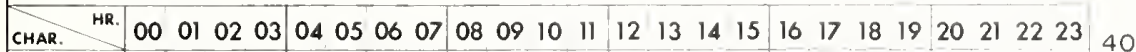

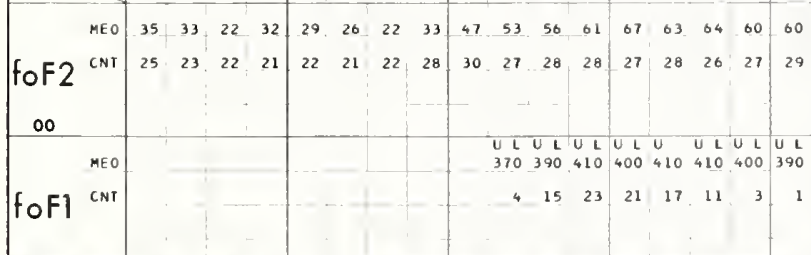

foE

S E E E E E E TI \begin{tabular}{llllll|llll|l|lll|llllllllllll}
20 & $\mathrm{CNT}$ & 4 & 7 & 7 & 6 & 10 & 9 & 10 & 26 & 27 & 27 & 27 & 27 & 28 & 28 & 26 & 25 & 27 & 24 & 17 & 1 & 1 & 3 & 4
\end{tabular} \begin{tabular}{|l|l|l|l|llll|l|llll|l|l|l|l|llll|llll} 
MEO & 22 & 16 & 21 & 22 & 20 & 24 & 24 & 24 & 25 & 32 & 36 & 31 & 33 & 30 & 31 & 28 & 24 & 22 & 16 & 24 & 26 & 23 & 25 & 2
\end{tabular}

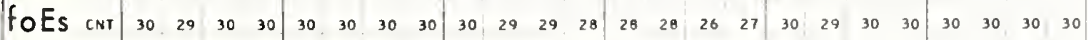
30

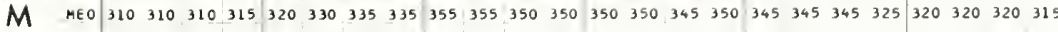

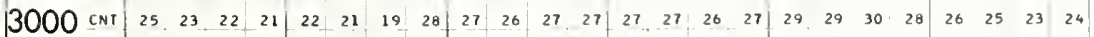

$\mathrm{F} 2$

0

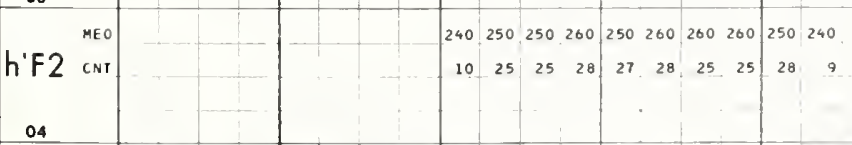

h'E MEO 24 CN

MED $270 \quad 260 \quad 260 \quad 260 \quad 260 \quad 250 \quad 250 \quad 240 \quad 230 \quad 220 \quad 200 \quad 200 \quad 200 \quad 200 \quad 210 \quad 220 \quad 230 \quad 240 \quad 220 \quad 240 \quad 240 \quad 250 \quad 260260$

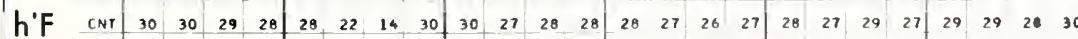

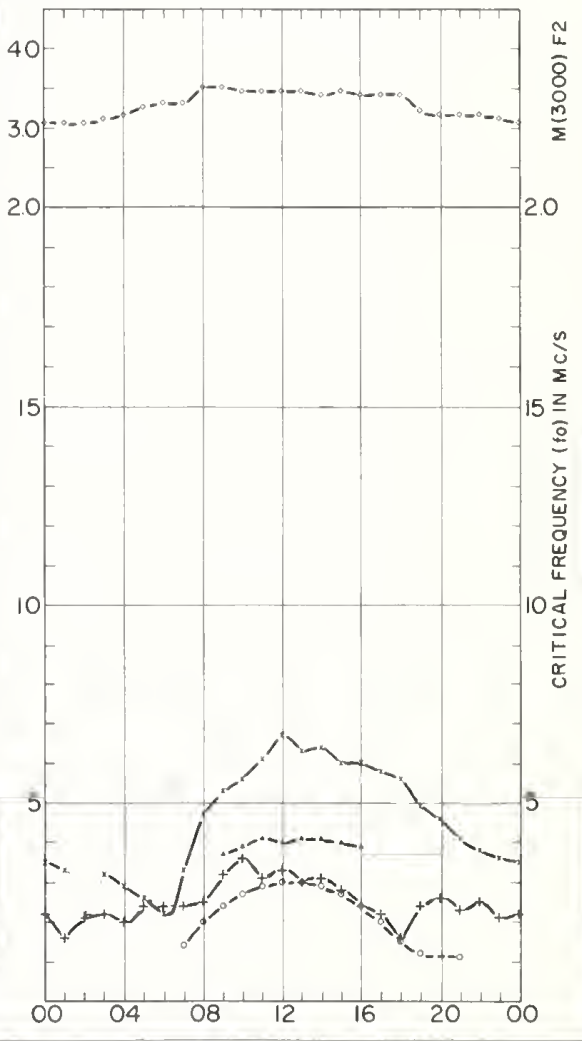



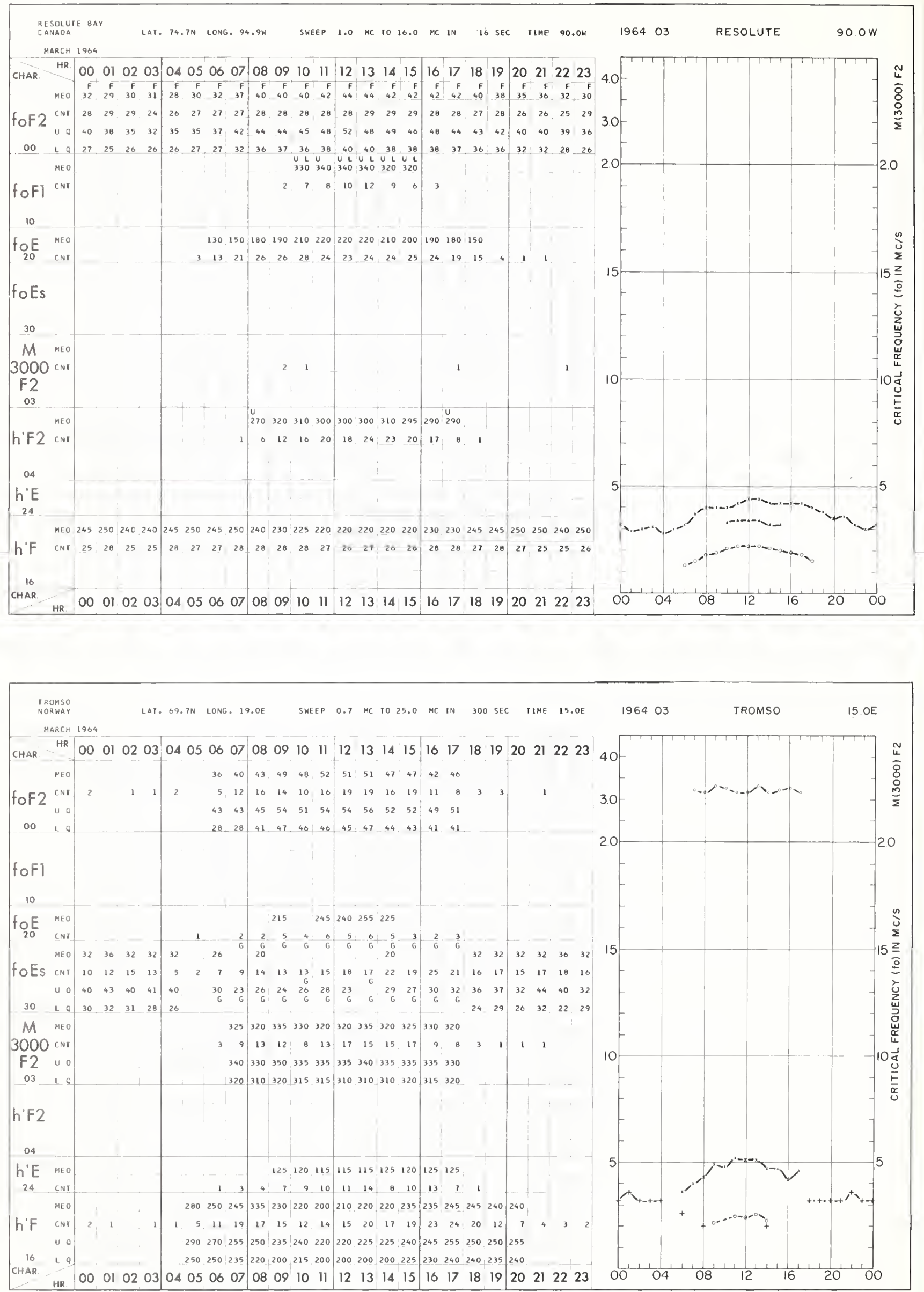


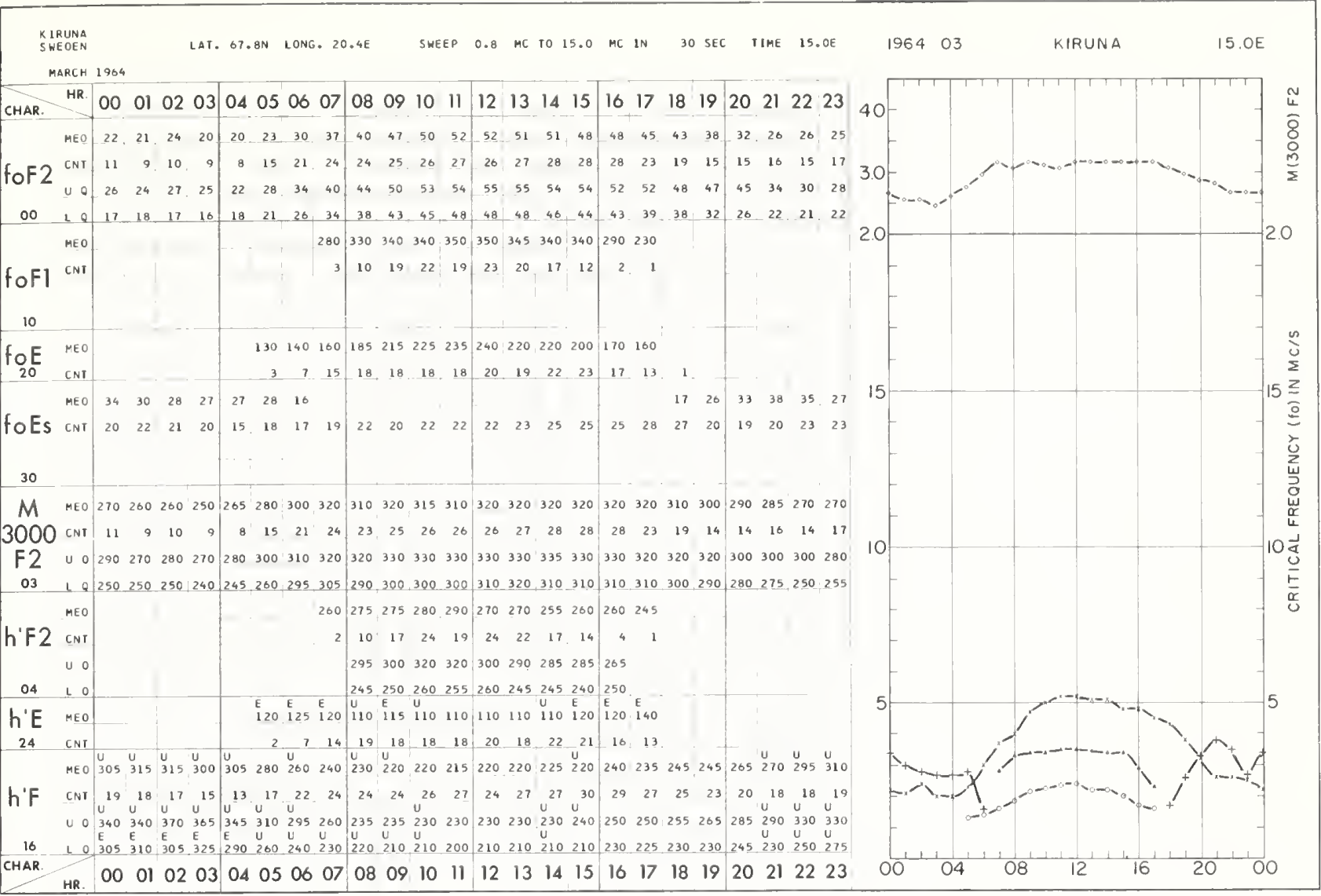

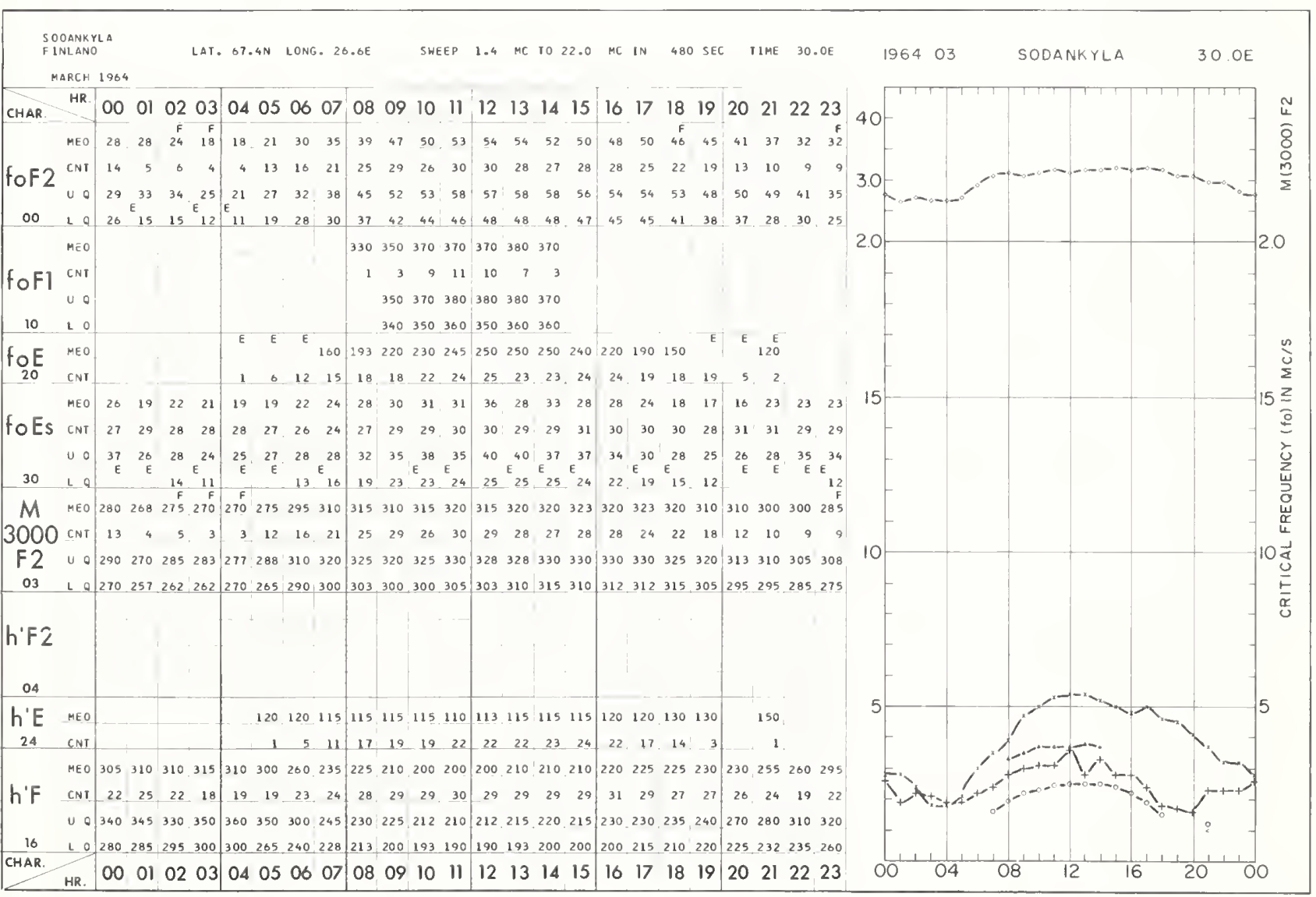




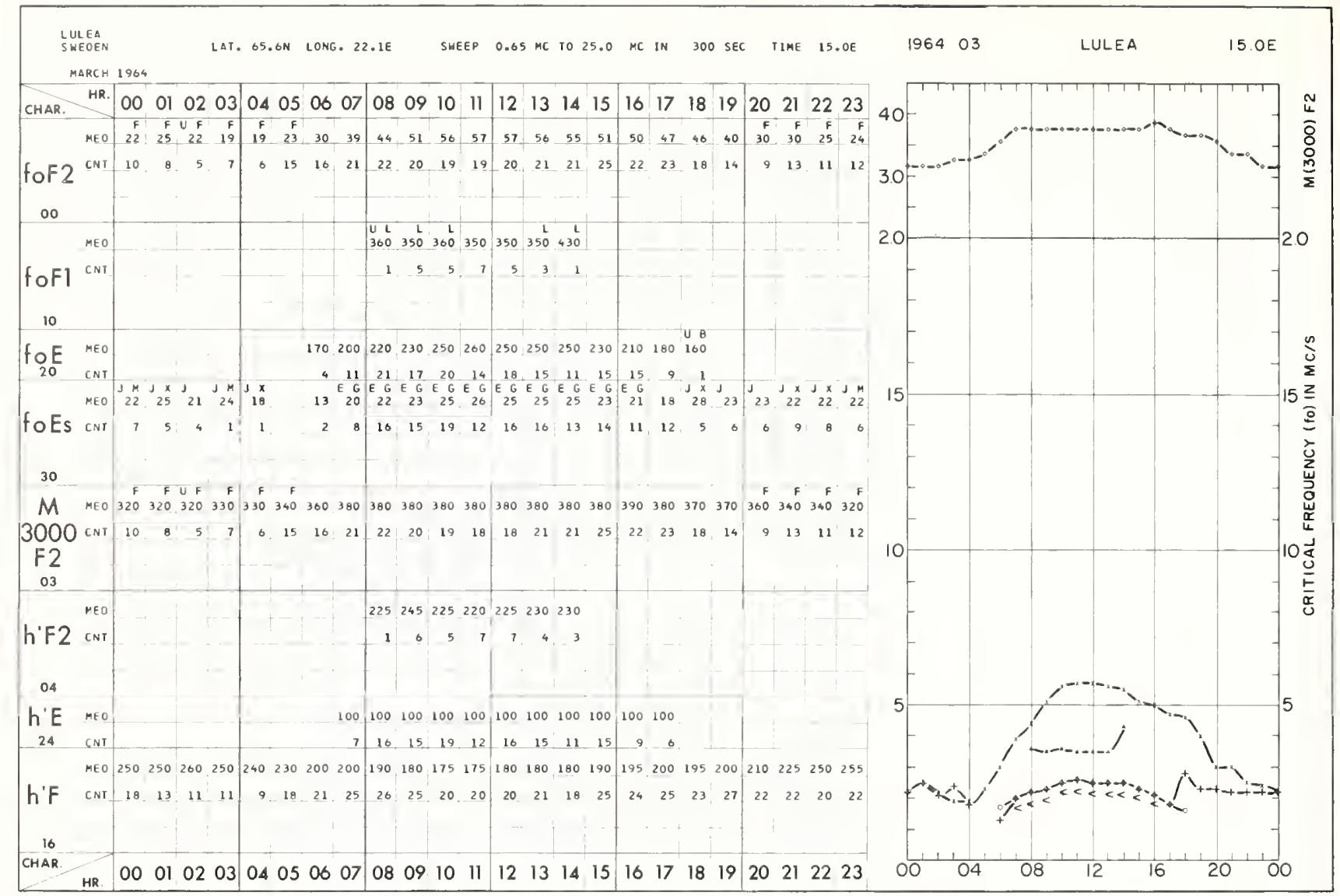

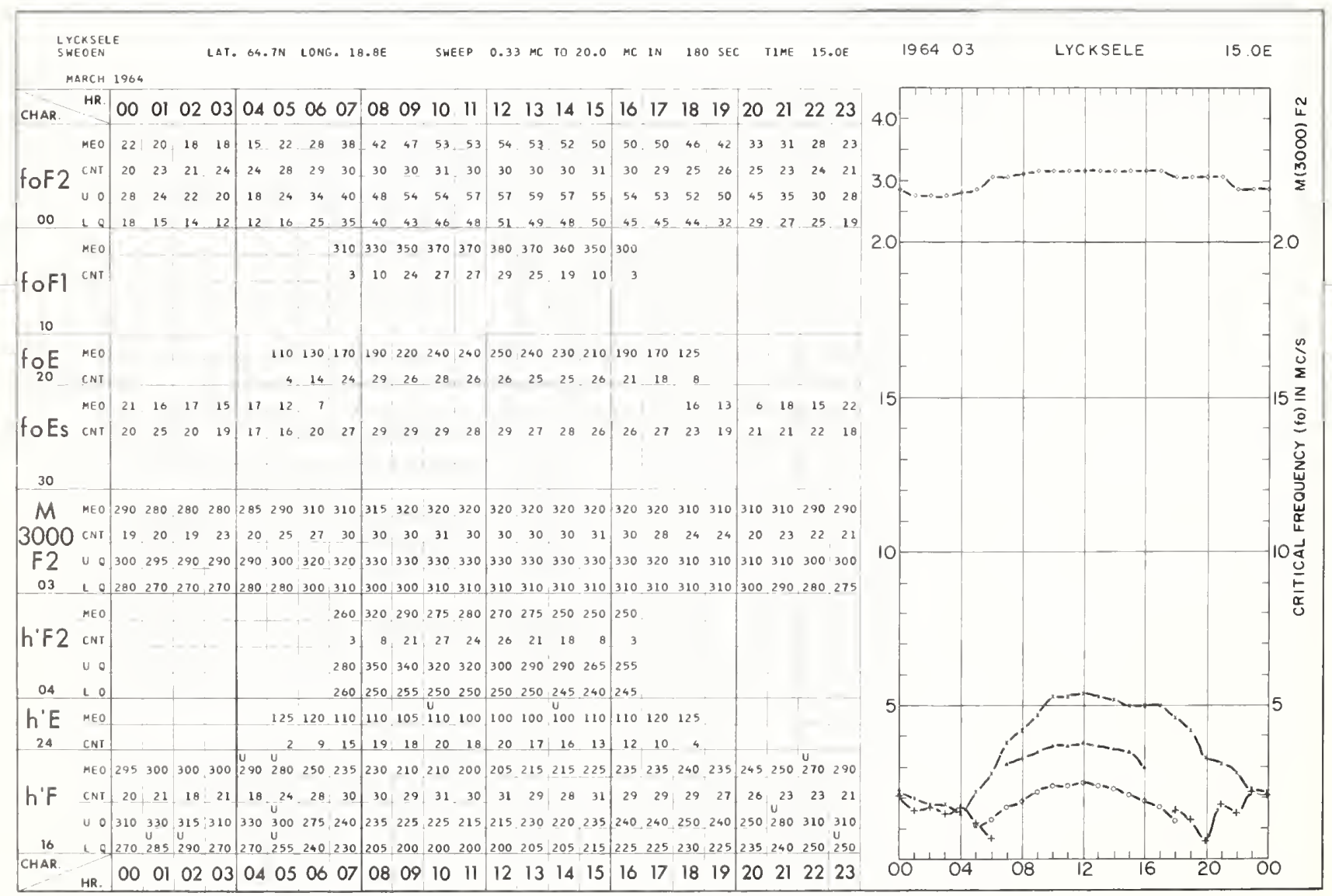



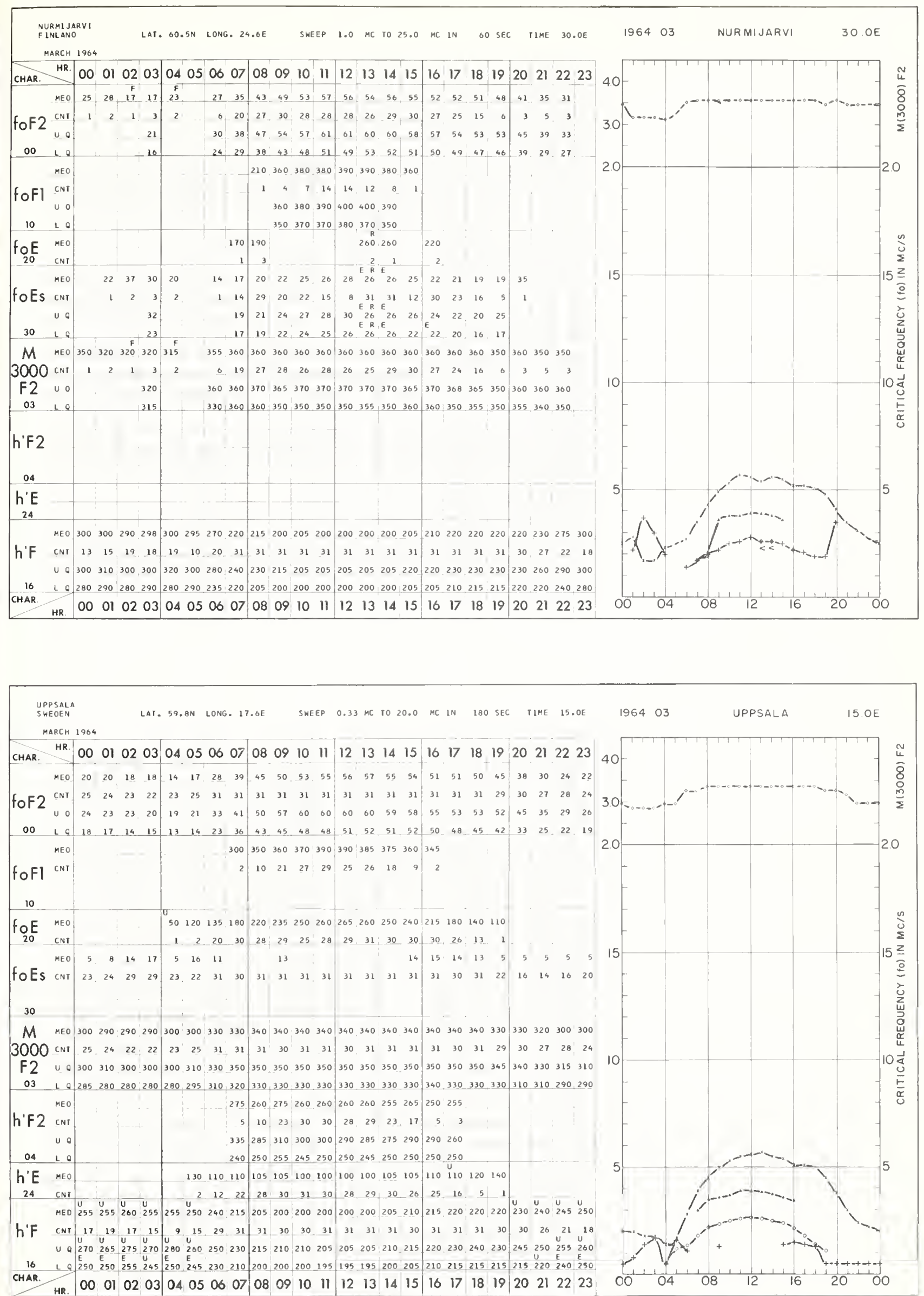

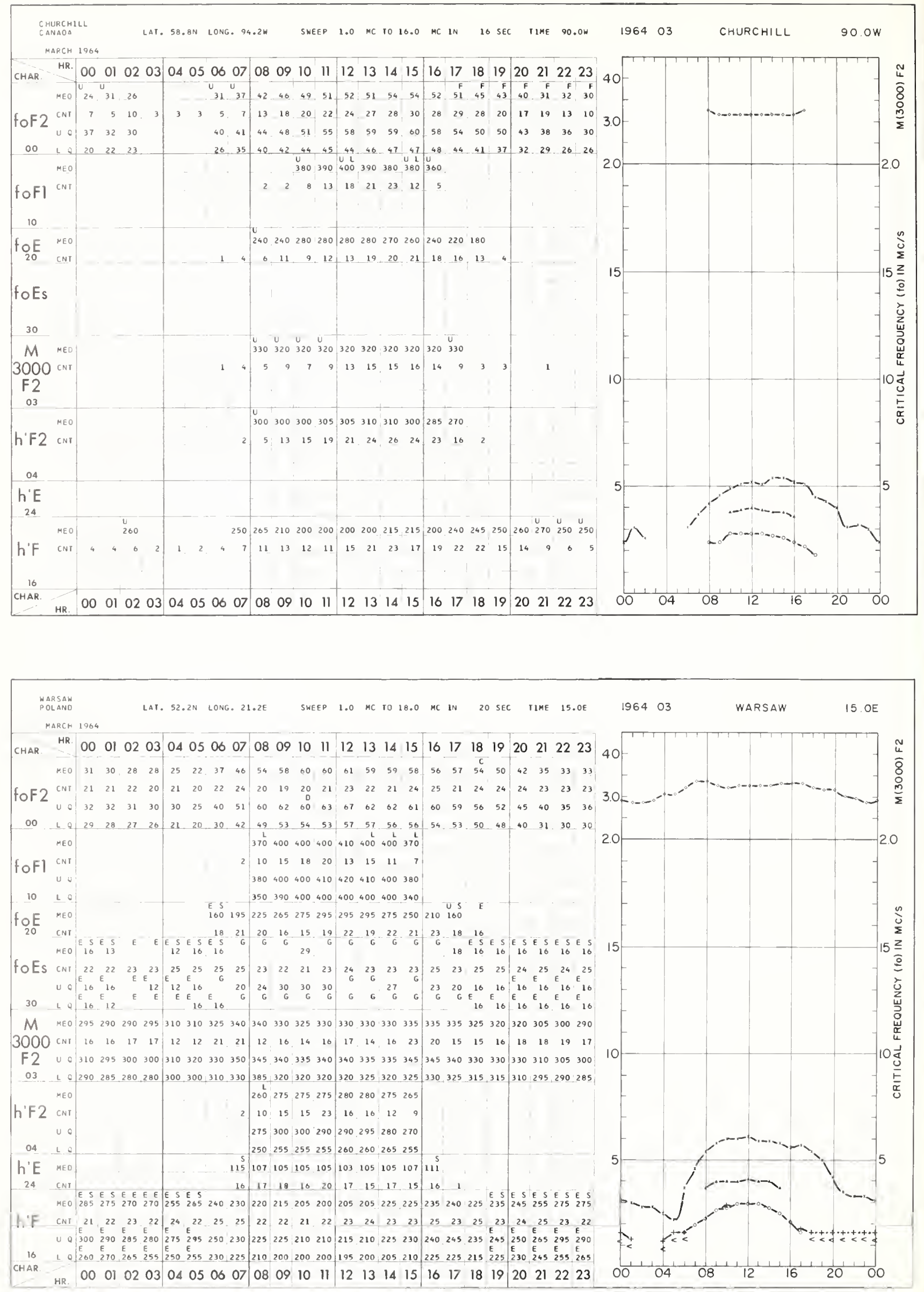
MARCH 1964

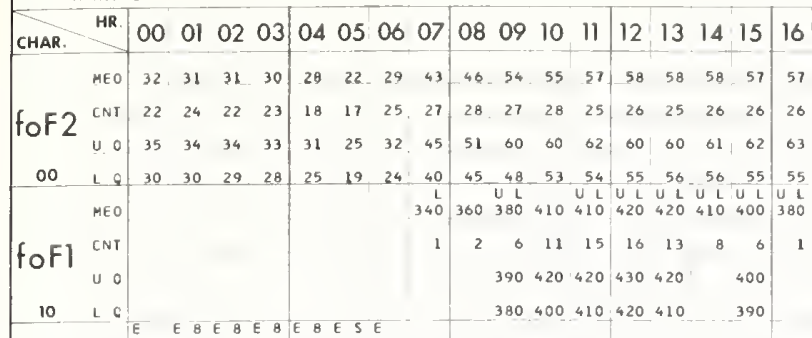

foE MEO ${ }^{E} 90$\begin{tabular}{c}
$E$ \\
\hline 0
\end{tabular} $\mathrm{O}_{20}$

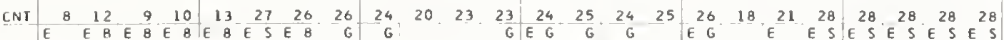

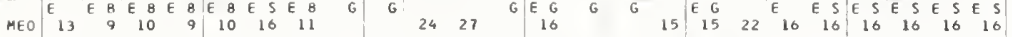

\begin{tabular}{|l|lllllllll|lllll|llllllllllll} 
foES CNT & 23 & 20 & 19 & 12 & 15 & 28 & 14 & 26 & 28 & 28 & 28 & 26 & 26 & 25 & 26 & 26 & 26 & 28 & 28 & 28 & 28 & 28 & 28 & 28
\end{tabular}

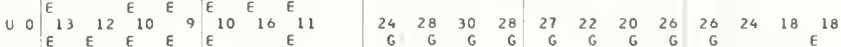

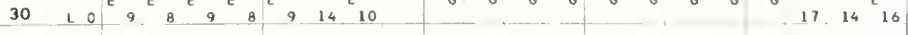

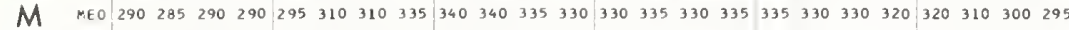
\begin{tabular}{|l|lllllllll|llllllllllllllll}
3000 & CNI & 22 & 24 & 22 & 22 & 17 & 13 & 24 & 27 & 27 & 26 & 28 & 24 & 26 & 25 & 26 & 26 & 26 & 28 & 28 & 28 & 26 & 25 & 23 & 22
\end{tabular}

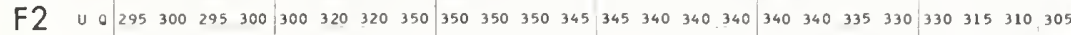

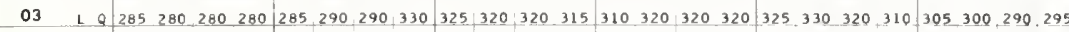

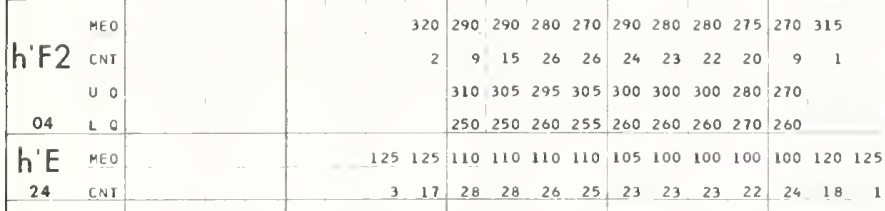

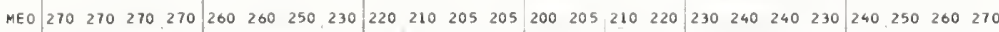

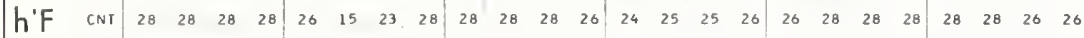
\begin{tabular}{ll|llll|l|l|l|l|l|l|l|l|l|l|l|l|l|l|l|l|l|l|l} 
U O 280 & 280 & 290 & 280 & 280 & 275 & 260 & 240 & 225 & 215 & 215 & 210 & 205 & 210 & 220 & 230 & 235 & 240 & 245 & 240 & 250 & 250 & 275 & 285
\end{tabular}

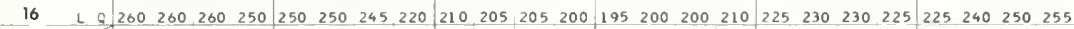
CHAR. HR. 00 \begin{tabular}{llll|llll|llll|llll|llll|llll}
01 & 02 & 03 & 04 & 05 & 06 & 07 & 08 & 09 & 10 & 11 & 12 & 13 & 14 & 15 & 16 & 17 & 18 & 19 & 20 & 21 & 22 & 23
\end{tabular}

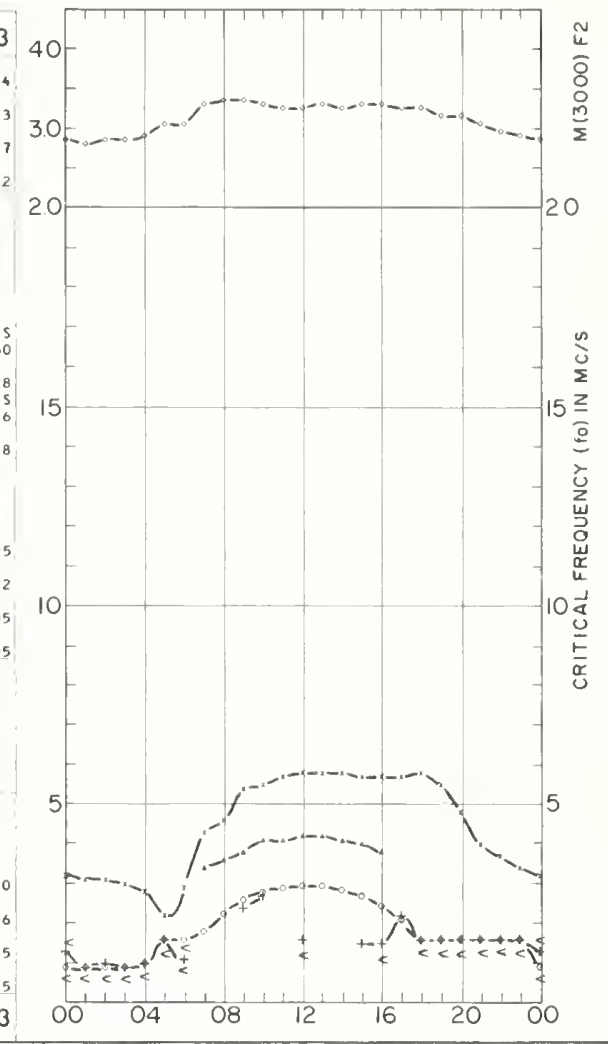

ODURBES
BELGIUM

LAT, 50.1N LONG. 4.6

SHEEP 1.0 MC TO 20.0 MC IN $180 \mathrm{SEC}$ TIME 0.0

196403

DOURBES

0.0

MARCH 1964

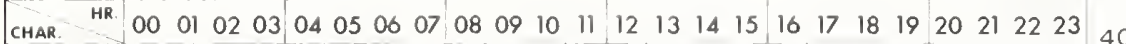

\begin{tabular}{l|l|lll|llll|lllllllll|llll|llll}
$M E 0$ & 31 & 30 & 30 & 28 & 26 & 23 & 35 & 44 & 51 & 56 & 58 & 60 & 58 & 58 & 60 & 59 & 57 & 55 & 57 & 52 & 44 & 36 & 33 & 32
\end{tabular}

foF 2

\begin{tabular}{llll|llll|llllllll|lllllllll}
33 & 32 & 32 & 31 & 28 & 25 & 39 & 51 & 58 & 60 & 61 & 62 & 62 & 60 & 63 & 61 & 60 & 64 & 63 & 57 & 50 & 40 & 37 & 35
\end{tabular}

\begin{tabular}{llllllllll|llll|llll|lllllllll}
00 & L & $Q$ & 30 & 29 & 28 & 26 & 23 & 20 & 30 & 42 & 46 & 50 & 56 & 56 & 56 & 56 & 57 & 56 & 55 & 53 & 53 & 48 & 38 & 32 & 31 & 30
\end{tabular} MEO $\quad 248335360380400405402400400360325$

foFl $\mathrm{CNI}$

\begin{tabular}{ll|llll|llll:l}
2 & 2 & 10 & 15 & 19 & 22 & 20 & 14 & 13 & 7 & 4
\end{tabular}

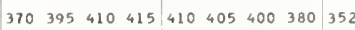

$10 \quad L 0$

\begin{tabular}{lllll|llll}
340 & 360 & 380 & 390 & 400 & 400 & 382 & 350 & 290
\end{tabular}

fOE

\begin{tabular}{ll|lllllllll|llll}
$E$ & $E$ & 105 & 180 & 220 & 248 & 265 & 280 & 290 & 280 & 265 & 240 & 210 & 170 & 105
\end{tabular}

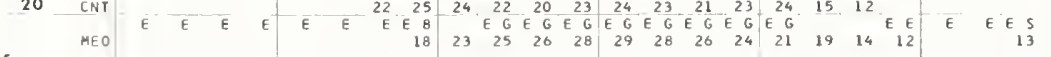

\begin{tabular}{llllll|llll|llll|lllllllllllll} 
foEs CNI & 28 & 28 & 28 & 28 & 28 & 28 & 26 & 26 & 27 & 25 & 26 & 26 & 26 & 23 & 24 & 24 & 26 & 26 & 26 & 26 & 27 & 27 & 28 & 28
\end{tabular}

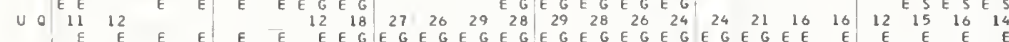
30 L

M MEO $300 \quad 297 \quad 297300 \quad 310 \quad 316 \quad 342 \quad 354 \quad 352 \quad 346 \quad 349 \quad 343 \quad 341 \quad 339338 \quad 344 \quad 338 \quad 340 \quad 339333 \quad 330 \quad 316 \quad 306 \quad 303$

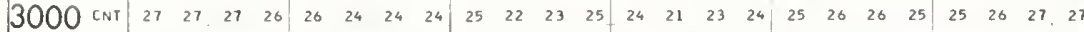

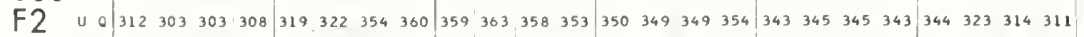

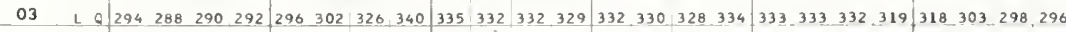
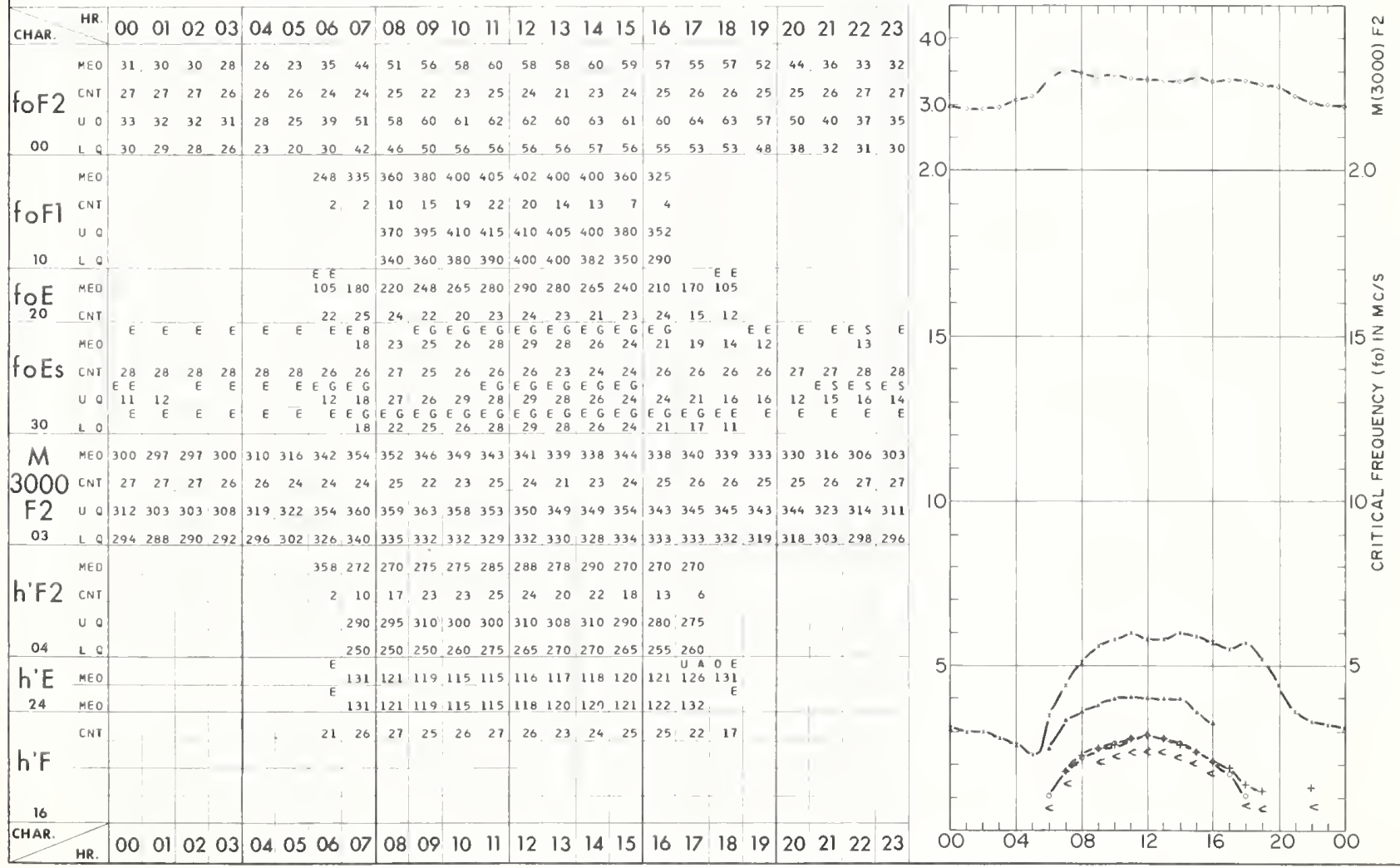


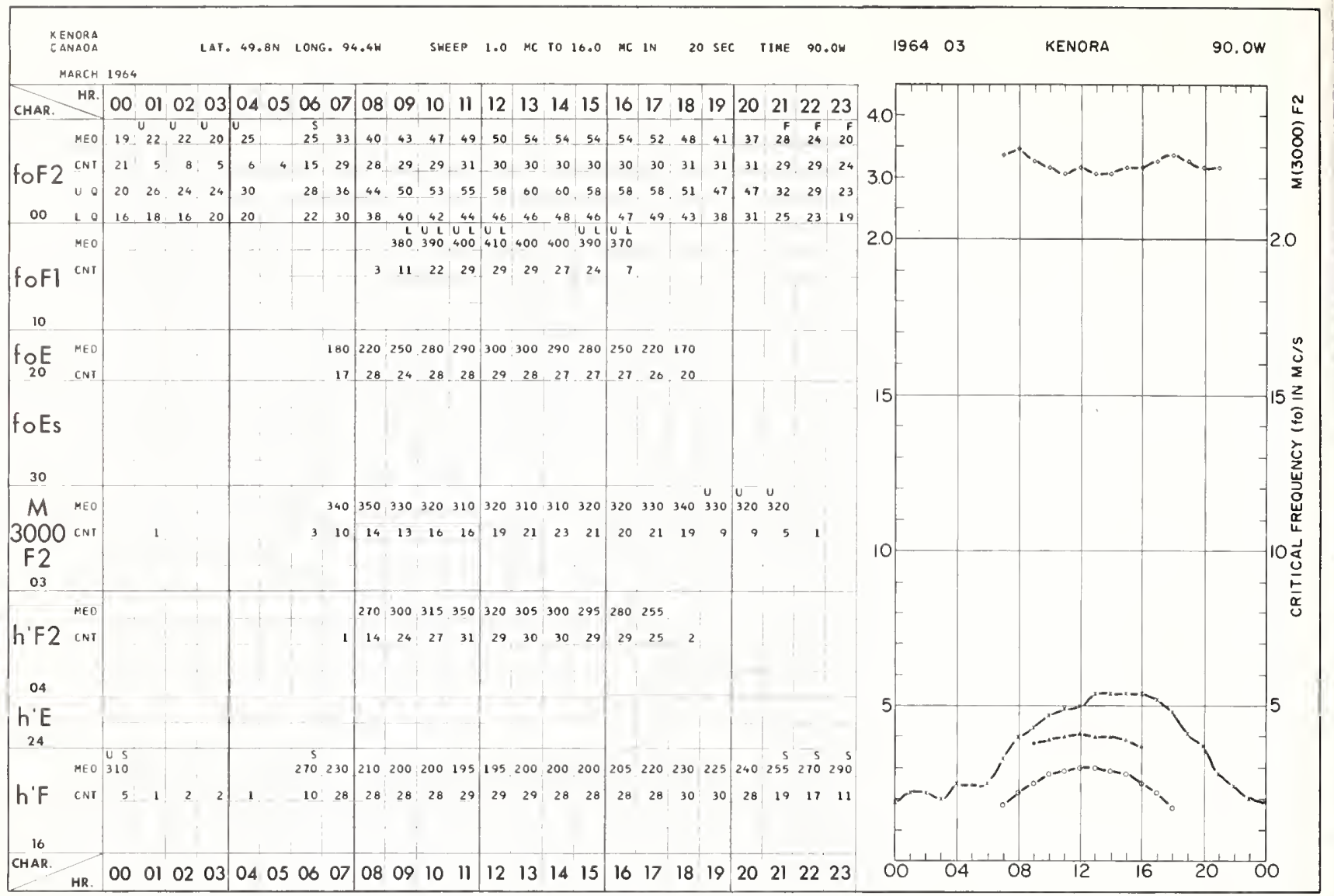

ST JOHNS

EEFOUNDLANO LAT. 47.6N LONG. 52.7H SWEEP 1.0 MC TO 16.0 MC IN 20 SEC TIME

\begin{tabular}{|l|l|lll|lllll|lllll|lllll|lllllllll}
\hline HR & 00 & 01 & 02 & 03 & 04 & 05 & 06 & 07 & 08 & 09 & 10 & 11 & 12 & 13 & 14 & 15 & 16 & 17 & 18 & 19 & 20 & 21 & 22 & 23
\end{tabular}

CHAR

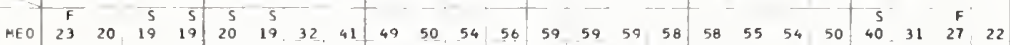

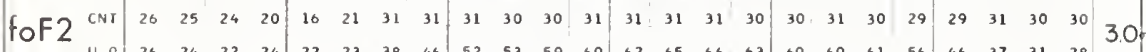

\begin{tabular}{llllll|lll|l|lll|l|l|llll|lllllllll}
00 & $L$ & 18 & 18 & 18 & 16 & 16 & 17 & 27 & 40 & 43 & 47 & 48 & 50 & 52 & 53 & 52 & 52 & 53 & 52 & 50 & 42 & 32 & 27 & 22 & 20
\end{tabular}

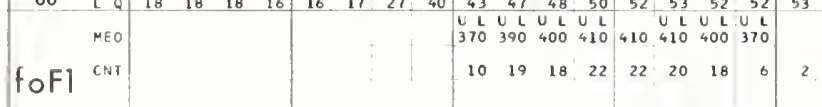

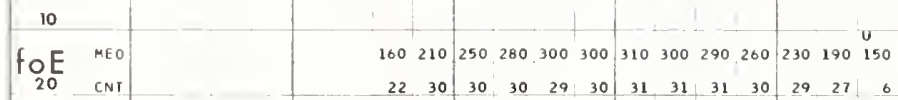

\section{foEs}

ME

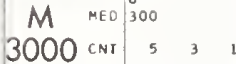

$\mathrm{F} 2$

MEO

h'F2 ${ }_{\mathrm{CNI}}^{\text {MEO }}$

\begin{tabular}{l|l|l|l|l|l|l|l|l|l|ll}
\hline & $U$ & $U$ & \\
\hline & 270 & 285 & 300 & 300 & 305 & 300 & 300 & 300 & 280 & 280 & 280
\end{tabular}

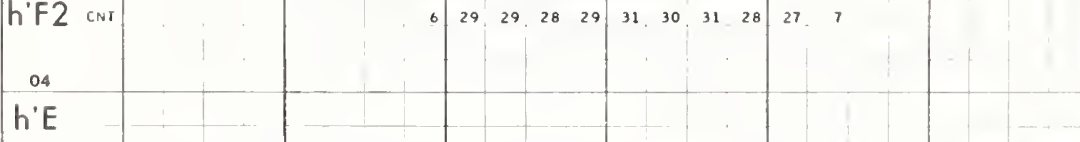

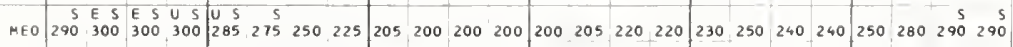

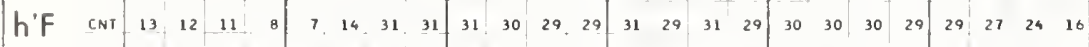

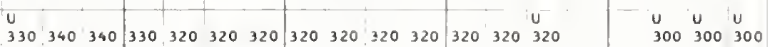
\begin{tabular}{llll|llll|llll|llll|llll}
1 & 6 & 19 & 19 & 17 & 18 & 21 & 24 & 24 & 28 & 27 & 25 & 21 & 18 & 7 & 4 & 1 & 8 & 6 & 6
\end{tabular}

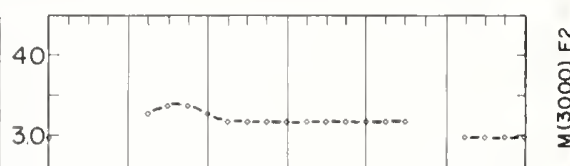


WAKKANAL
JAPAN

LAT. 45.4N LONG.141.7E SWEEP 1.0 MC TO 18.0 MC IN 40 SEC TIME 135.0 E

196403

WAKKANAI

$135.0 E$

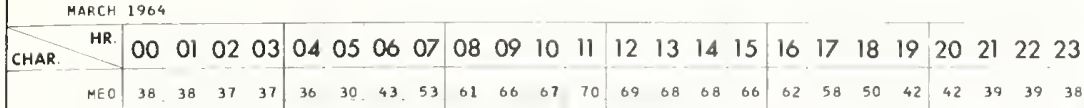
foF2 \begin{tabular}{l|llll|llll|llll|llll|llll|llll} 
CNI & 19 & 19 & 19 & 19 & 18 & 25 & 27 & 28 & 27 & 28 & 30 & 30 & 31 & 30 & 30 & 30 & 30 & 30 & 30 & 30 & 29 & 28 & 25 & 22
\end{tabular}

\begin{tabular}{ll|llll|llll|lllllllllllllll|llll}
40 & 41 & 40 & 41 & 40 & 36 & 46 & 56 & 65 & 71 & 73 & 73 & 73 & 75 & 71 & 68 & 63 & 60 & 57 & 53 & 51 & 40 & 44 & 42 \\
00 & $\mathrm{~L}$ & 0 & 35 & 36 & 34 & 34 & 34 & 28 & 38 & 50 & 54 & 60 & 62 & 63 & 64 & 65 & 65 & 63 & 58 & 54 & 46 & 36 & 37 & 36 & 36 & 36
\end{tabular}

\begin{tabular}{rr|r|rrrrrrrr|r} 
& MEO & 350 & 400 & 400 & 420 & 430 & 430 & 420 & 410 & 390 & 330 \\
\hline foFl CNI & 1 & 12 & 24 & 29 & 28 & 31 & 29 & 29 & 27 & 3
\end{tabular}

FOE

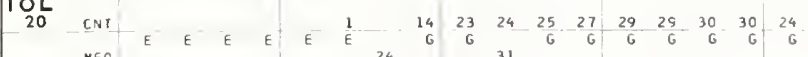

\begin{tabular}{|l|l|l|l|l|lllllllllllll|llllllll}
\hline foES CNI & 30 & 30 & 29 & 29 & 29 & 24 & 3 & 18 & 27 & 26 & 28 & 29 & 31 & 30 & 30 & 29 & 27 & 13 & 14 & 28 & 30 & 29 & 30 & 30
\end{tabular} $\begin{array}{lllllllllllllllllllllllllllllll}U & E & E & E & 21 & 20 & E & 30 & 26 & 32 & 35 & 34 & G & 38 & 33 & G & 26 & 30 & 34 & 31 & 30 & E & E & E\end{array}$

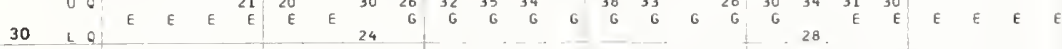

$M \quad$ MEO $300 \quad 300 \quad 305 \quad 310 \quad 325 \quad 325 \quad 345 \quad 350 \quad 340 \quad 340 \quad 335 \quad 330 \quad 330 \quad 335 \quad 335 \quad 340 \quad 345 \quad 345 \quad 340 \quad 315 \quad 310 \quad 310 \quad 305 \quad 300$

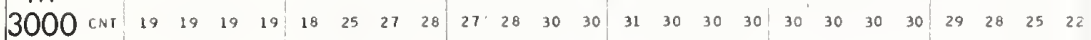

\section{F2}

$\begin{array}{ll}03 & \\ \text { MEO }\end{array}$

h'F2 CNI

\begin{tabular}{l|llll|l|l|l|l|l|l}
350 & 270 & 260 & 265 & 270 & 275 & 275 & 270 & 255 & 250
\end{tabular}

\begin{tabular}{llll|llll|l}
12 & 24 & 28 & 27 & 31 & 29 & 29 & 27 & 5
\end{tabular}

h'E

24

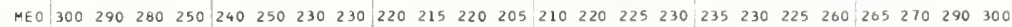

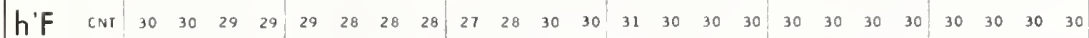

$\begin{array}{llllllllllllllllllllllllll}00 & 01 & 02 & 03 & 04 & 05 & 06 & 07 & 08 & 09 & 10 & 11 & 12 & 13 & 14 & 15 & 16 & 17 & 18 & 19 & 20 & 21 & 22 & 23\end{array}$

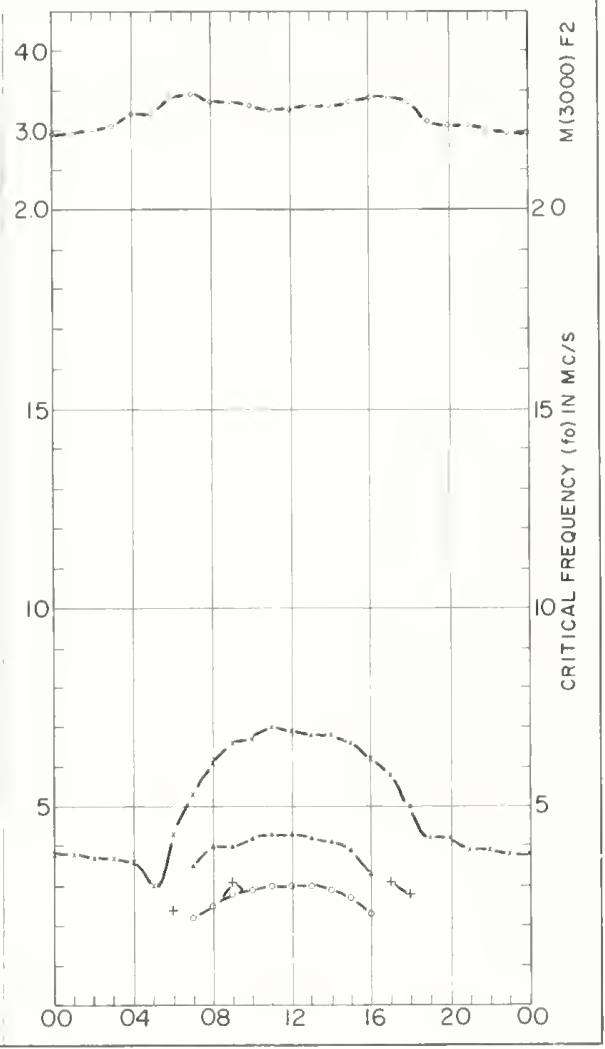

OIIAWA
CANAOA

MARCH 1964

LAT. 45.4N LONG. 75.9W SWEEP 1.0 MC TO 16.0 MC IN 16 SEC TLME $75.0 \mathrm{~W} 1964$ O3

$75.0 \mathrm{~W}$

$\begin{array}{llllllllllllllllllllllllllll}\text { CHAR. } & \text { HR. } & 00 & 01 & 02 & 03 & 04 & 05 & 06 & 07 & 08 & 09 & 10 & 11 & 12 & 13 & 14 & 15 & 16 & 17 & 18 & 19 & 20 & 21 & 22 & 23\end{array}$

foF2 CNT 26 \begin{tabular}{llllllllllll|lllllllllllll} 
& 24 & 19 & 11 & 8 & 12 & 24 & 30 & 30 & 30 & 31 & 31 & 31 & 31 & 31 & 31 & 31 & 31 & 31 & 30 & 30 & 29 & 29 & 29 & 30
\end{tabular}

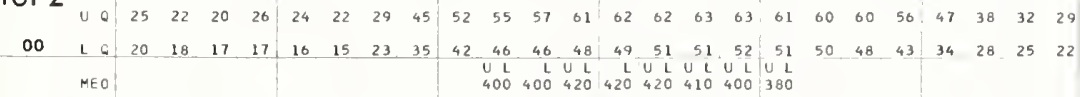

\section{foFl CNT}

\begin{tabular}{|ll|}
10 & \\
\hline FOE & MEO \\
\hline 20 & $C N T$
\end{tabular}

\begin{tabular}{llllllll|l}
4 & 14 & 23 & 28 & 27 & 26 & 25 & 17 & 6
\end{tabular}

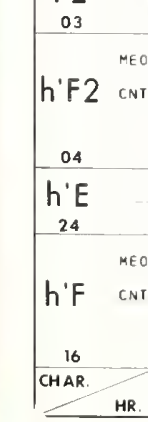

\begin{tabular}{l}
\hline h' \\
\hline h' \\
\hline $\mathrm{CH}$ \\
\hline
\end{tabular}

\section{foEs \\ M MEO \\ $300 \mathrm{CNT}^{2}$ \\ F2}

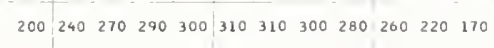
\begin{tabular}{ll|llllllllllll}
3 & 26 & 29 & 29 & 31 & 30 & 29 & 30 & 29 & 29 & 27 & 27 & 15
\end{tabular}

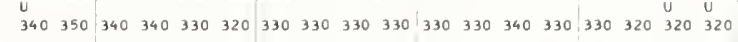
\begin{tabular}{ll|lllllllllllllll}
5 & 19 & 22 & 21 & 23 & 25 & 26 & 27 & 28 & 30 & 31 & 30 & 26 & 19 & 16 & 14 & 9
\end{tabular} $255,280300,300 \quad 300300295 \quad 290 \quad 270260$

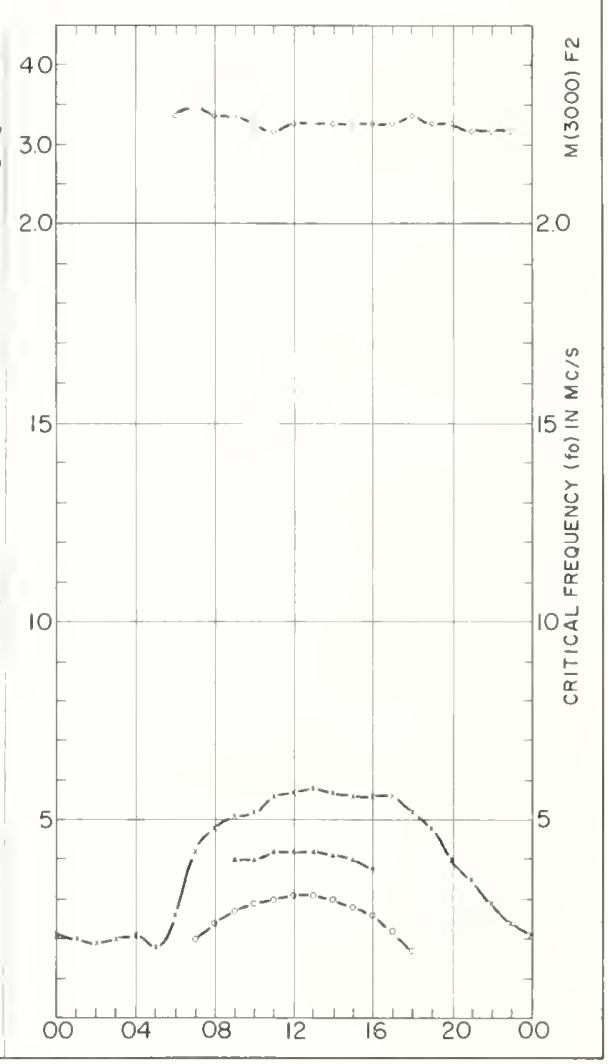




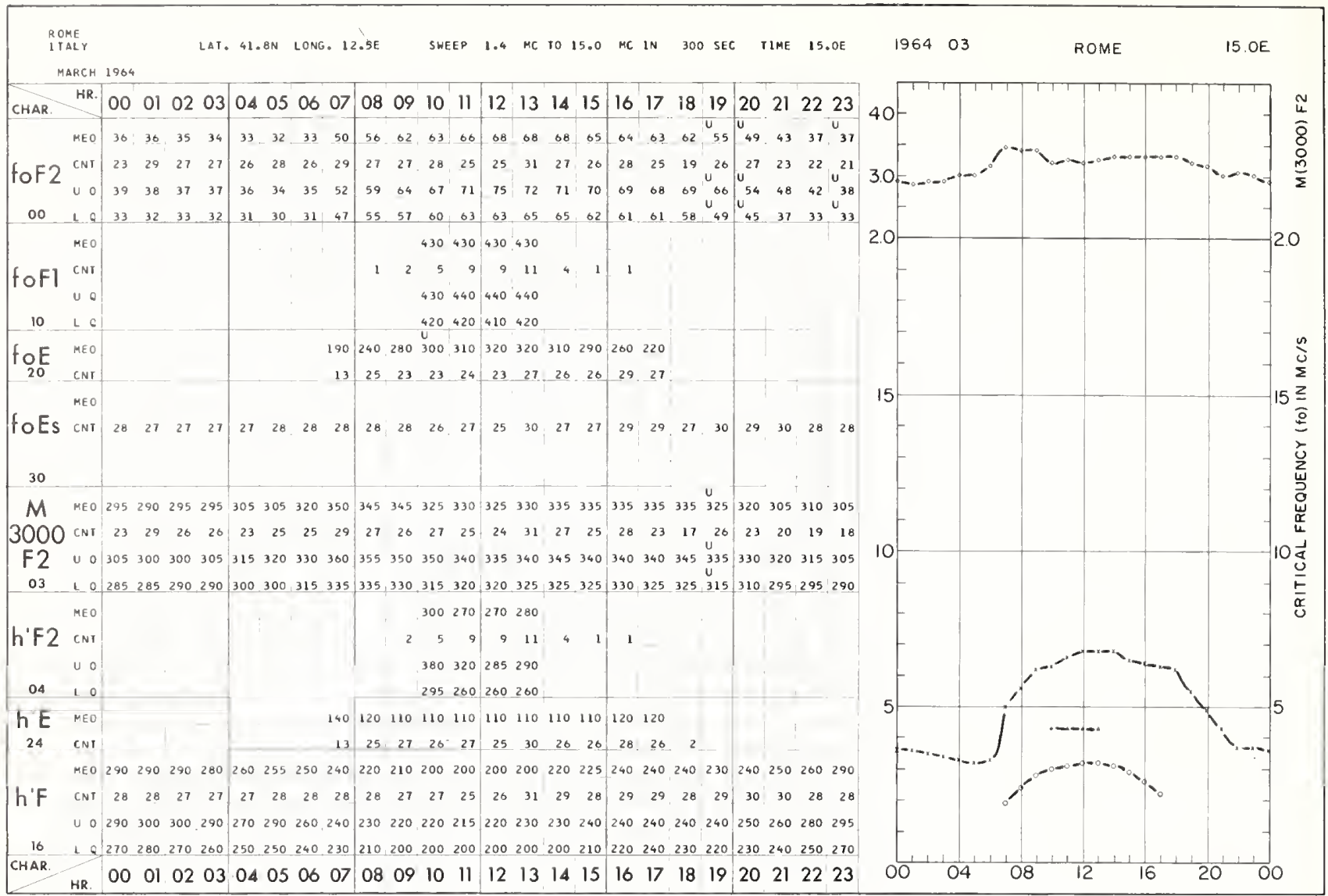

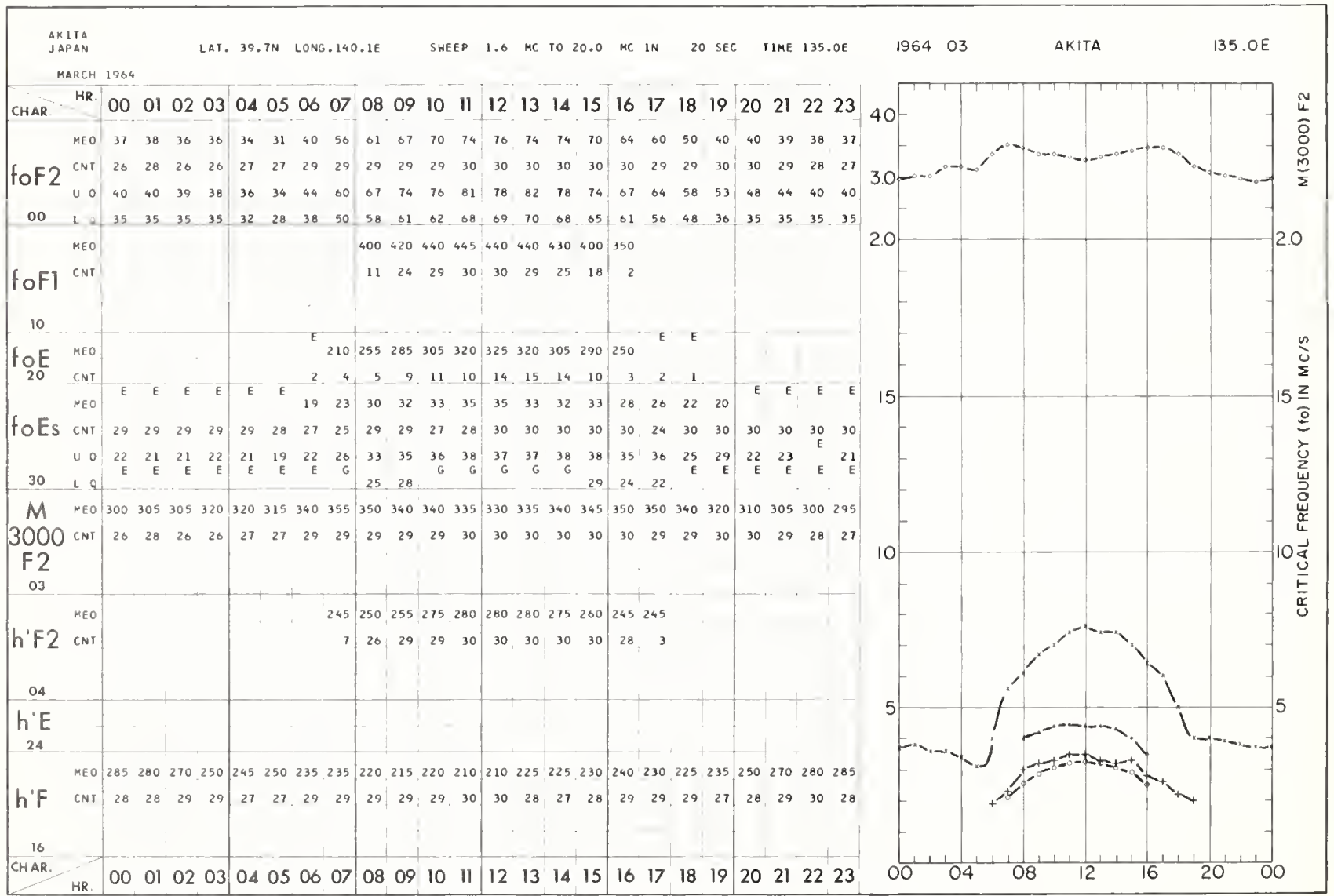


MARCH 1964

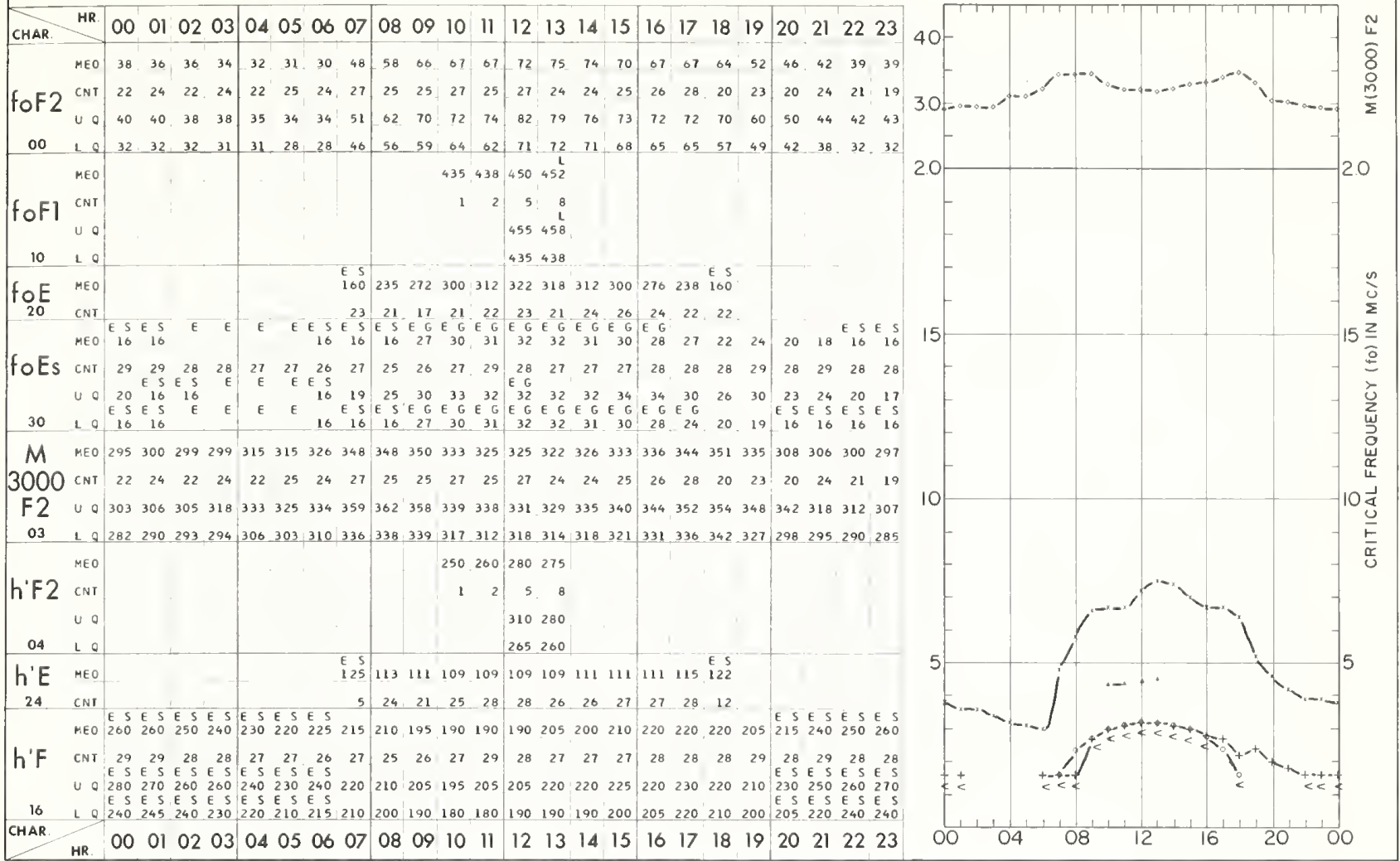

KOKUAUNJI
JAPAN

MARCH 1964

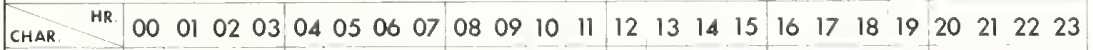
\begin{tabular}{r|llll|llll|llll|lllll|llllllll} 
MEO & 35 & 35 & 34 & 36 & 30 & 28 & 39 & 50 & 02 & 09 & 72 & 80 & 79 & 81 & 76 & 73 & 67 & 62 & 53 & 42 & 36 & 36 & 36 & 36 \\
fOF2 CNI & 30 & 29 & 28 & 30 & 29 & 30 & 29 & 30 & 29 & 26 & 28 & 28 & 29 & 30 & 30 & 31 & 31 & 31 & 30 & 28 & 29 & 29 & 31 & 30
\end{tabular} foF 2 \begin{tabular}{lllll:llll|lllllllll|llll|lllll}
4 & 8 & 38 & 38 & 38 & 38 & 32 & 31 & 44 & 59 & 66 & 73 & 78 & 85 & 88 & 88 & 86 & 79 & 74 & 68 & 63 & 50 & 44 & 40 & 38 & 38
\end{tabular}

\begin{tabular}{llllll|lllllllll|llll|llllllll}
00 & 2 & 9 & 33 & 33 & 33 & 33 & 28 & 26 & 36 & 52 & 57 & 62 & 66 & 71 & 71 & 74 & 70 & 67 & 63 & 59 & 45 & 36 & 32 & 32 & 33 & 33
\end{tabular} FOFI CNT

10

\begin{tabular}{cc} 
fOE \\
20 & MEO \\
\hline CN &
\end{tabular}

\begin{tabular}{llllllllll|ll}
$130 \quad 160220$ & 255 & 285 & 310 & 320 & 325 & 320 & 310 & 290 & 250 & 205
\end{tabular}

\begin{tabular}{llllllllll|llllllll|lllllllllllll}
$M E O$ & 20 & 22 & 20 & 18 & 13 & $E$ & 19 & $G$ & $G$ & 30 & 33 & 32 & 36 & 35 & 34 & 32 & 31 & 28 & 29 & 25 & 28 & 24 & 20 & 21
\end{tabular} \begin{tabular}{llllll|llll|lllll|lllllllll|lll} 
foES CNI & 27 & 26 & 28 & 29 & 28 & 29 & 22 & 30 & 28 & 26 & 26 & 26 & 29 & 29 & 30 & 31 & 31 & 31 & 27 & 27 & 28 & 25 & 29 & 24
\end{tabular} \begin{tabular}{rrrrrr|rrrr|rrrrr|rrrrr|rrrrrrrrrrr} 
& $U$ & $G$ & 22 & 24 & 23 & 23 & 19 & 20 & 22 & 25 & 30 & 35 & 37 & 39 & 38 & 38 & 37 & 39 & 37 & 38 & 36 & 39 & 41 & 36 & 26 & 28 \\
30 & $L$ & 0 & $E$ & $E$ & $E$ & $E$ & $E$ & $E$ & $G$ & $G$ & $G$ & $G$ & $G$ & $G$ & $G$ & 33 & $G$ & $G$ & 25 & 19 & 21 & 21 & 18 & 20 & $E$ & 20
\end{tabular}

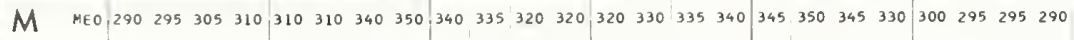
\begin{tabular}{|l|llll|llll|l|lll|ll|ll|l|lll|llll}
$3000 \mathrm{CNJ}$ & 30 & 29 & 28 & 30 & 29 & 30 & 29 & 30 & 29 & 26 & 28 & 28 & 29 & 30 & 30 & 31 & 31 & 31 & 30 & 27 & 29 & 28 & 31 & 30
\end{tabular} F2

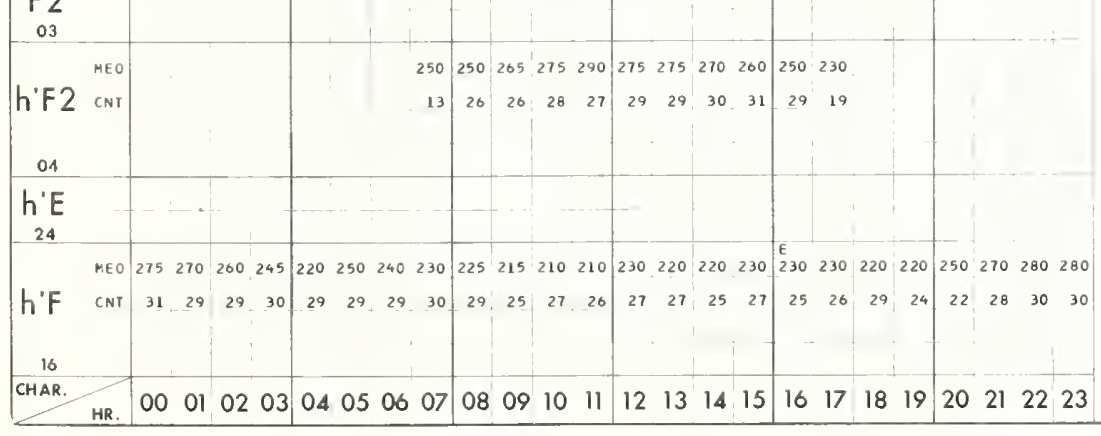

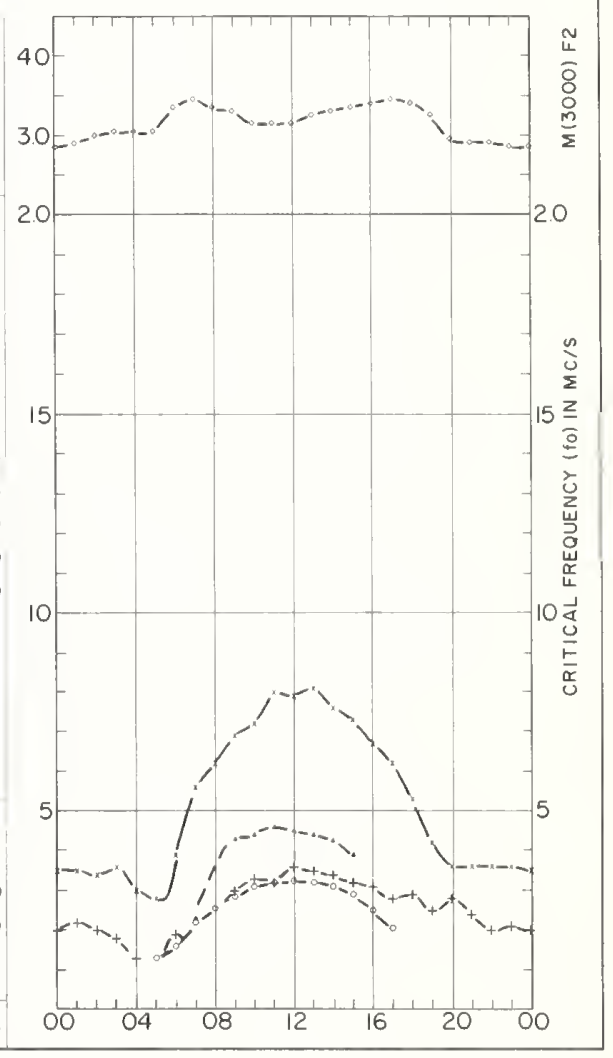




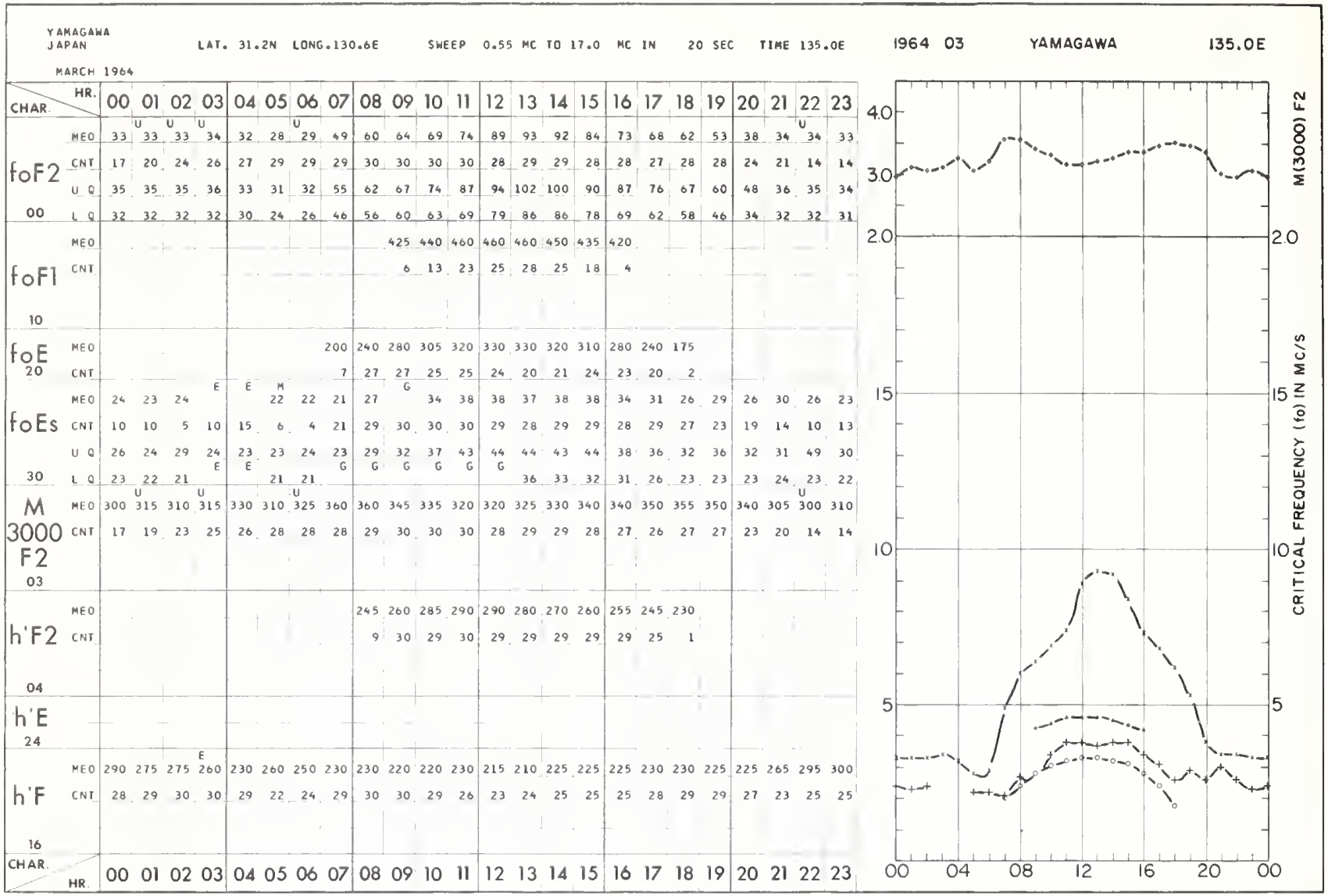

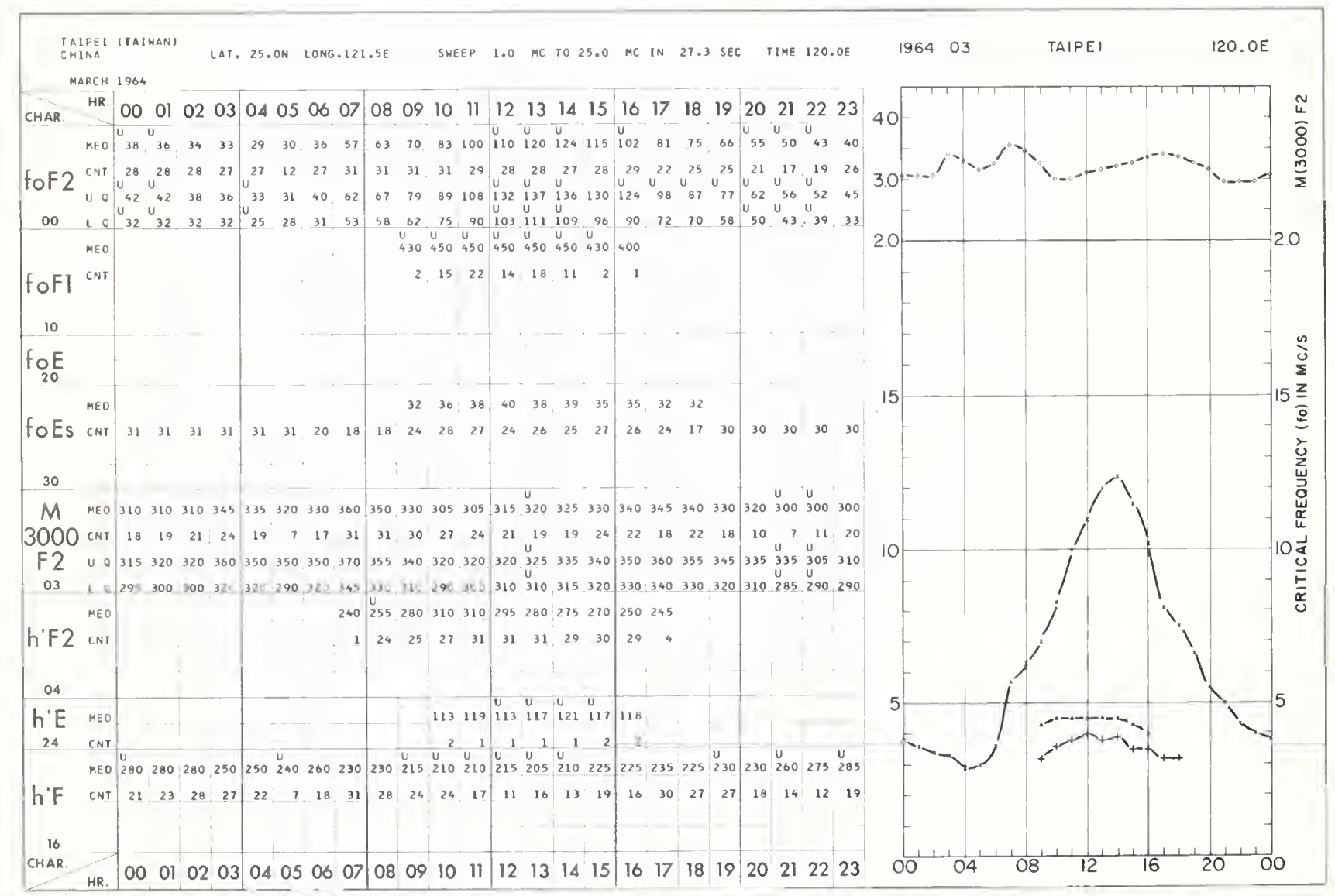


MANILA

LAT. 14.7N LONG.121.16

SHEEP 0.25 HC TO 20.0 HC IN 27 SEC TIME 120.0 E

196403

MANILA

$120.0 E$

MARCH 1964

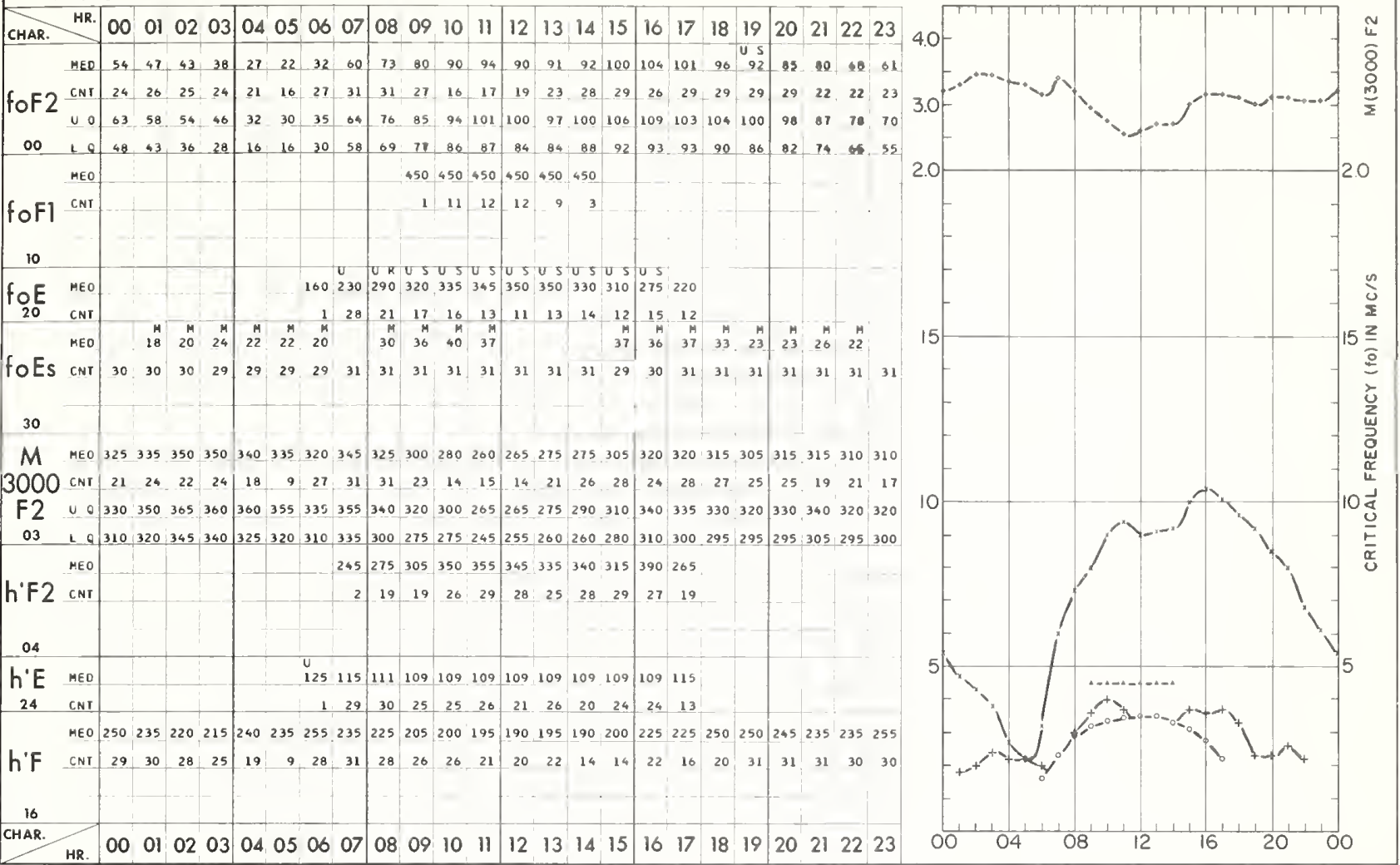

SINGAPORE
MALAYSIA

MARCH 1964

LAT. 1.3N LONG.103.8E

SHEEP 0.67 HC 1025.0 HC IN 300 SEC TIME 105.0 E

1964 O3 SINGAPORE

105.OE

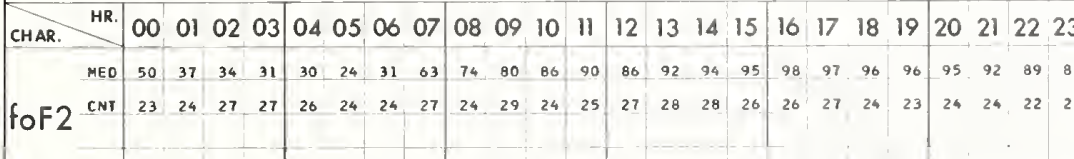

00

foFl

10

fog

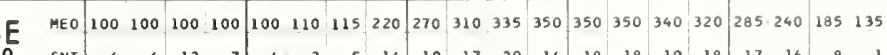

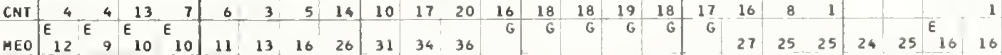

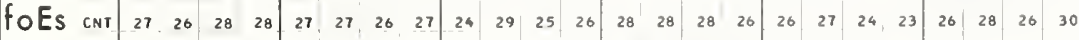

30

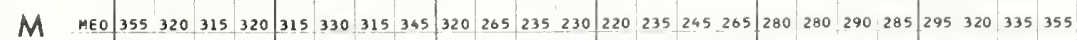

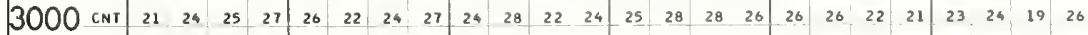
F2

o9

h'F2
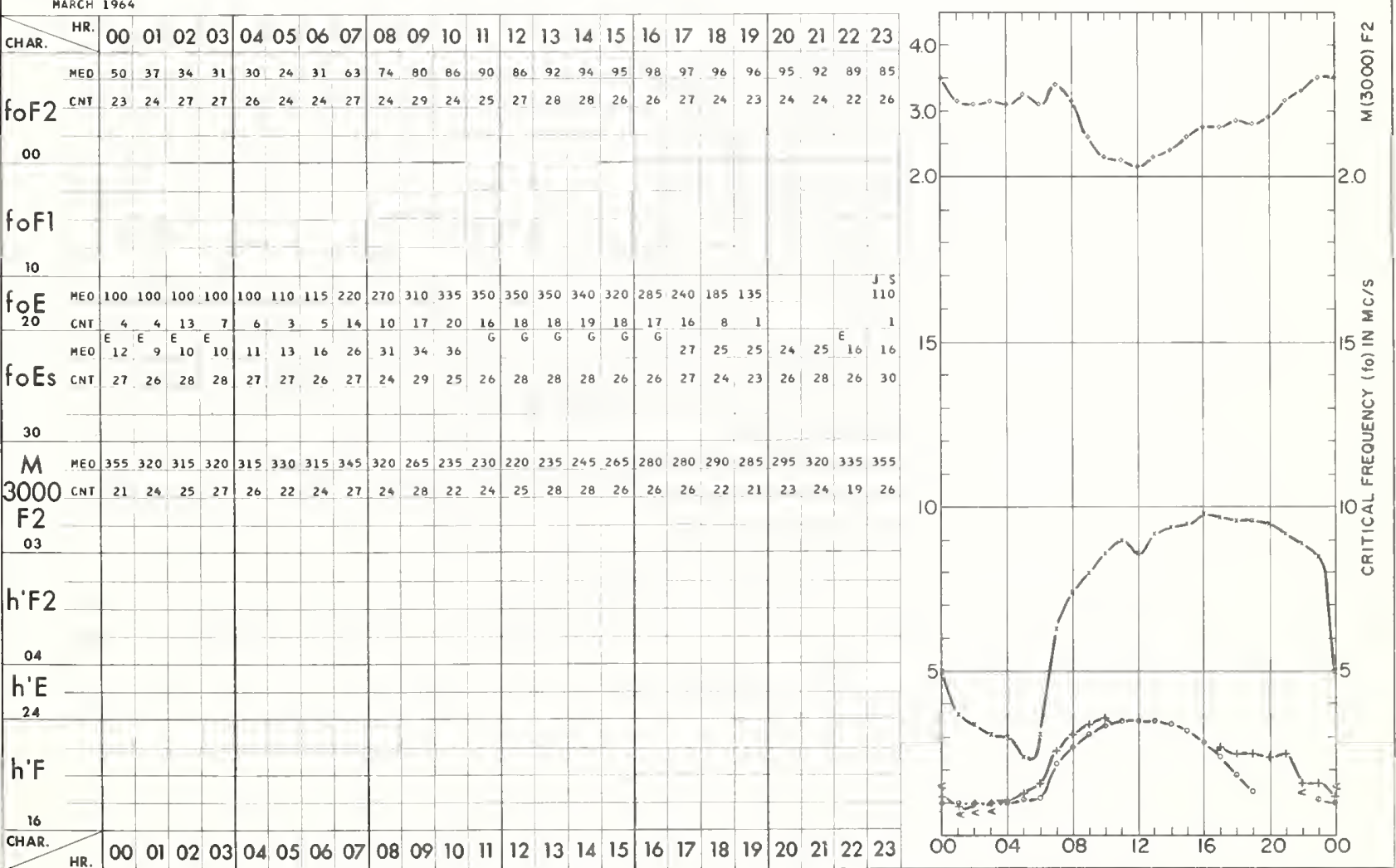


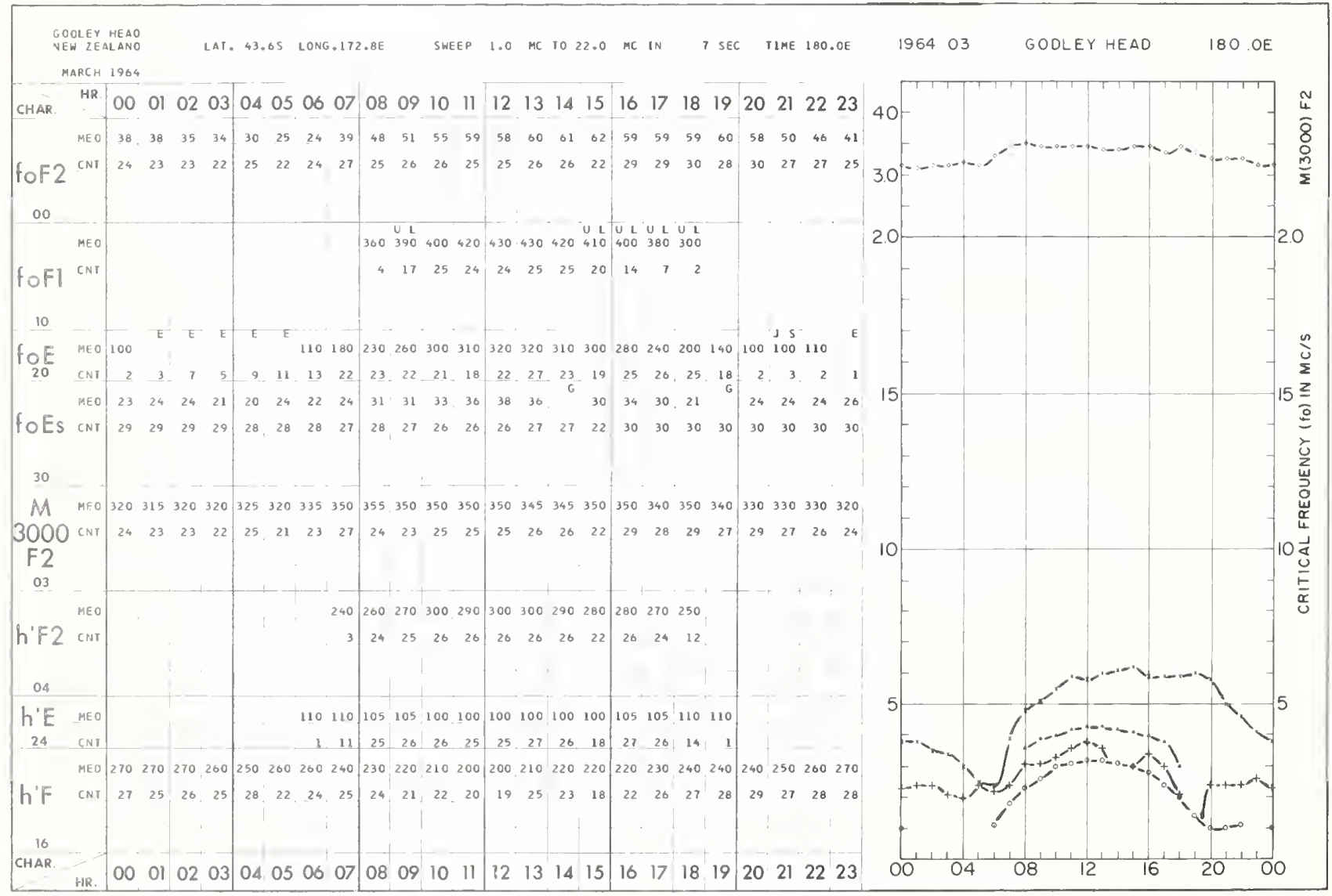



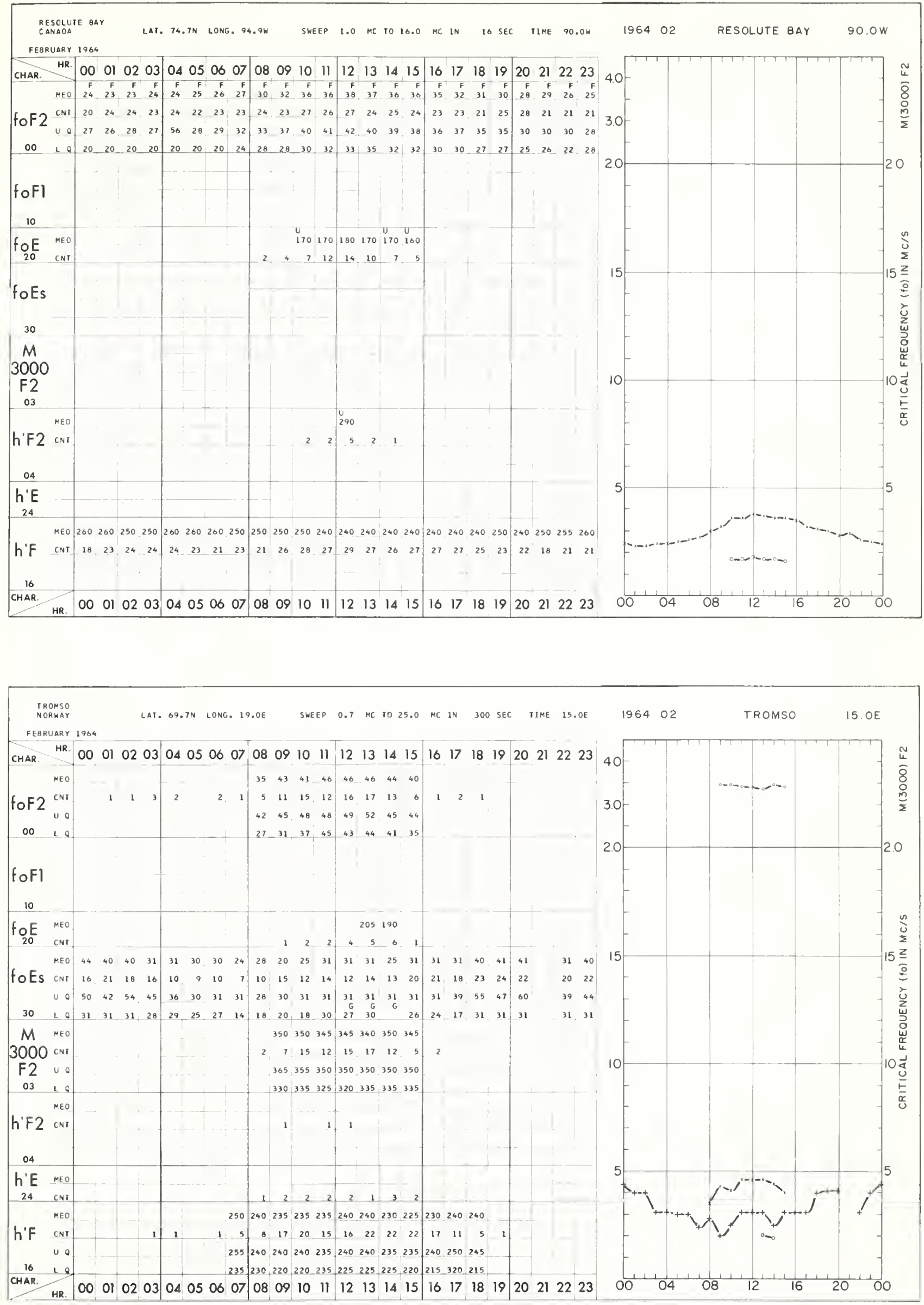

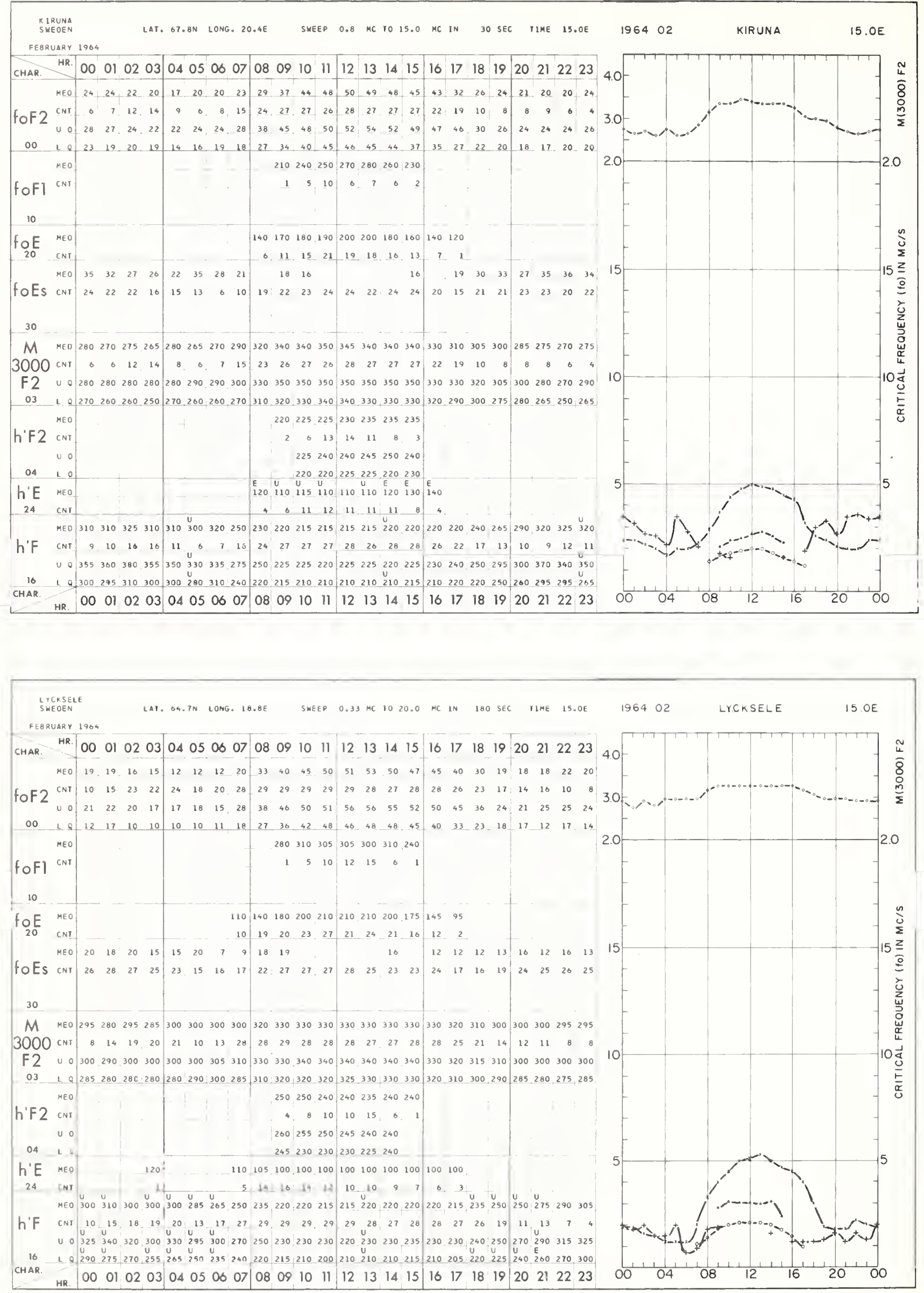


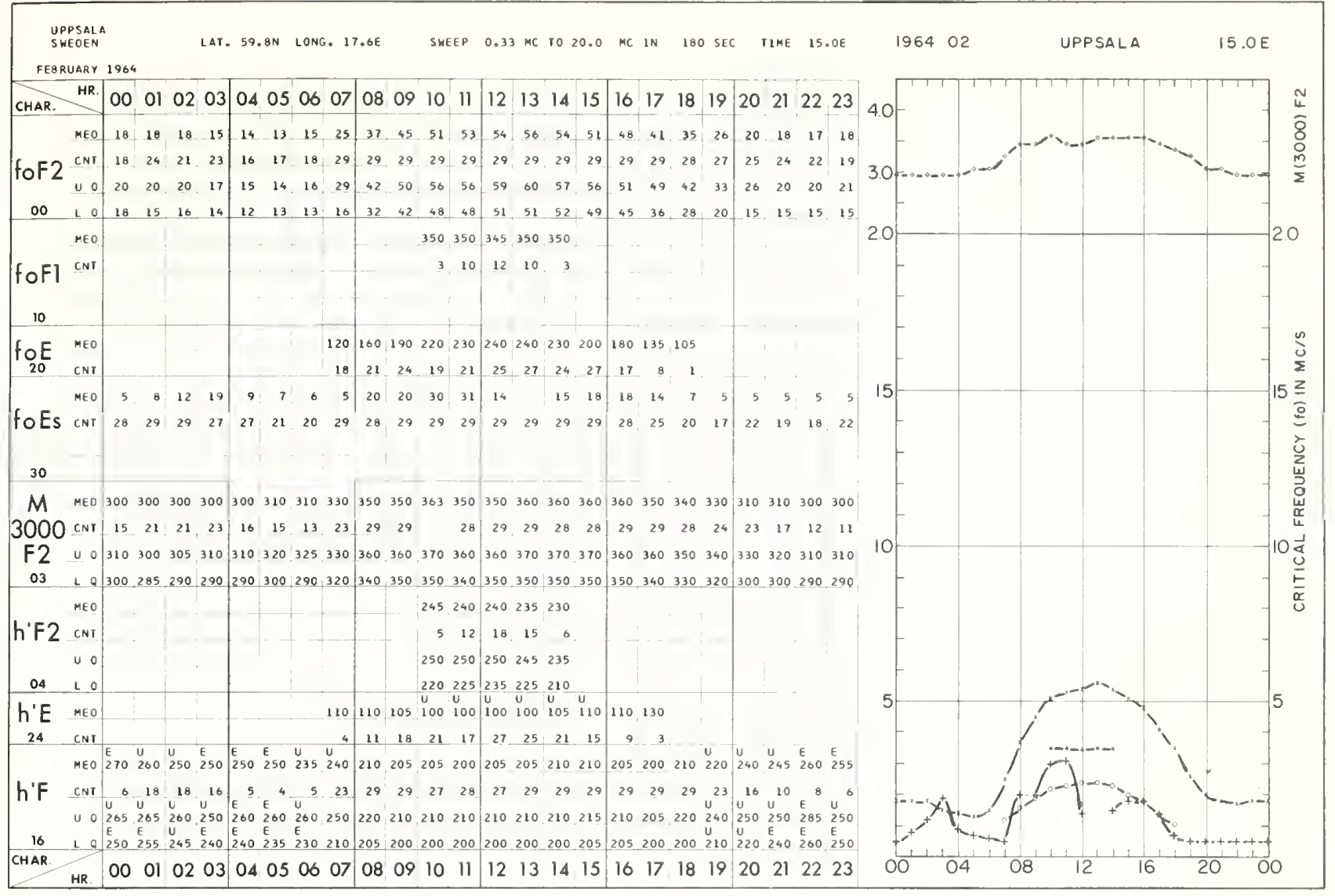

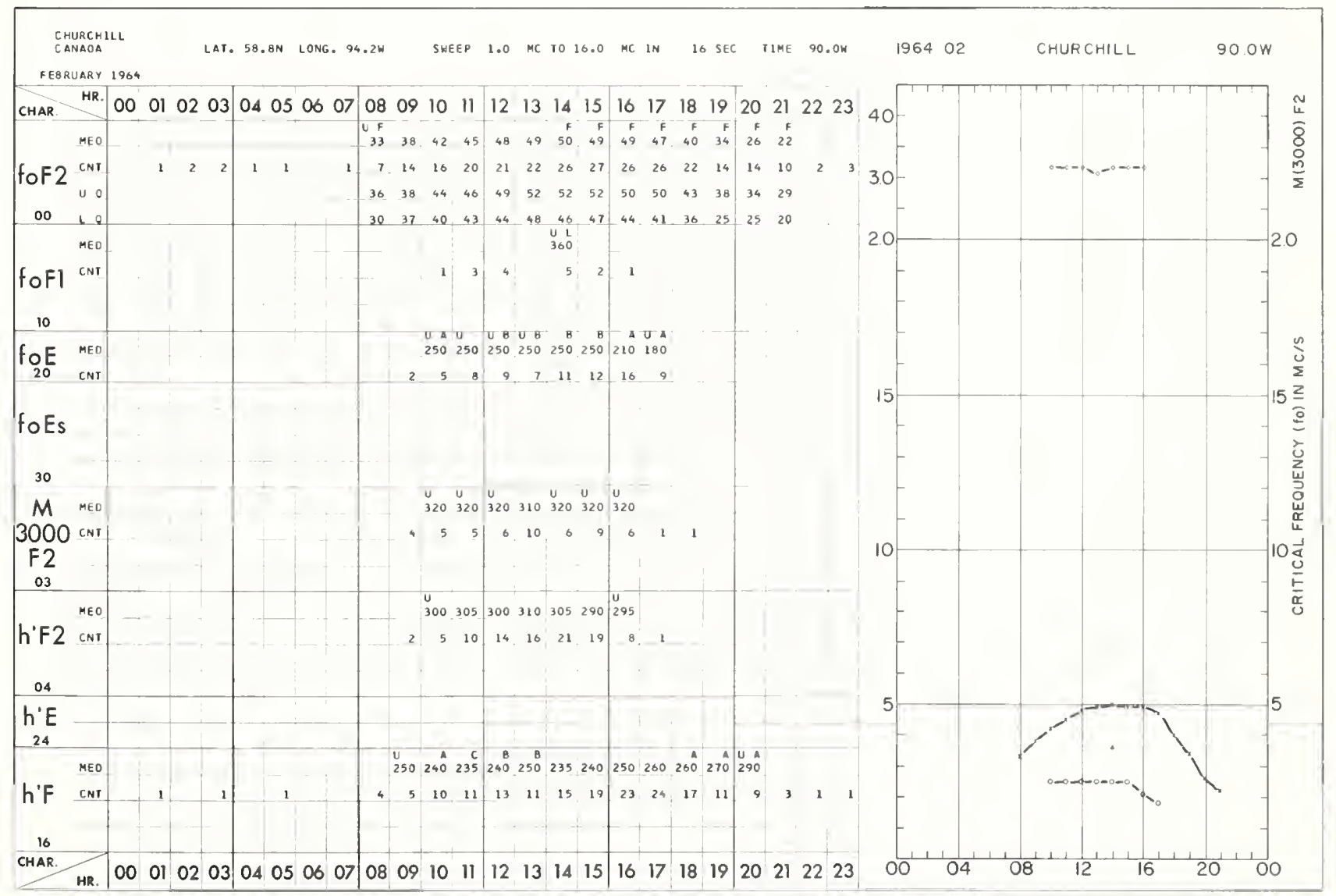



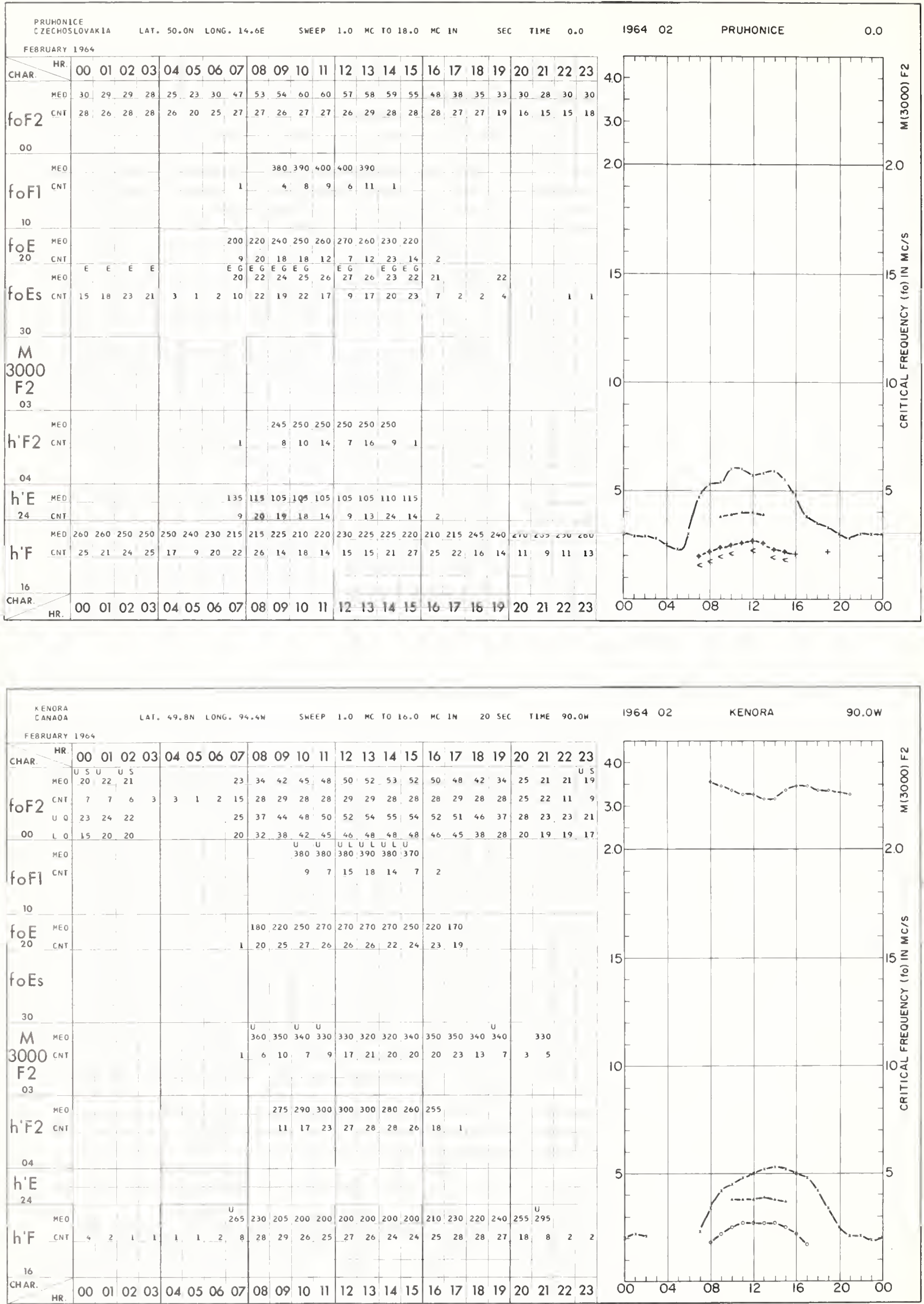
ST JOHNS
NEWFOUNOLANO

LAT. 47.6N LONG. 52.7

SHEEP 1.0 MC TO 16.0 MC IN 20 SEC TIME $60.0 \mathrm{~W}$

196402

ST JOHNS

$60.0 w$

FEBRUARY 1964

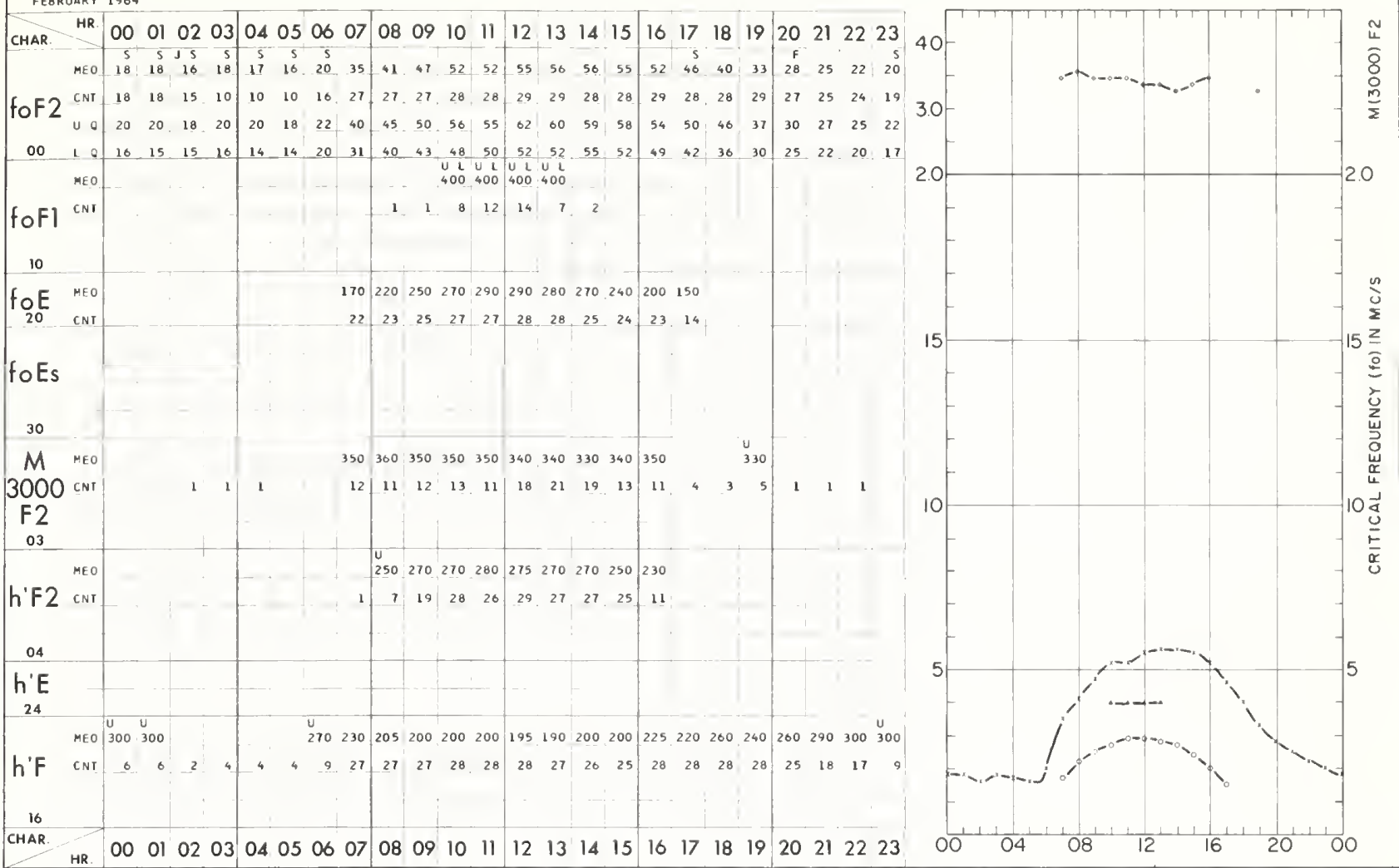

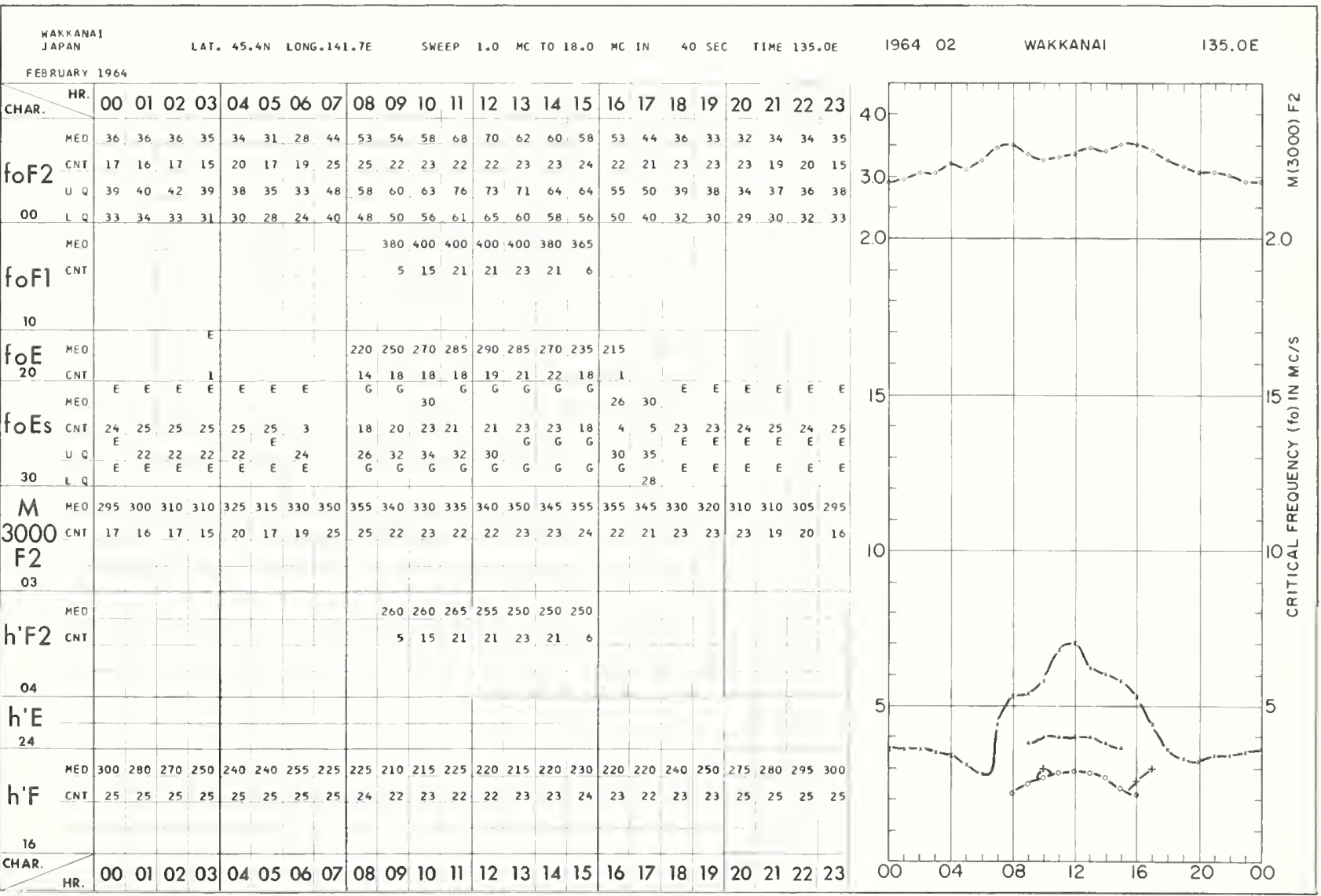



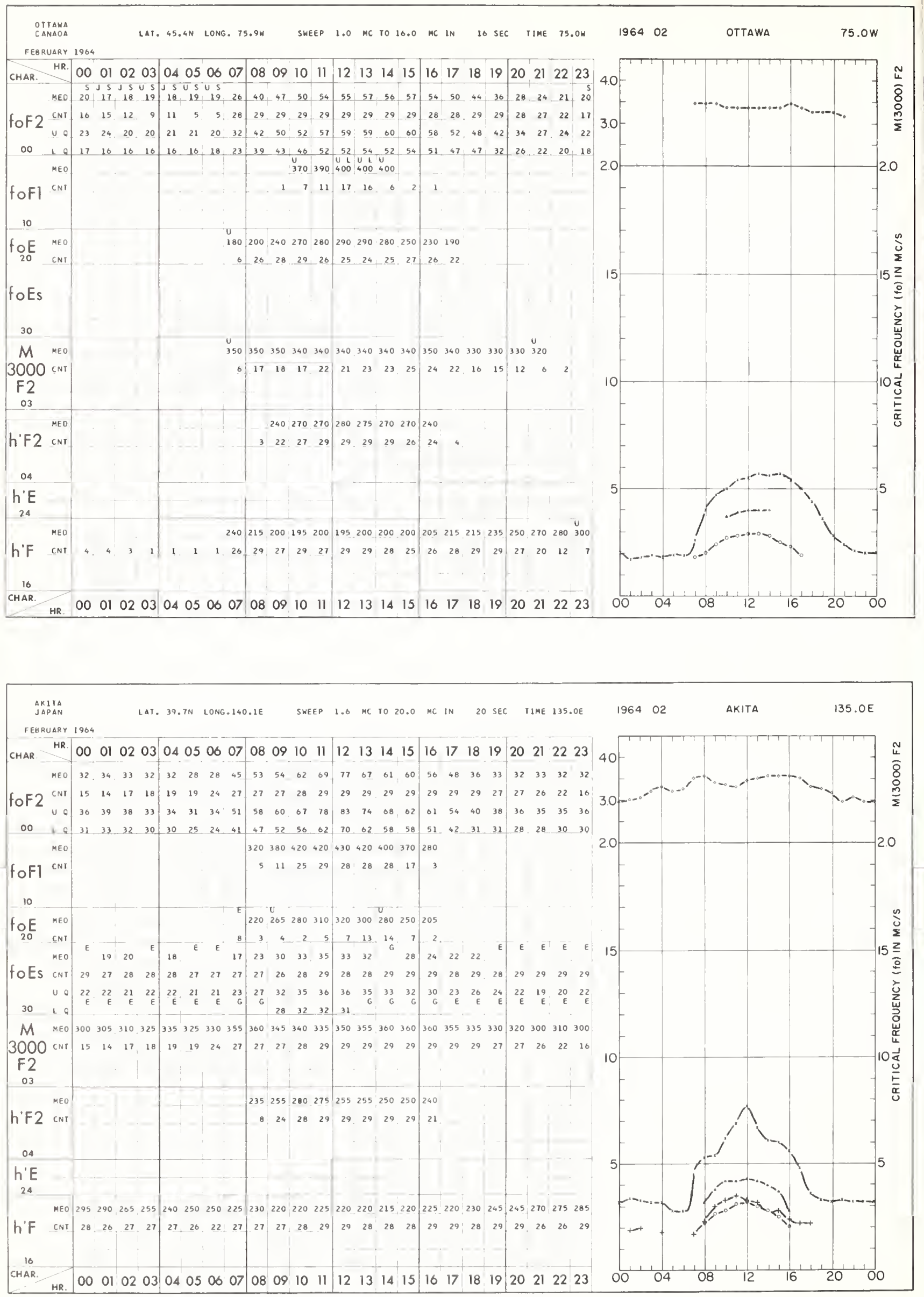


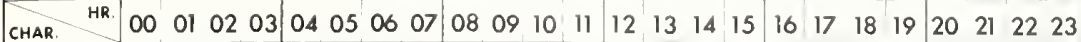
\begin{tabular}{l|llll|llll|lllllllllllllllll} 
MEO & 32 & 32 & 32 & 32 & 31 & 30 & 26 & 30 & 51 & 56 & 61 & 63 & 63 & 68 & 61 & 61 & 60 & 54 & 45 & 36 & 35 & 34 & 32 & 32
\end{tabular} foF2 \begin{tabular}{l|llll|lllll|llll|l|lllll|lllllllll}
$\mathrm{CNT}$ & 24 & 25 & 26 & 23 & 25 & 23 & 24 & 26 & 24 & 25 & 27 & 27 & 25 & 27 & 27 & 27 & 26 & 23 & 27 & 24 & 26 & 23 & 24 & 22
\end{tabular}

\begin{tabular}{lll|llll|lll|l|lll|l|l|l|llll|lllllllll} 
& 0 & 0 & 34 & 34 & 34 & 34 & 32 & 32 & 30 & 35 & 56 & 60 & 60 & 67 & 68 & 72 & 68 & 66 & 68 & 60 & 52 & 38 & 38 & 37 & 36 & 35 \\
00 & 1 & 9 & 30 & 29 & 30 & 30 & 28 & 28 & 25 & 27 & 48 & 52 & 56 & 56 & 59 & 61 & 58 & 55 & 57 & 51 & 41 & 32 & 32 & 31 & 31 & 30
\end{tabular}

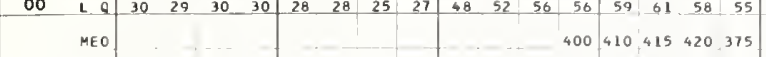

foFl CNT

\begin{tabular}{l|llll}
3 & 5 & 6 & 3 & 1
\end{tabular}

$410,455,420,430$

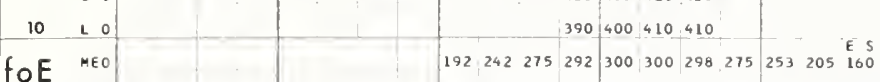

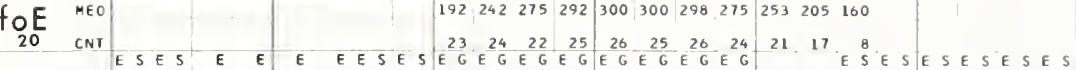
MEO ${ }_{16}$ I $_{16}$ E E

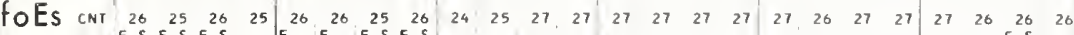

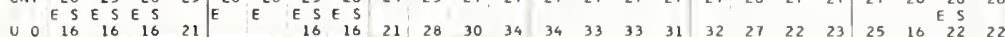

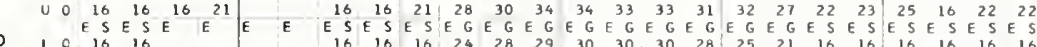

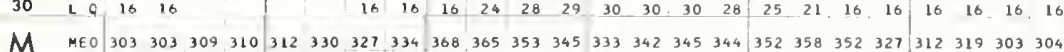
\begin{tabular}{|l|llll|lll|l|l|llll|lllll|llllllll}
3000 & $\mathrm{CNT}$ & 24 & 25 & 26 & 23 & 25 & 23 & 24 & 26 & 24 & 25 & 27 & 27 & 25 & 27 & 27 & 27 & 26 & 23 & 27 & 24 & 26 & 23 & 24 & 22
\end{tabular}

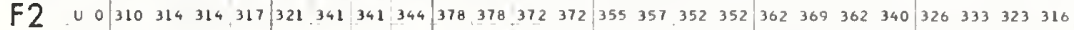

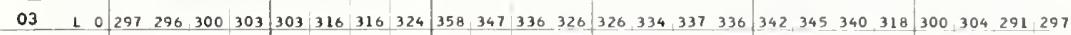

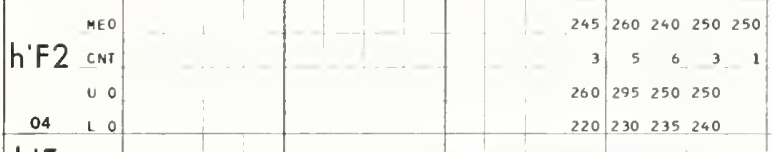

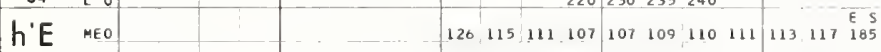

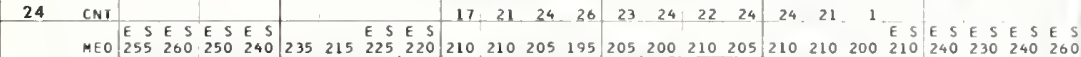

\begin{tabular}{ll|llll|llll|llllllll|llllllll} 
h'F & CNT & 26 & 25 & 26 & 25 & 26 & 26 & 25 & 26 & 24 & 25 & 27 & 27 & 27 & 27 & 27 & 27 & 27 & 26 & 27 & 27 & 27 & 26 & 26 & 26
\end{tabular}

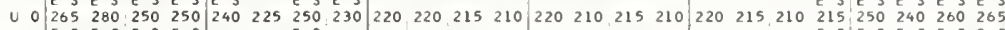

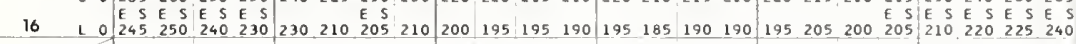
\begin{tabular}{|l|llll|llll|lllll|llll|llllllll} 
CHAR. HR & 00 & 01 & 02 & 03 & 04 & 05 & 06 & 07 & 08 & 09 & 10 & 11 & 12 & 13 & 14 & 15 & 16 & 17 & 18 & 19 & 20 & 21 & 22 & 23
\end{tabular}

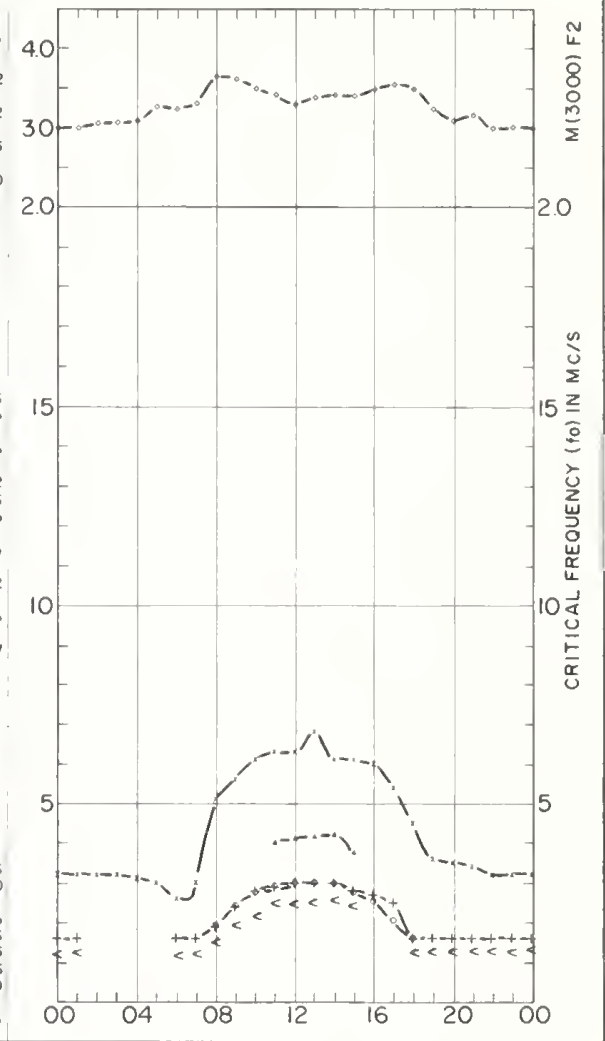

KOKUQUNJI
JAPAN LAT. $35.7 \mathrm{~N}$ LONG.139.5E

SWEEP 1.0 MC TO 20.0 MC IN 20 SEC TIME 135.0 .0

FESRUARY 1964

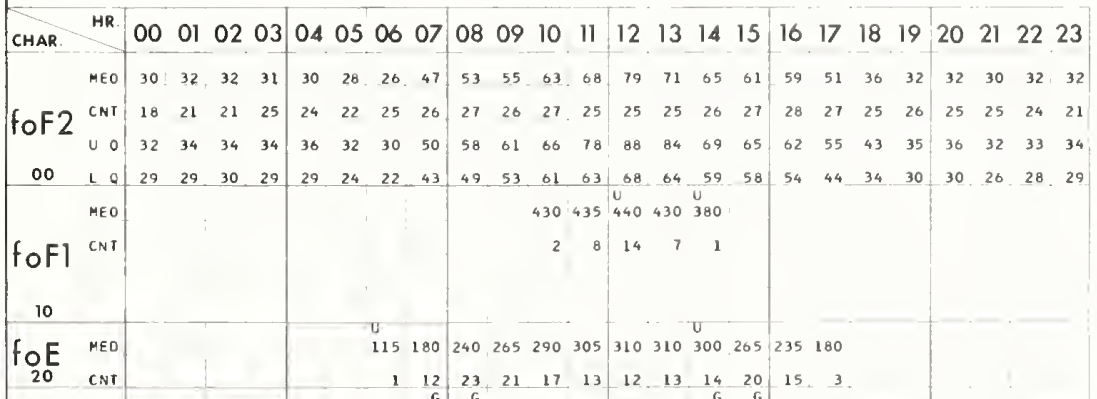
\begin{tabular}{llllllllll|lllllllllllllllll}
$M E O$ & 21 & 23 & 23 & 22 & 24 & 21 & 19 & $G$ & $G$ & 29 & 34 & 34 & 34 & 31 & $G$ & $G$ & 24 & 24 & 21 & 22 & 24 & 20 & 23 & 23
\end{tabular} \begin{tabular}{llllll|llllllllllllll|llllllllll} 
foES CNI & 15 & 14 & 15 & 14 & 13 & 13 & 7 & 15 & 25 & 25 & 25 & 23 & 25 & 23 & 25 & 25 & 25 & 18 & 16 & 18 & 16 & 15 & 16 & 17
\end{tabular} 30

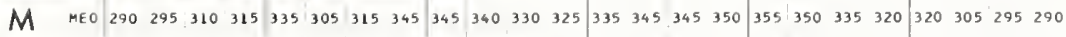
\begin{tabular}{|l|llll|l|l|ll|l|l|lll|llll|llll|llll}
3000 & $\mathrm{CNI}$ & 18 & 21 & 21 & 25 & 24 & 22 & 25 & 26 & 27 & 26 & 27 & 25 & 25 & 25 & 26 & 27 & 28 & 27 & 25 & 26 & 25 & 25 & 24 & 21
\end{tabular}

$\mathrm{F} 2$
03

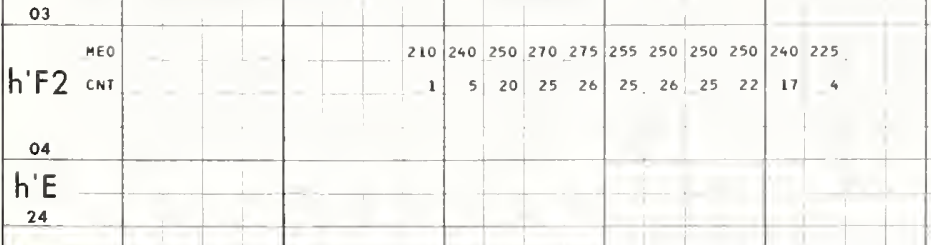

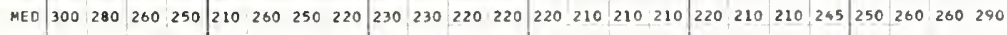

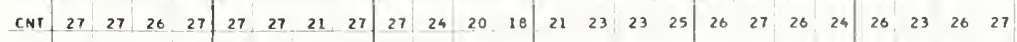
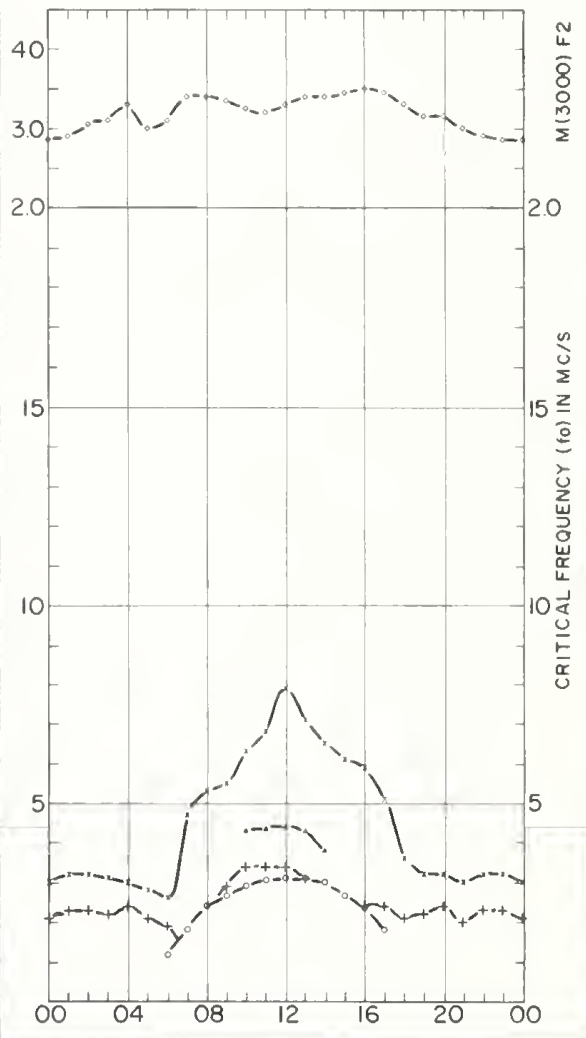


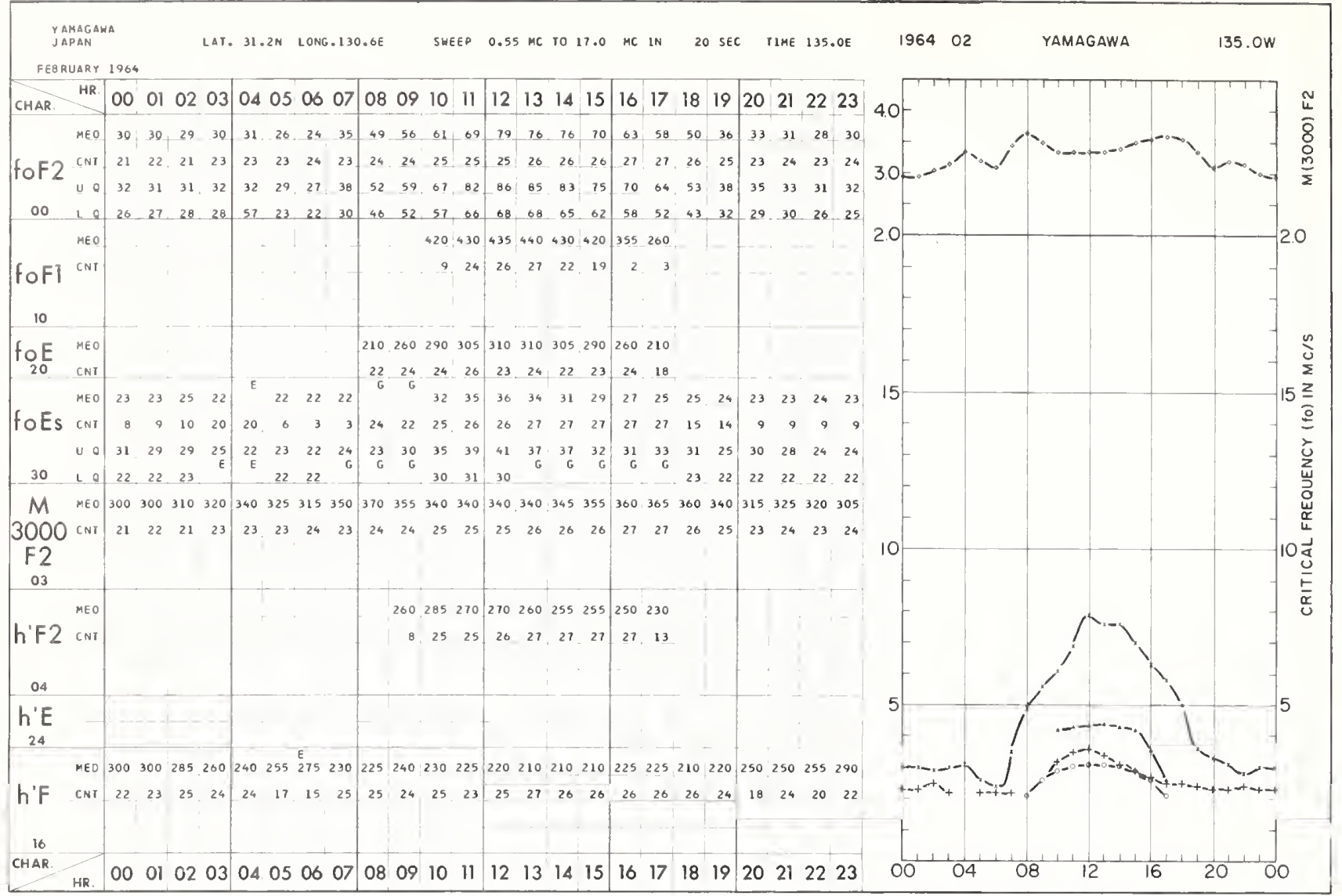

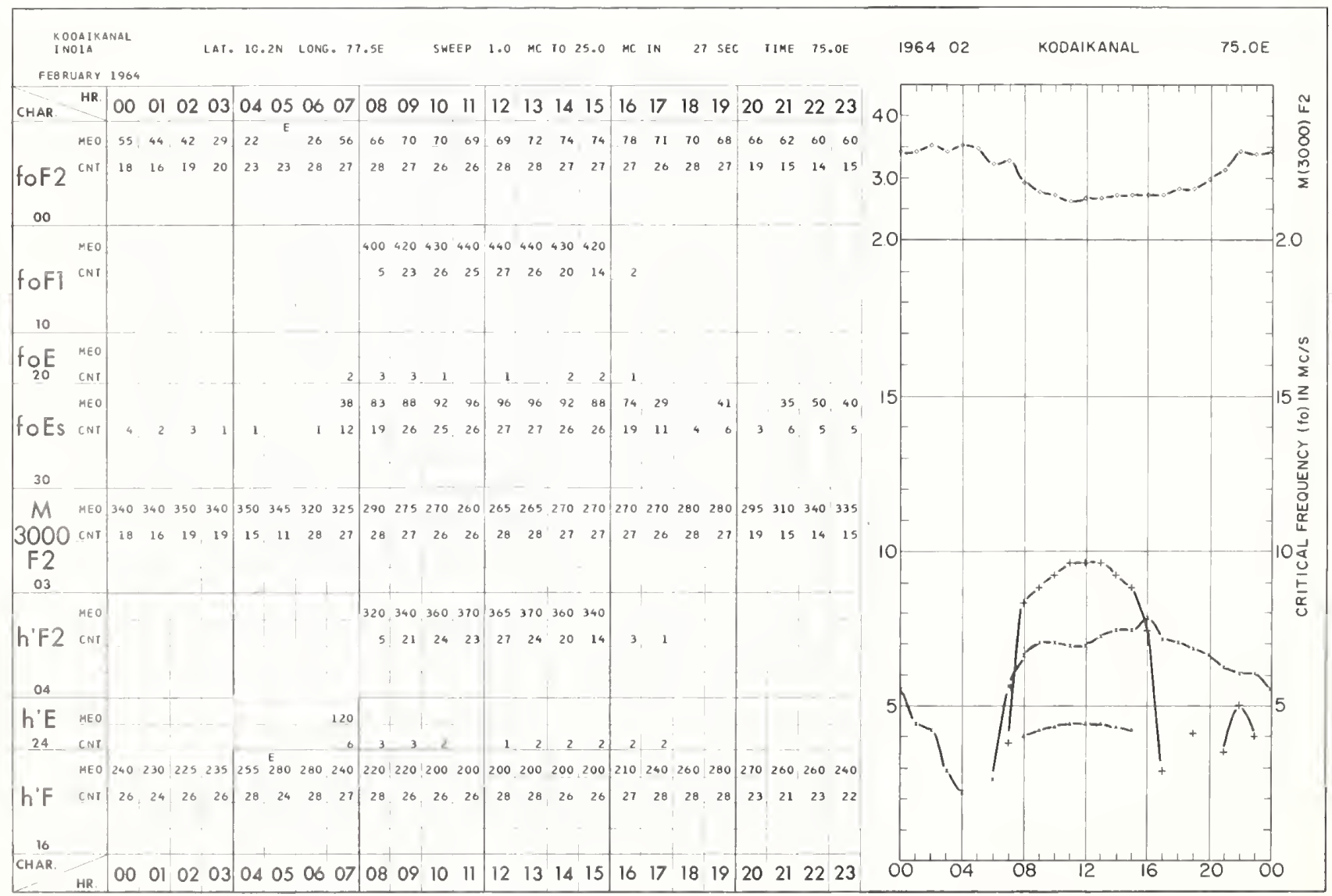




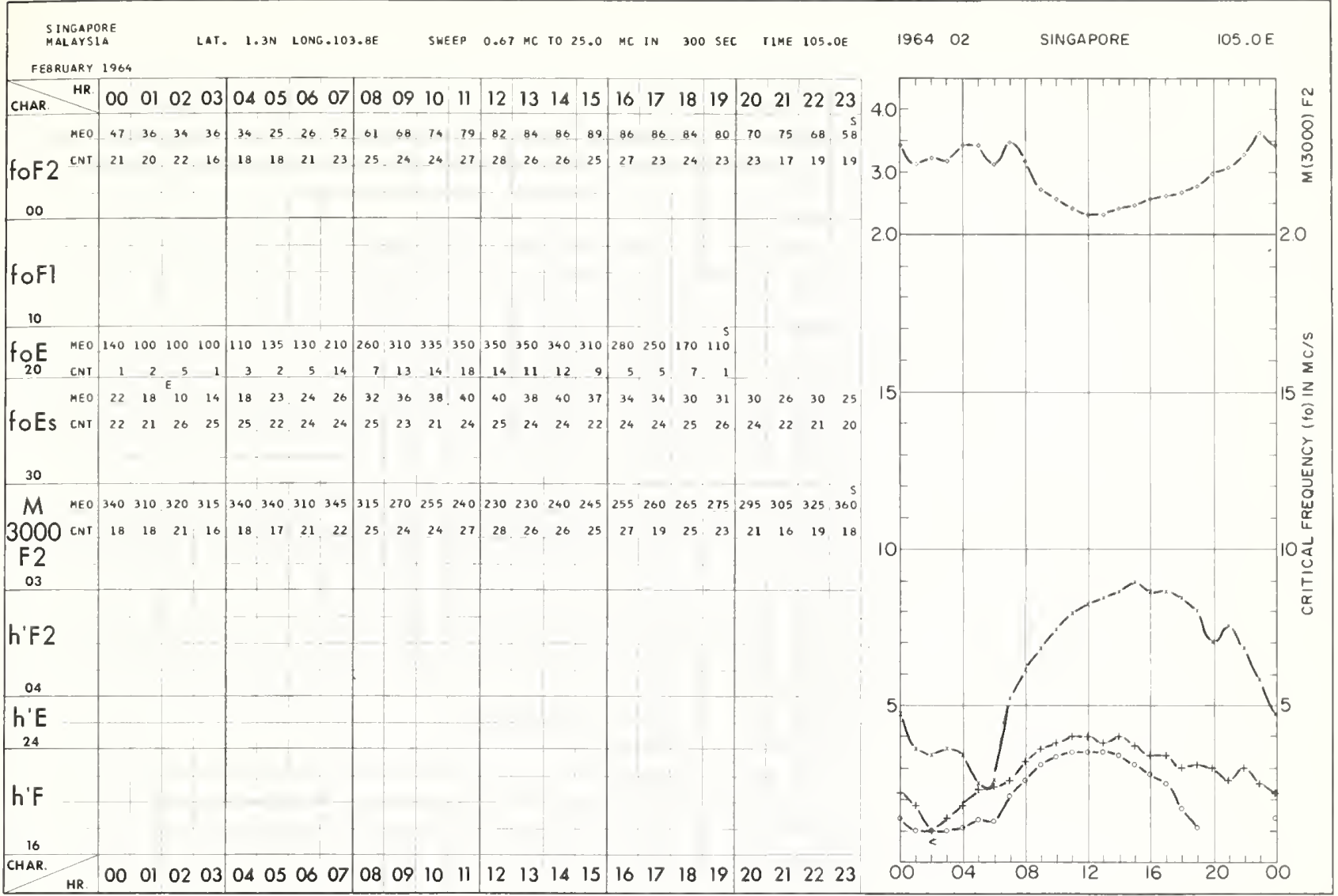

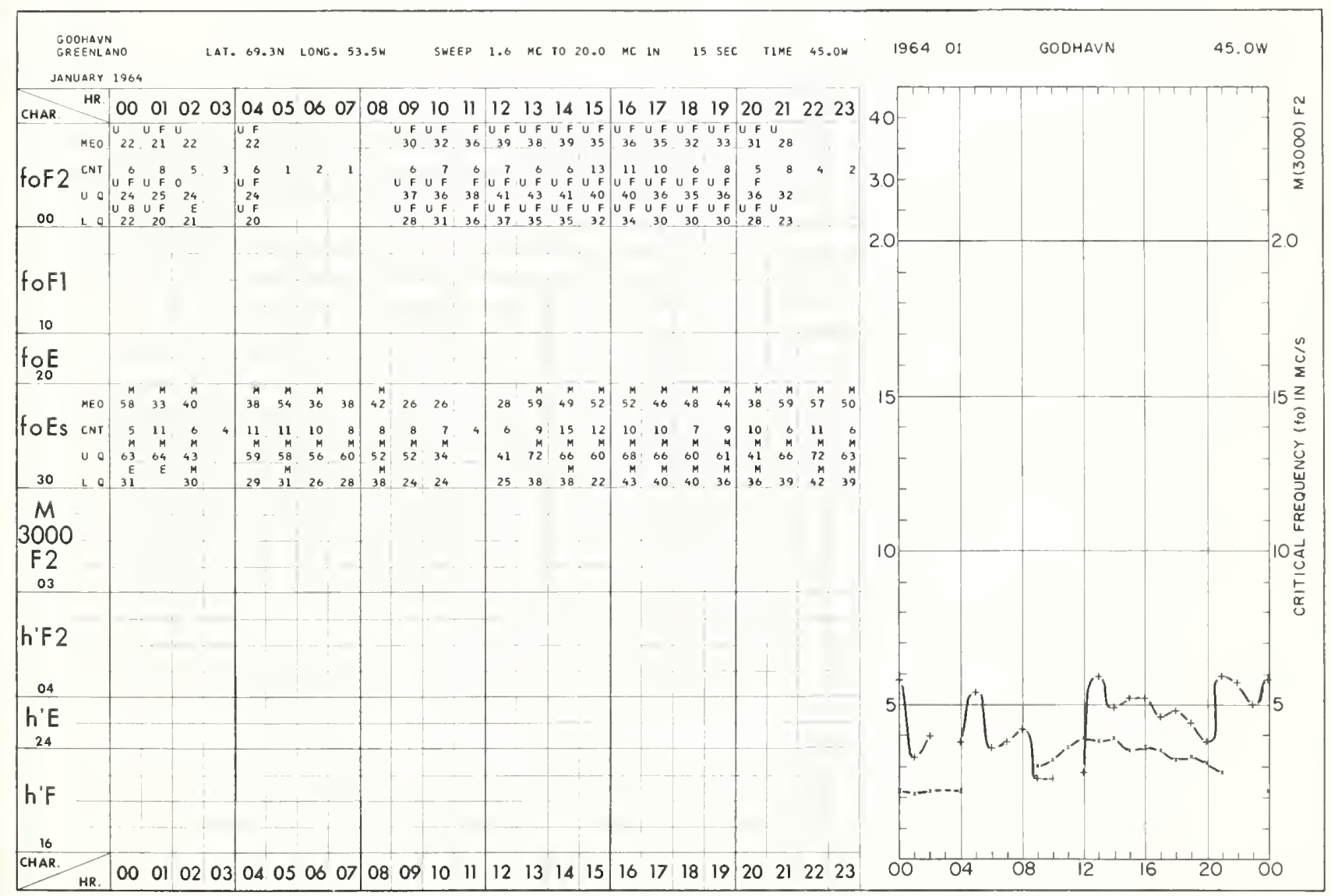




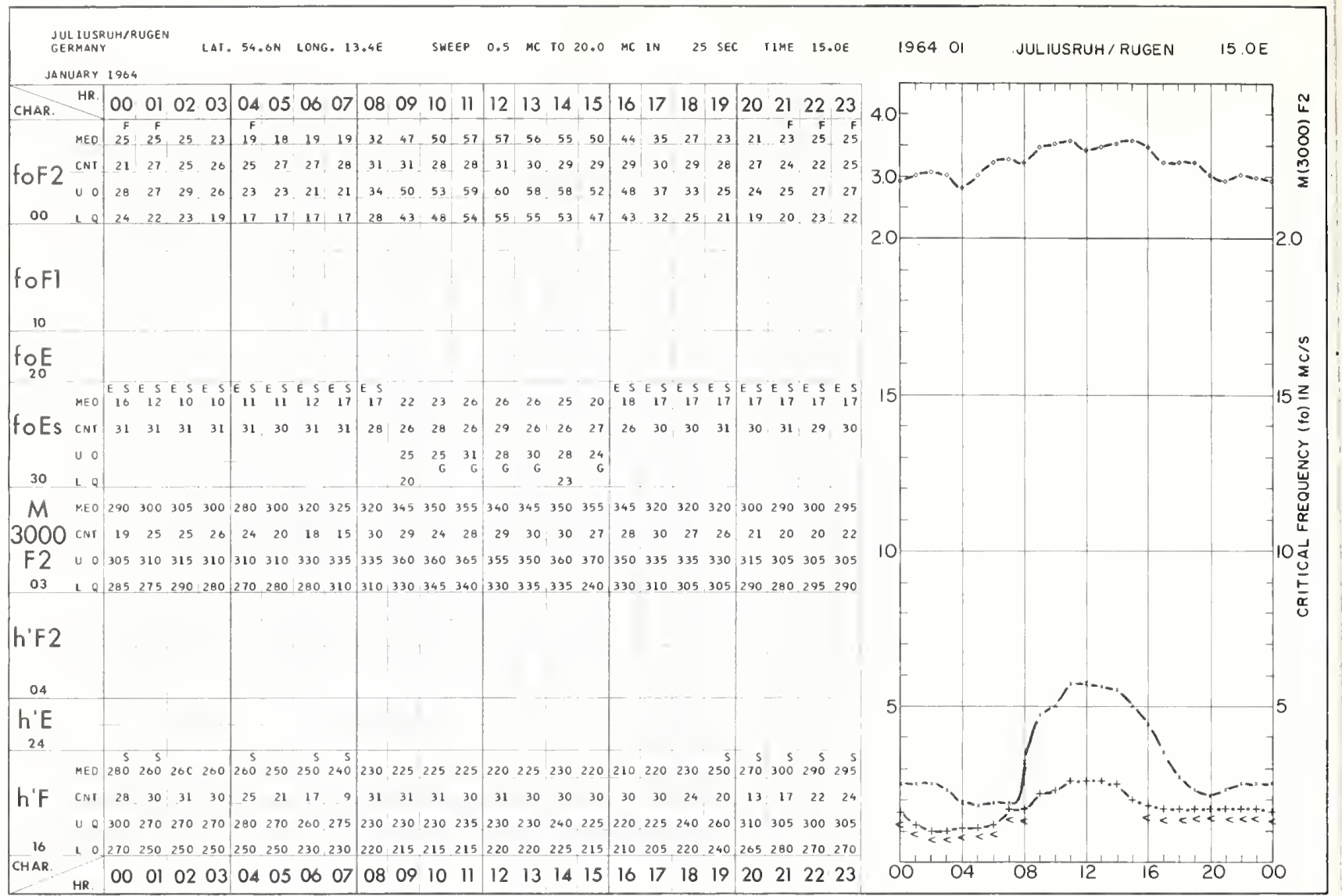

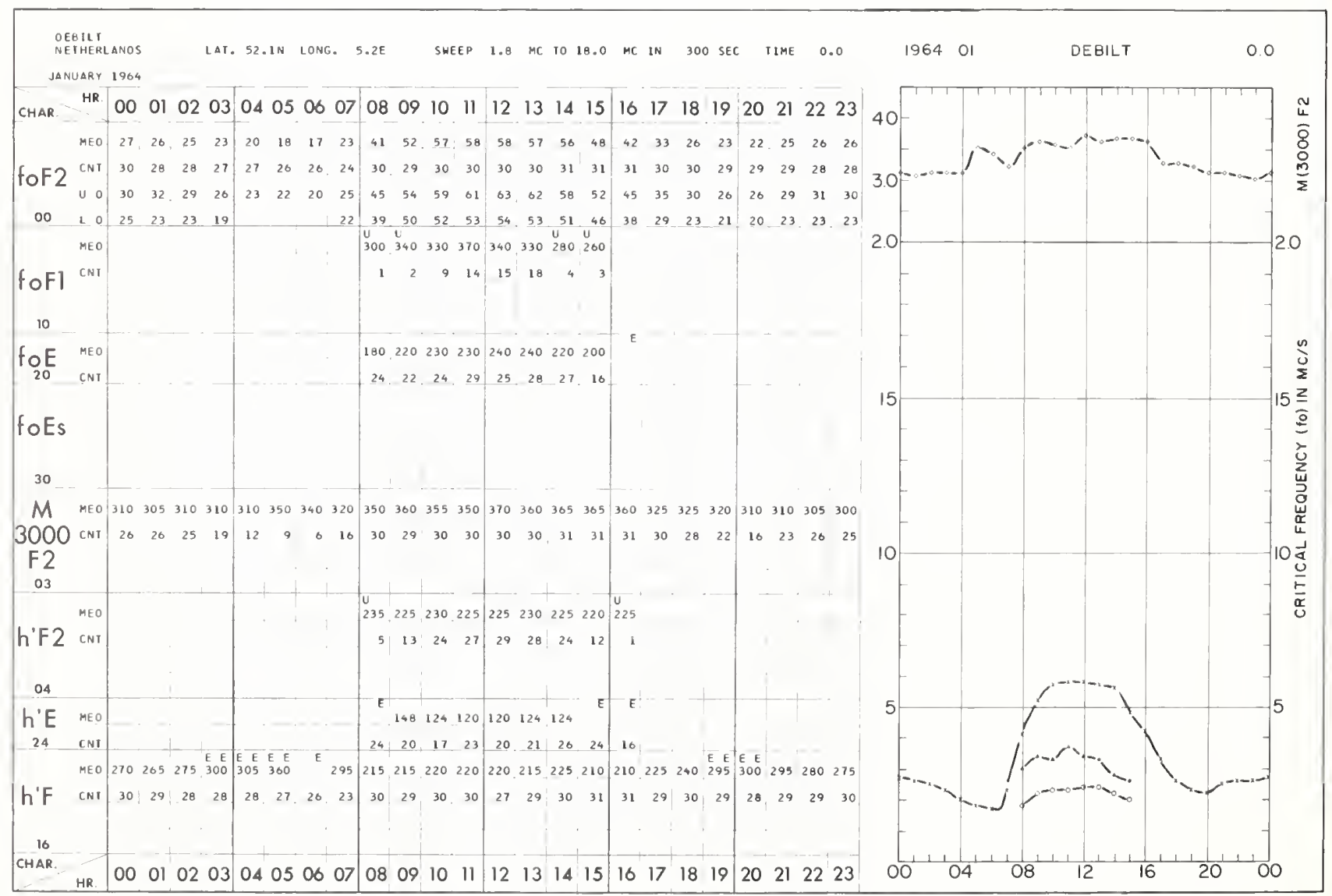


PRUHONICE

C2ECHOSLOVAK

LAT. 50. ON LONG. 14.6E

SWEEP 1.0 HC TO 18.0 MC IN

SEC TIME 0.0

1964 이

PRUHONICE

0.0

JANUARY 1964

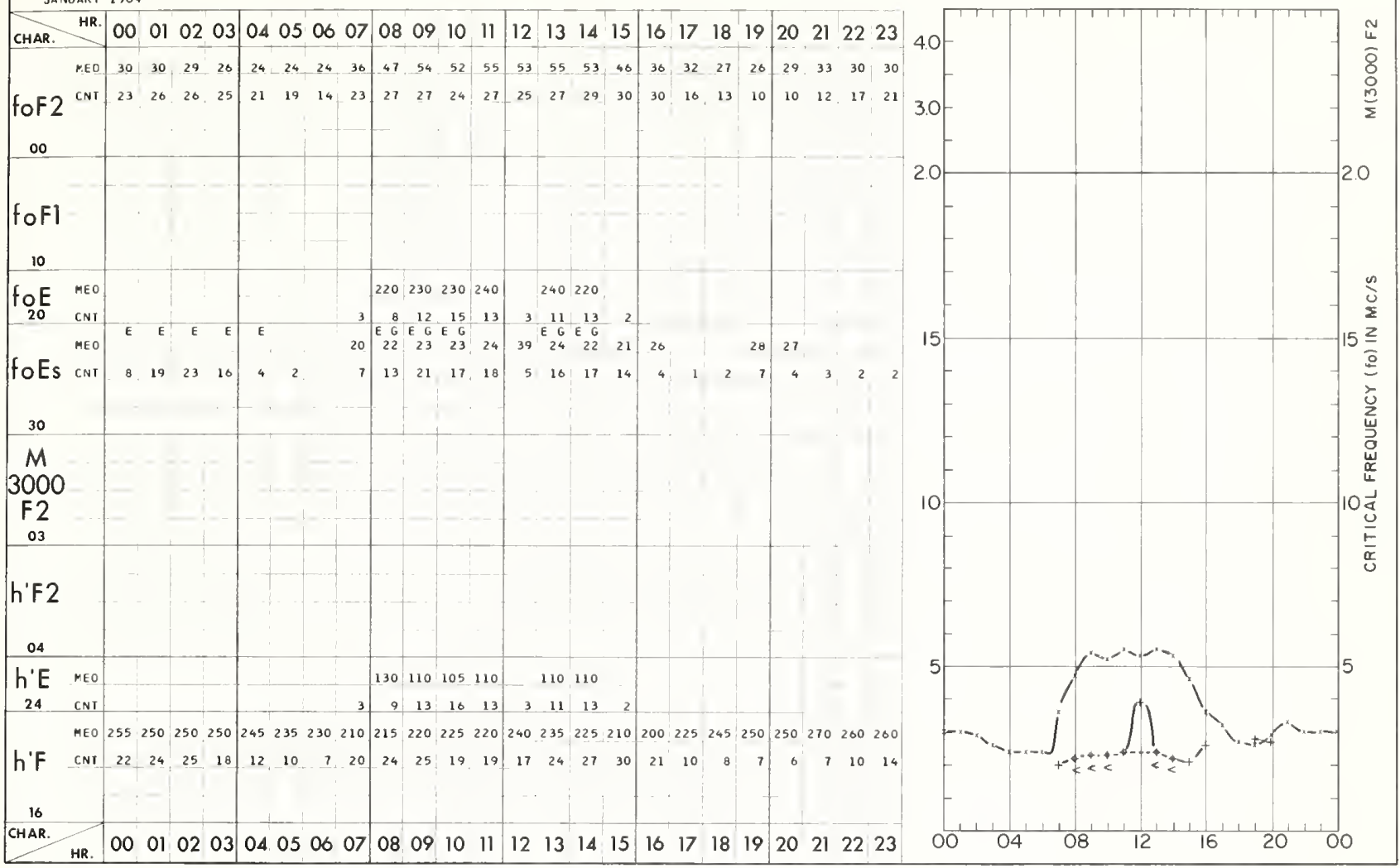

SOTIENS

SHITZERLANO

LAT. 46. 6N LONG. 6.7E

SHEEP 1.0 MC TO 25.0 MC IN 30 SEC TIME 15.0 .0E

19640

SOTTENS

$15 \mathrm{OE}$

JANUARY 1904

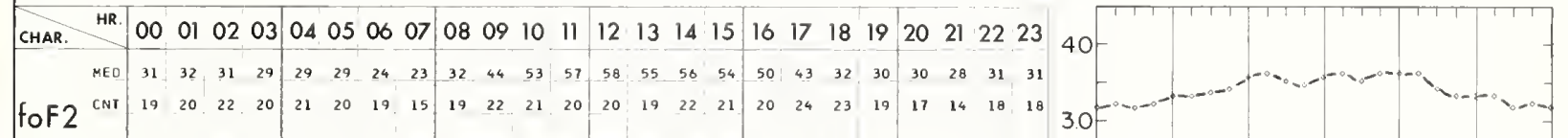

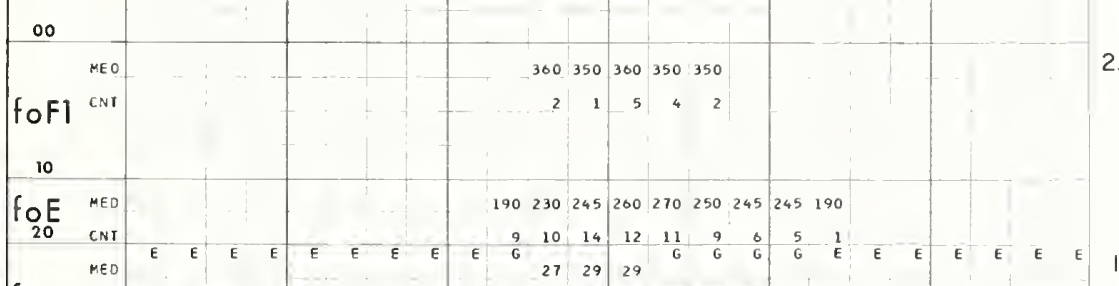

\begin{tabular}{|lllll|lllll|l|llll|ll|lll|l|lllllll} 
foEs CNT & 23 & 23 & 23 & 23 & 23 & 23 & 23 & 23 & 23 & 23 & 22 & 23 & 21 & 22 & 23 & 23 & 20 & 24 & 23 & 22 & 20 & 21 & 22 & 22
\end{tabular} 30

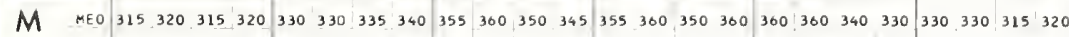

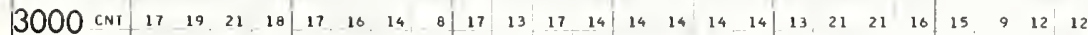

F2 h' ' $\begin{array}{lllll}330 & 225 & 250 & 230 & 250\end{array}$ 4 5. 4 .

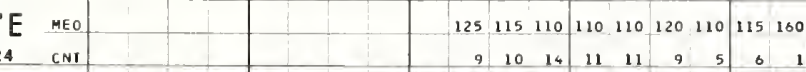

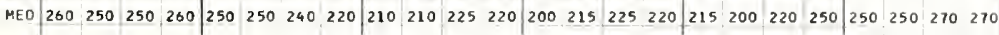
\begin{tabular}{ll|l|l|l|l|llll|l|l|ll|ll|ll|ll|l|lllll} 
h'F & CNT & 22 & 22 & 23 & 23 & 23 & 23 & 21 & 13 & 20 & 23 & 21 & 21 & 22 & 20 & 21 & 23 & 21 & 24 & 23 & 19 & 17 & 13 & 15 & 16
\end{tabular}

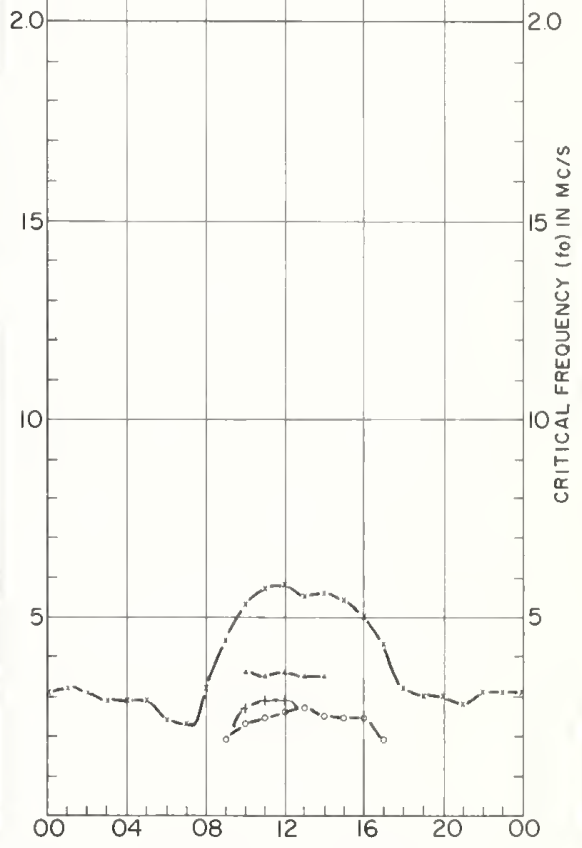



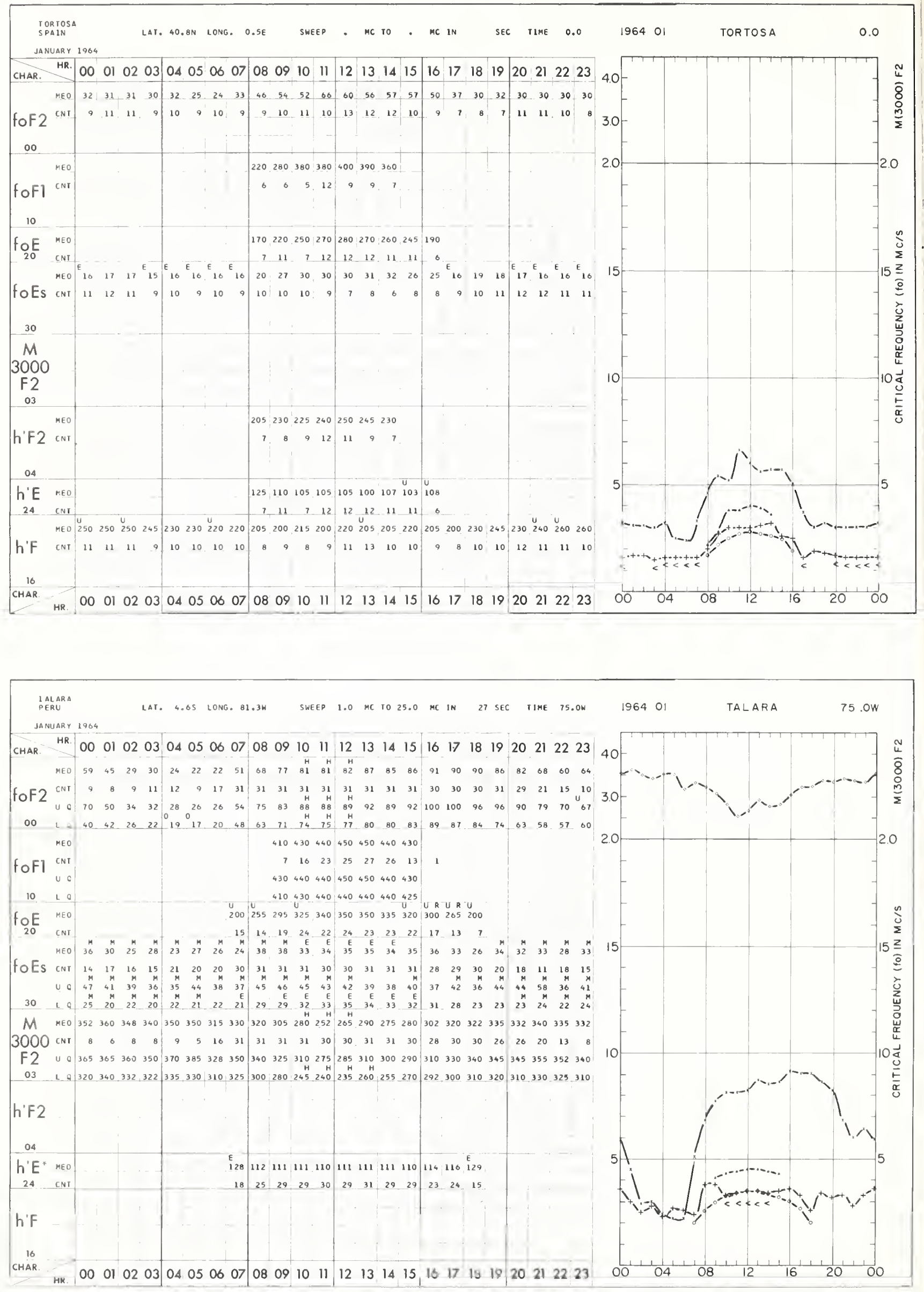
GOOHAVN

LAT. $69.3 N$ LONG. $53.5 \mathrm{~W}$

SWEEP 1.6 MC TO 20.0 MC IN 15 SEC TIME 45.0W

196312

GODHAVN

45 ow

OECEMBER 1963

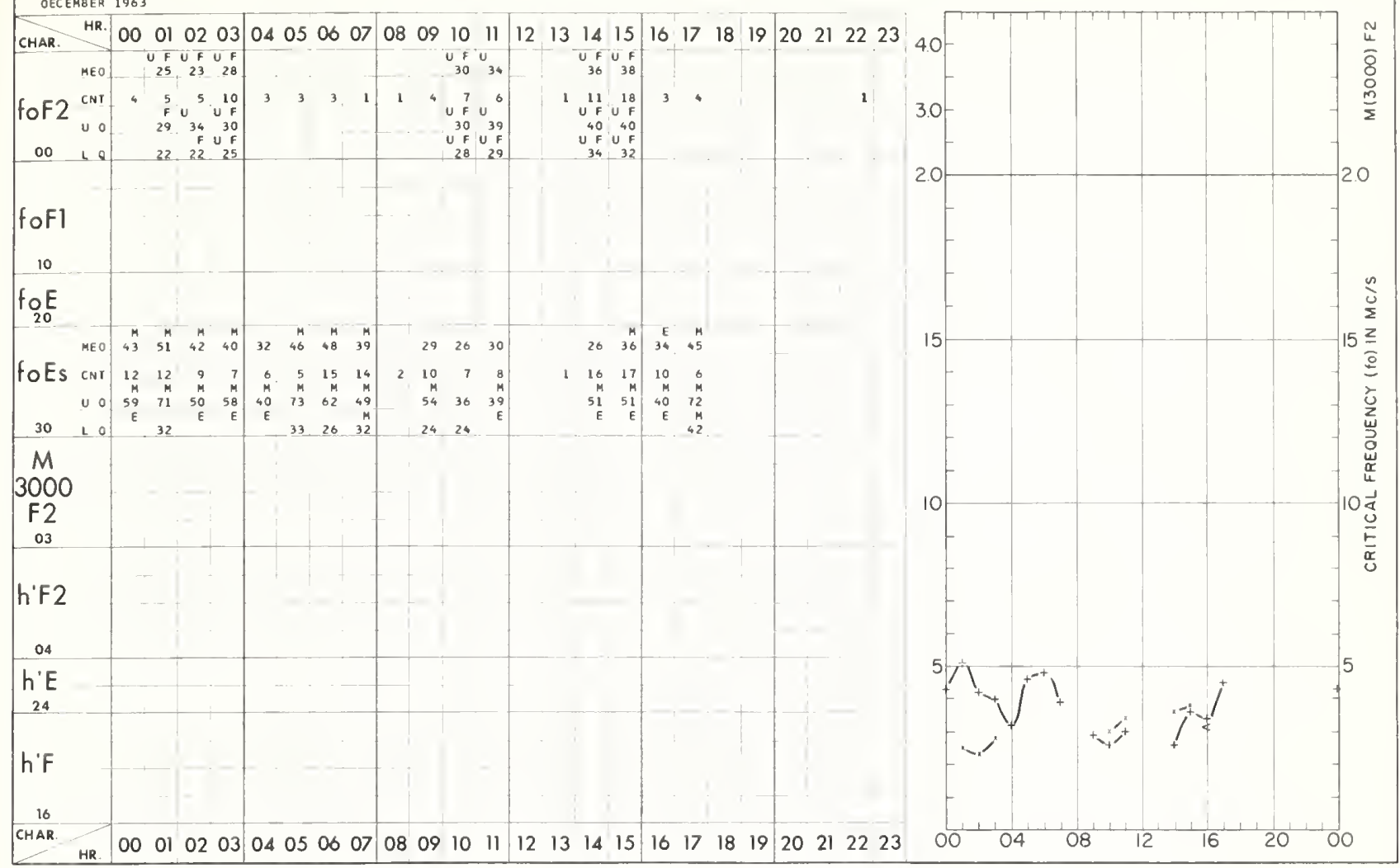

TALAR
PERU

LAT. 4.6S LONG. 8L.3W

SWEEP 1.0 MC TO 25.0 MC IN 27 SEC TIME $75.0 \mathrm{~N}$

196312

TALARA

$75.0 W$

OEC EMBER 1963

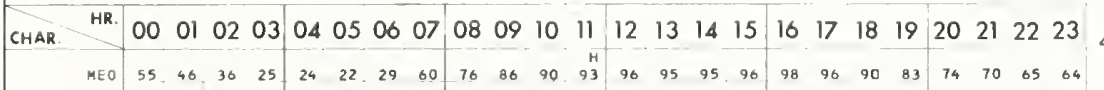

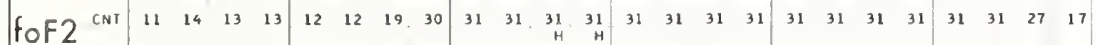

\begin{tabular}{ll|llll|llll|lllllllllllllllllll}
\hline & 0 & 0 & 66 & 56 & 40 & 38 & 32 & 26 & 33 & 62 & 81 & 90 & 95 & 95 & 102 & 105 & 100 & 101 & 102 & 100 & 100 & 93 & 84 & 78 & 73 & 68
\end{tabular}

\begin{tabular}{lllllll|llll|lll|l|llllllllllll}
00 & $L$ & 0 & 49 & 40 & 28 & 22 & 19 & 18 & 27 & 56 & 72 & 78 & 82 & 89 & 85 & 88 & 89 & 92 & 94 & 89 & 81 & 69 & 64 & 65 & 57 & 52
\end{tabular}

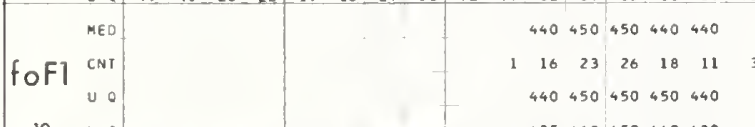

$10 \perp 0$

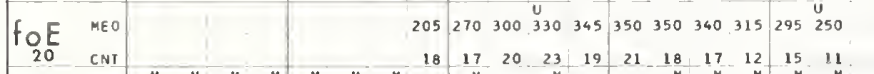

$435 \$ 40 \quad 450 \quad 440 \quad 430$

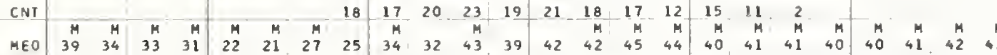

\begin{tabular}{llllll|lllllllll|lllll|llllllll}
\hline foES CNT & 27 & 25 & 21 & 17 & 16 & 17 & 13 & 31 & 31 & 31 & 30 & 31 & 31 & 31 & 31 & 31 & 31 & 31 & 30 & 28 & 28 & 27 & 22 & 26
\end{tabular}

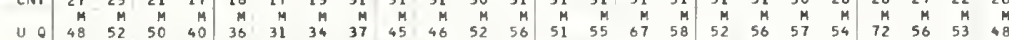

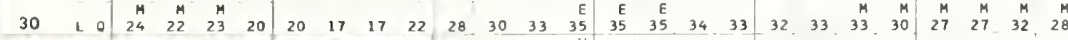

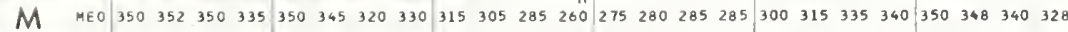

\begin{tabular}{ll|llll|llll|lllll|llll|llllllll}
$3000 \mathrm{CNT}$ & 10 & 10 & 11 & 11 & 8 & 8 & 18 & 29 & 31 & 31 & 30 & 31 & 27 & 30 & 28 & 31 & 30 & 28 & 31 & 31 & 28 & 30 & 23 & 12
\end{tabular}

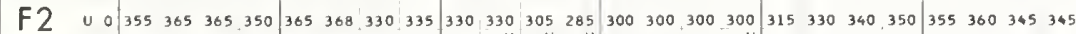

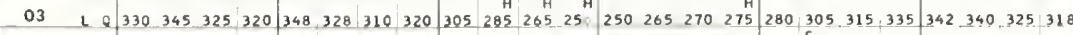

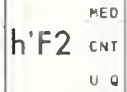

$04 \quad 0$

$h^{\prime} E^{*}$ MEO

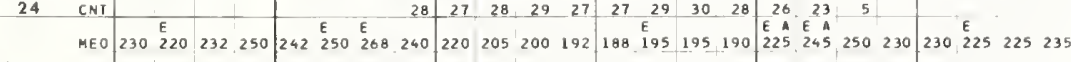

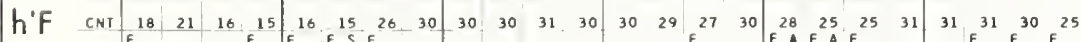

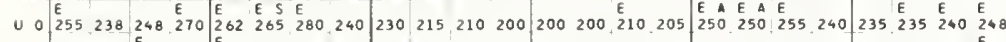

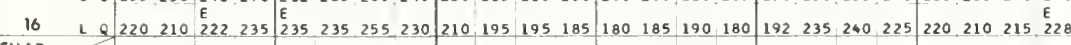

$\begin{array}{rlllllllllllllllllllllllll}\text { CHAR. } & 00 & 01 & 02 & 03 & 04 & 05 & 06 & 07 & 08 & 09 & 10 & 11 & 12 & 13 & 14 & 15 & 16 & 17 & 18 & 19 & 20 & 21 & 22 & 23\end{array}$

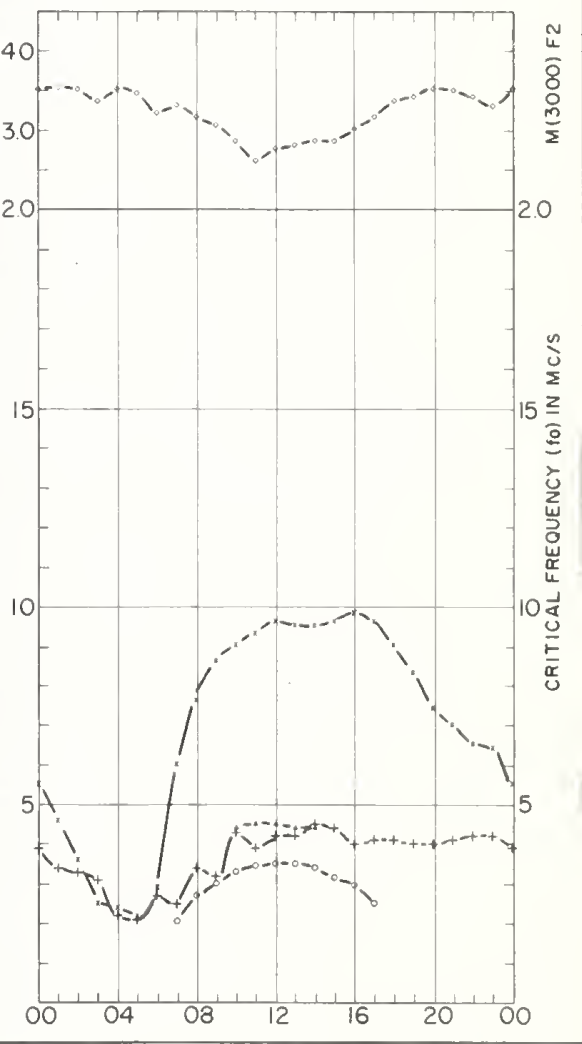



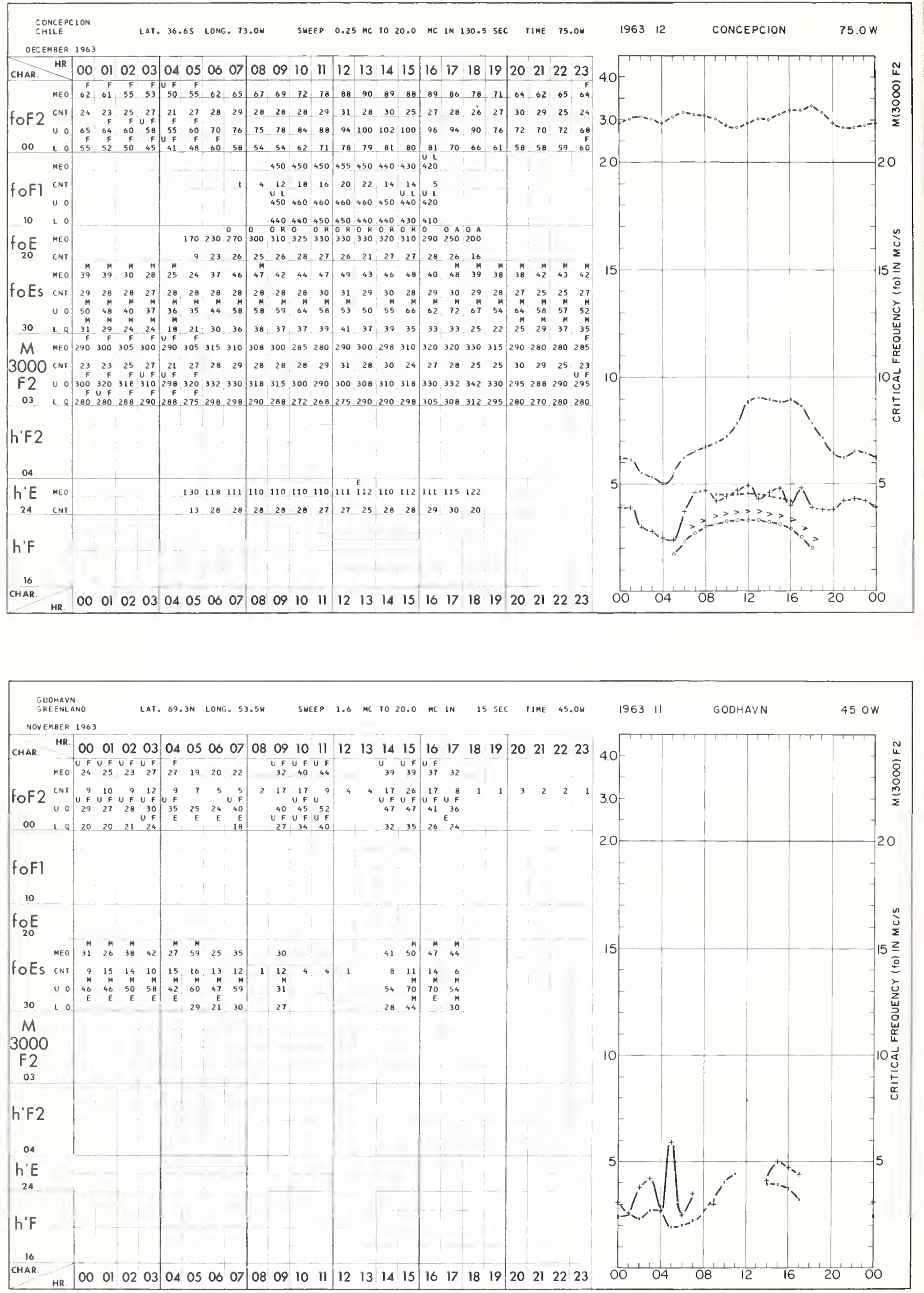


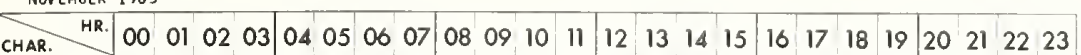

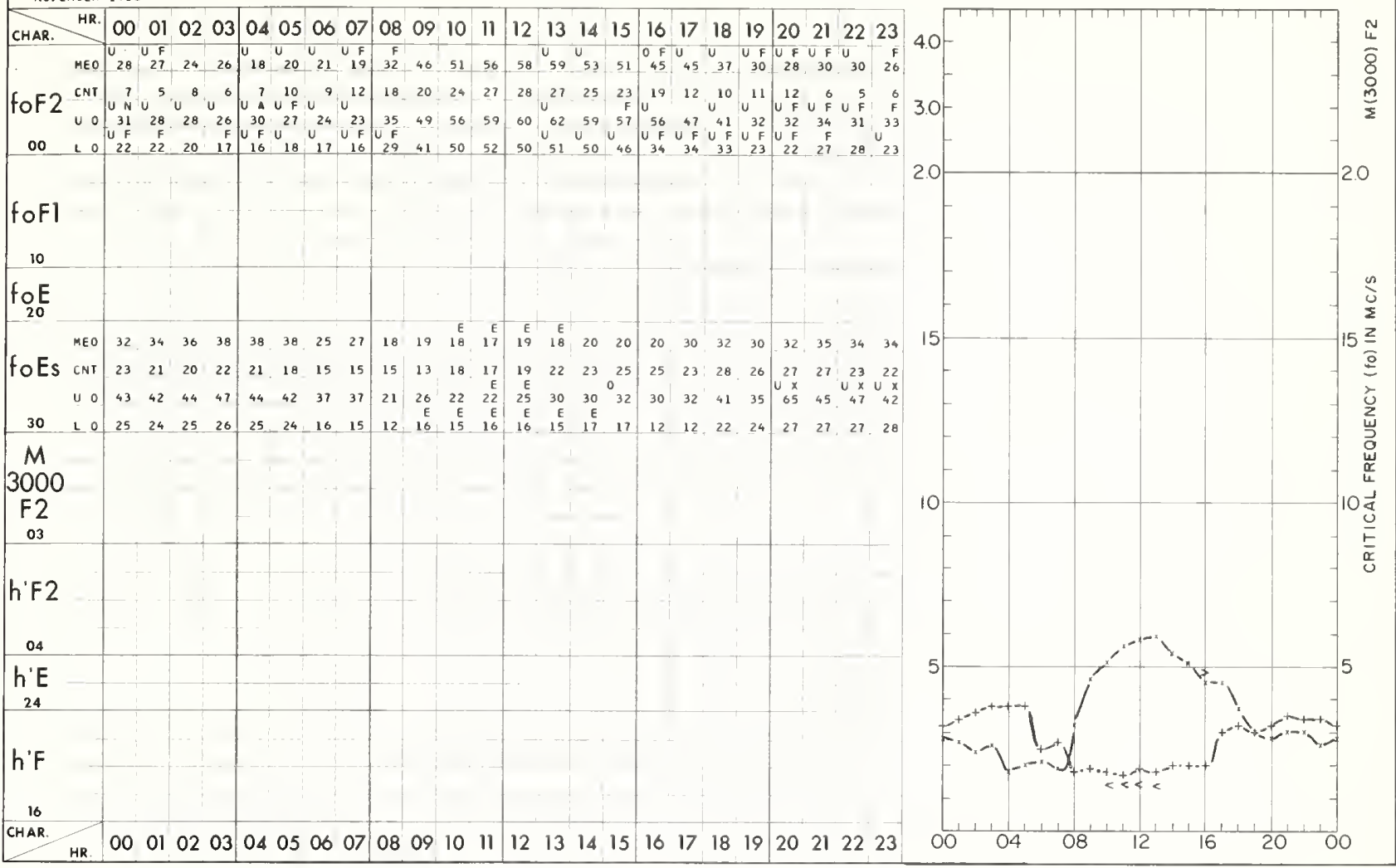

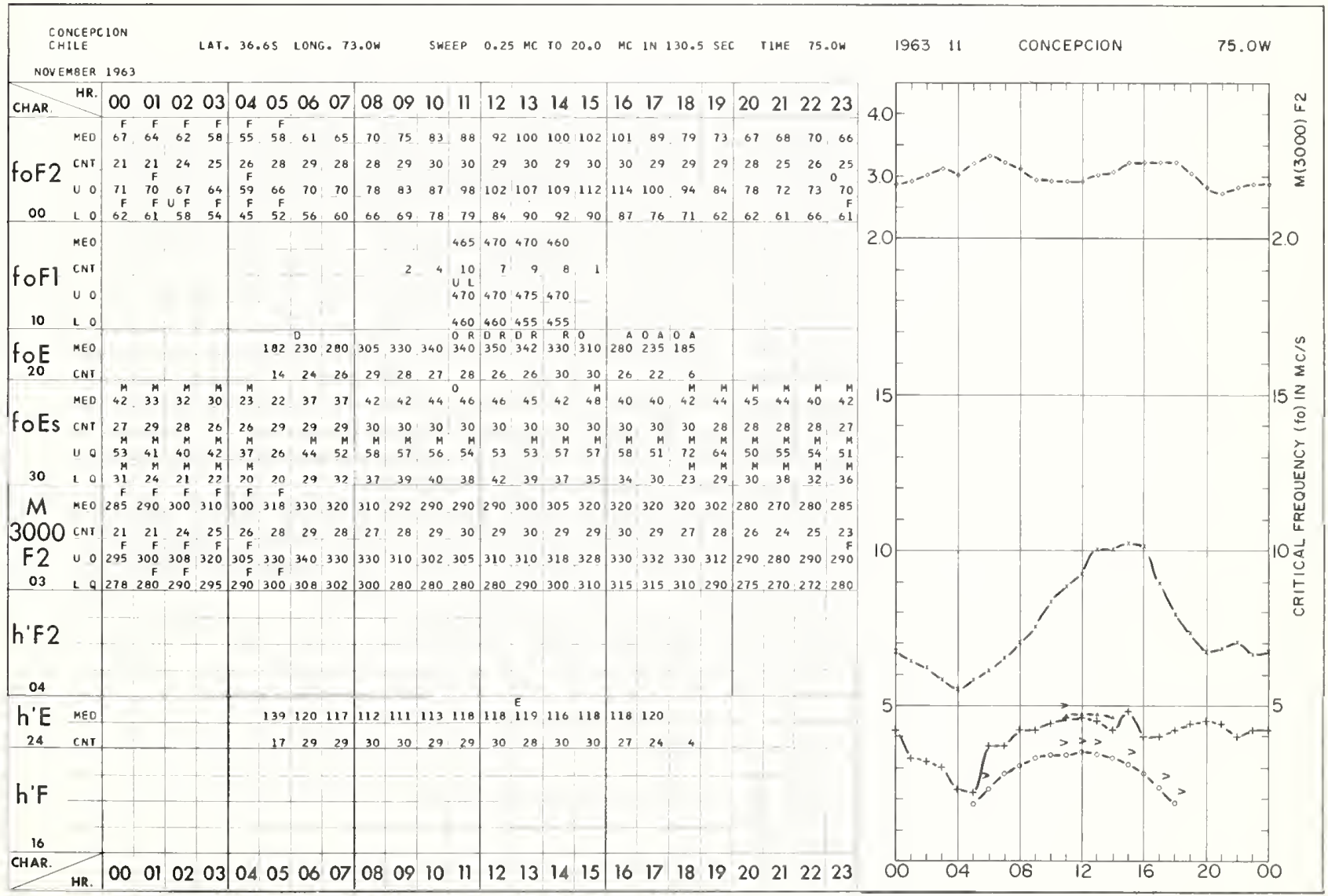




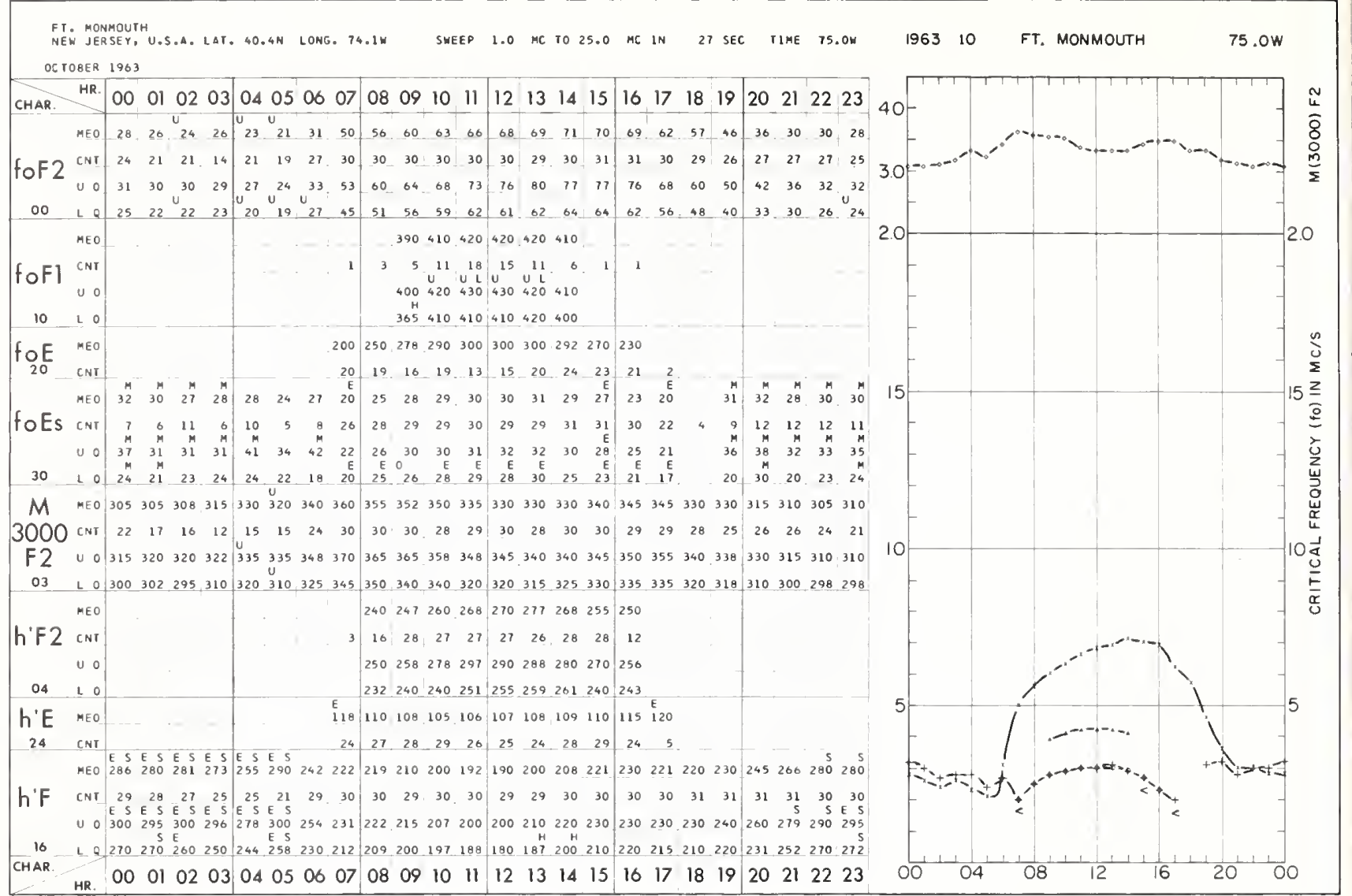

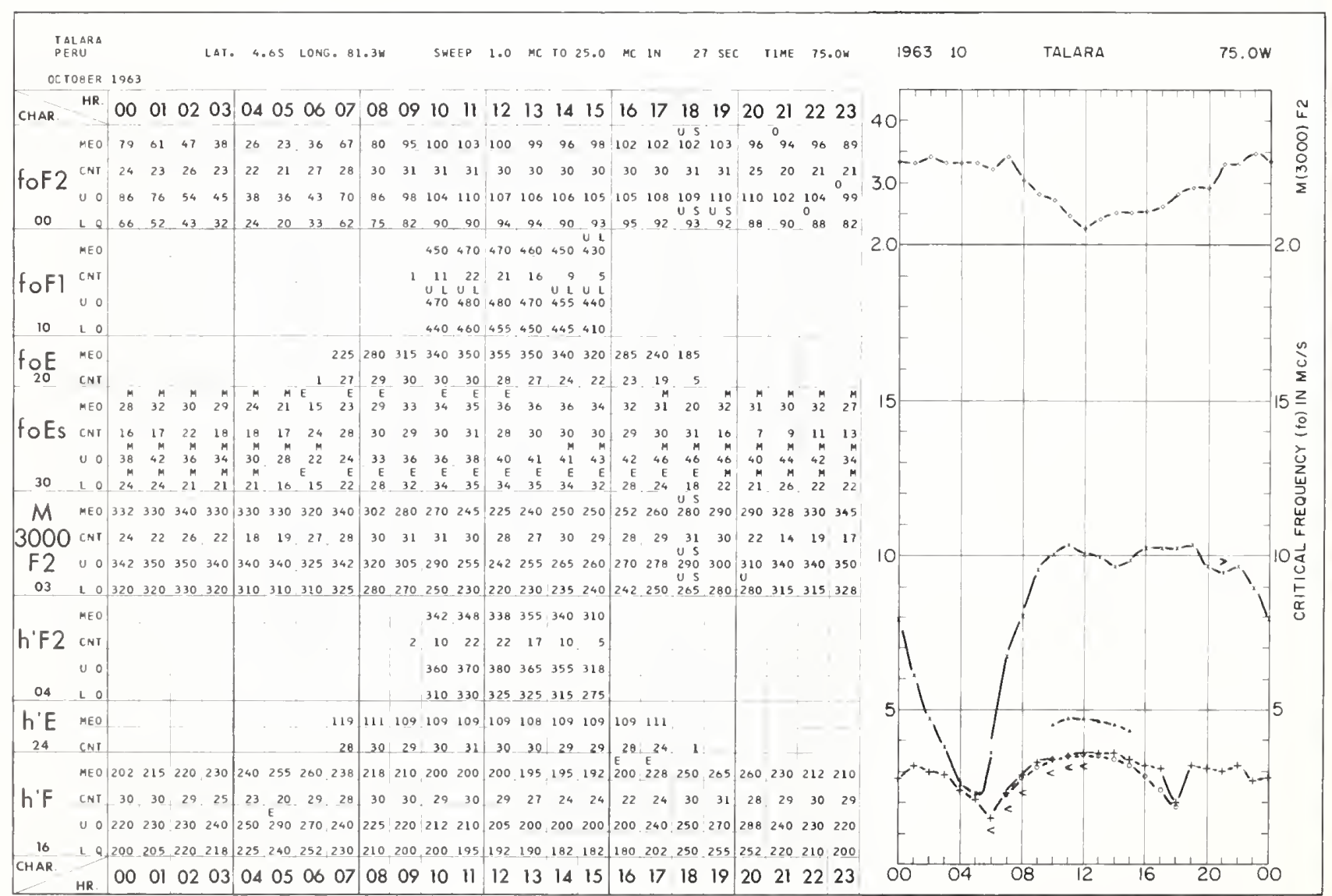




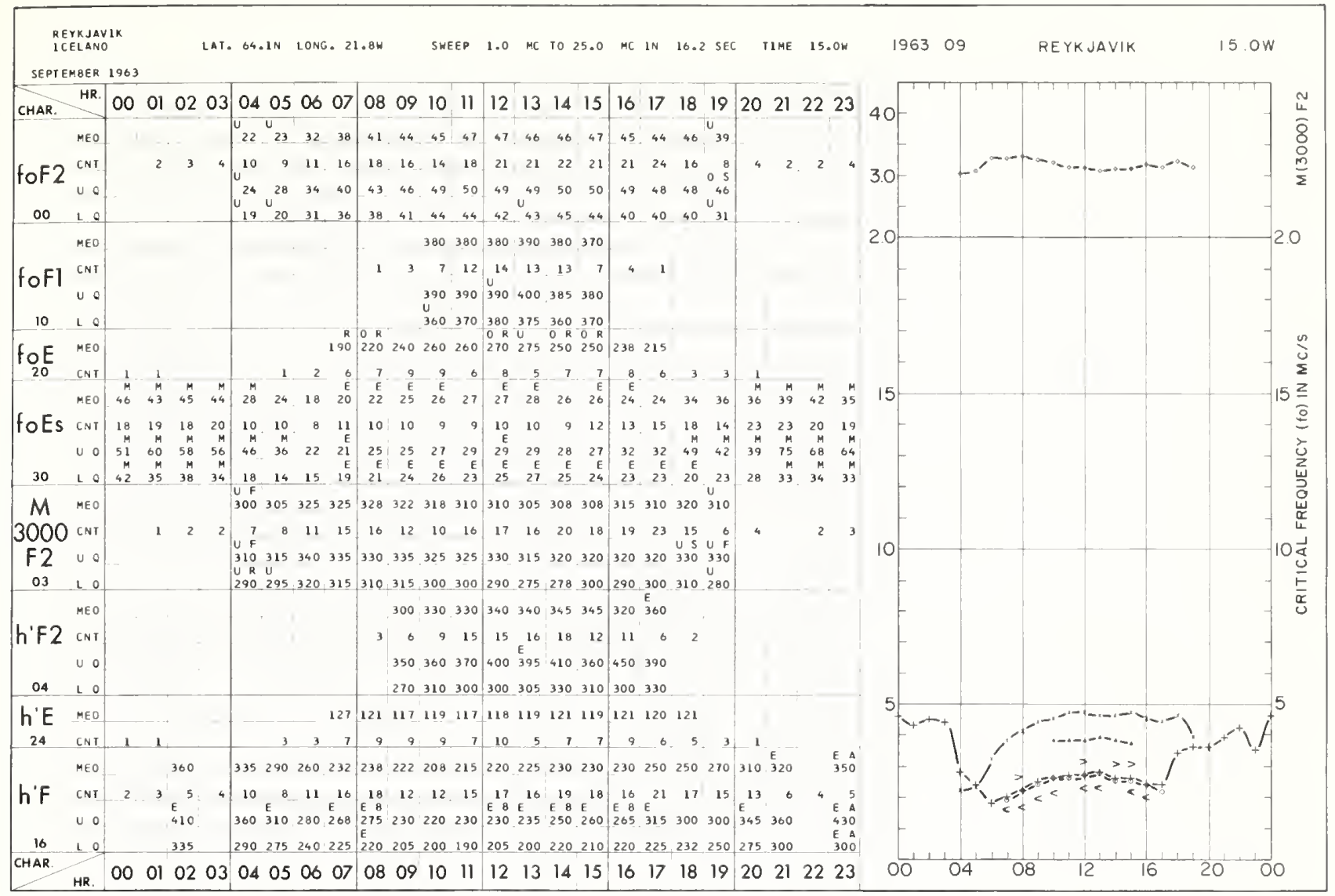

THULE
GREENLAN

LAT. 76.ON LONG. 68.0W

SHEEP 1.0 MC IO 25.0 MC IN 27 SEC TIME $75.0 \mathrm{~W}$

AUGUST 1963

\begin{tabular}{|l|lllll|llll|lllll|llll|lllllllll}
\hline CHAR & HR. & 00 & 01 & 02 & 03 & 04 & 05 & 06 & 07 & 08 & 09 & 10 & 11 & 12 & 13 & 14 & 15 & 16 & 17 & 18 & 19 & 20 & 21 & 22 & 23
\end{tabular}

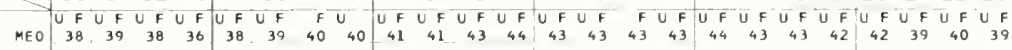

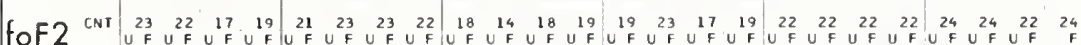

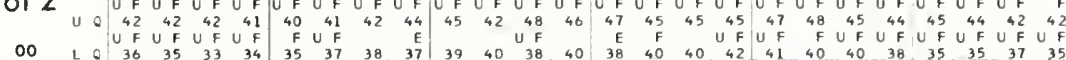

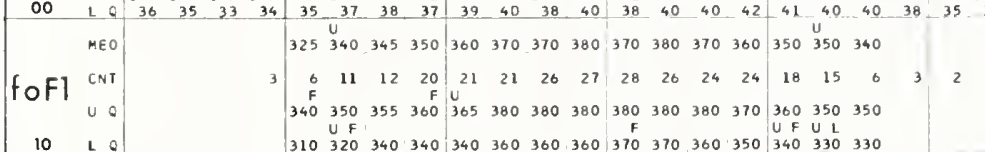

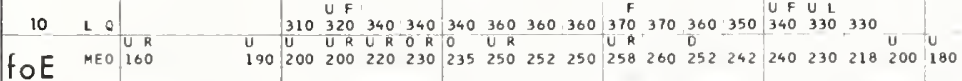

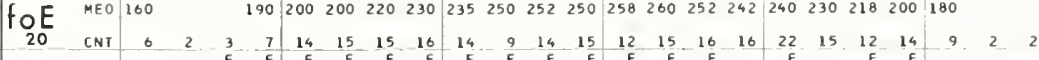

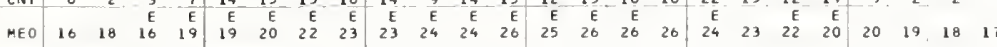

\begin{tabular}{|l|llll|llll|lllll|llll|llll|lllll} 
foES CNT & 15 & 18 & 15 & 18 & 23 & 26 & 26 & 27 & 25 & 24 & 28 & 28 & 27 & 26 & 26 & 27 & 27 & 26 & 25 & 24 & 22 & 21 & 15 & 17
\end{tabular}

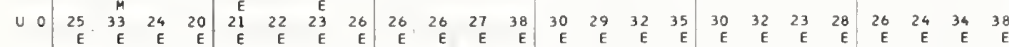

\begin{tabular}{lllllll|llll|lllll|llllllllllll}
30 & $L$ & $E$ & $E$ & $E$ & $E$ & $E$ & $E$ & $E$ & $E$ & $E$ & $E$ & $E$ & $E$ & $E$ & $E$ & $E$ & $E$ & $E$ & $E$ & $E$ & $E$ & $E$ & $E$ & $E$ & $E$ \\
& 15 & 14 & 16 & 18 & 17 & 21 & 21 & $2 L$ & 16 & 20 & 24 & 20 & 25 & 24 & 24 & 24 & 21 & 20 & 19 & 17 & 15 & 16 & 14 \\
\hline
\end{tabular}

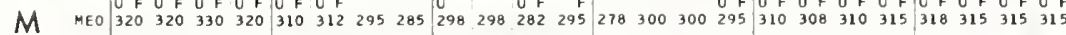

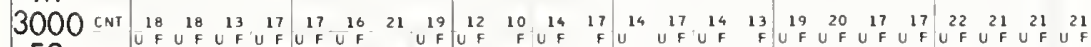

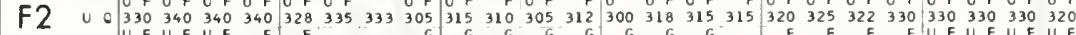

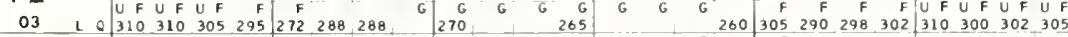

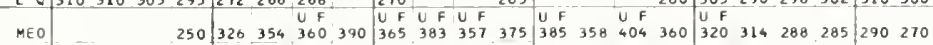

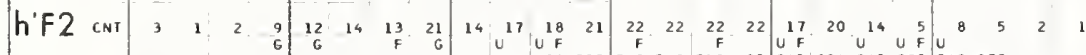

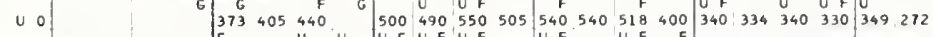

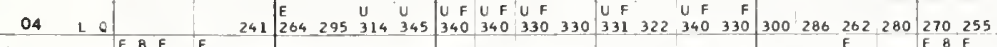

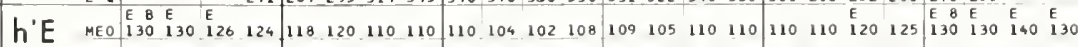

\begin{tabular}{ll|lllll|llll|lllll|llll|llllllll}
24 & CNT & 7 & 8 & 12 & 14 & 17 & 24 & 23 & 23 & 23 & 22 & 25 & 21 & 23 & 24 & 22 & 24 & 26 & 24 & 21 & 19 & 15 & 14 & 11 & 9
\end{tabular}

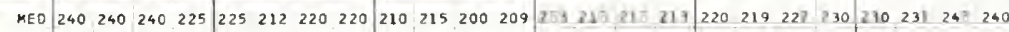

\begin{tabular}{|ll|lll|l|l|lll|l|l|ll|llll|lll|l|llll} 
h'F CNT & 27 & 27 & 25 & 25 & 25 & 26 & 27 & 27 & 27 & 25 & 28 & 27 & 29 & 27 & 27 & 26 & 27 & 27 & 27 & 25 & 27 & 27 & 25 & 26
\end{tabular}

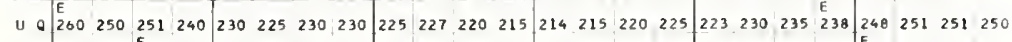
$\begin{array}{lllllllllllllllllllllllllll}16 & L & 235 & 230 & E & 230 & 210 & 210 & 209 & 210 & 208 & 200 & 200 & 199 & 200 & 195 & 198 & 201 & 200 & 210 & 210 & 210 & 218 & 225 & 212 & 230 & 231\end{array}$

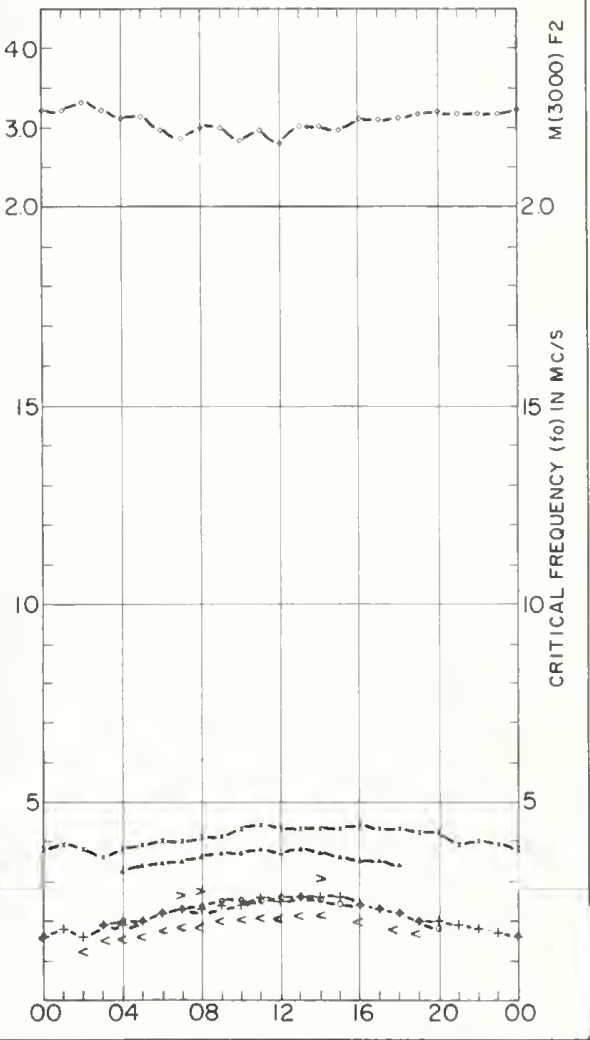




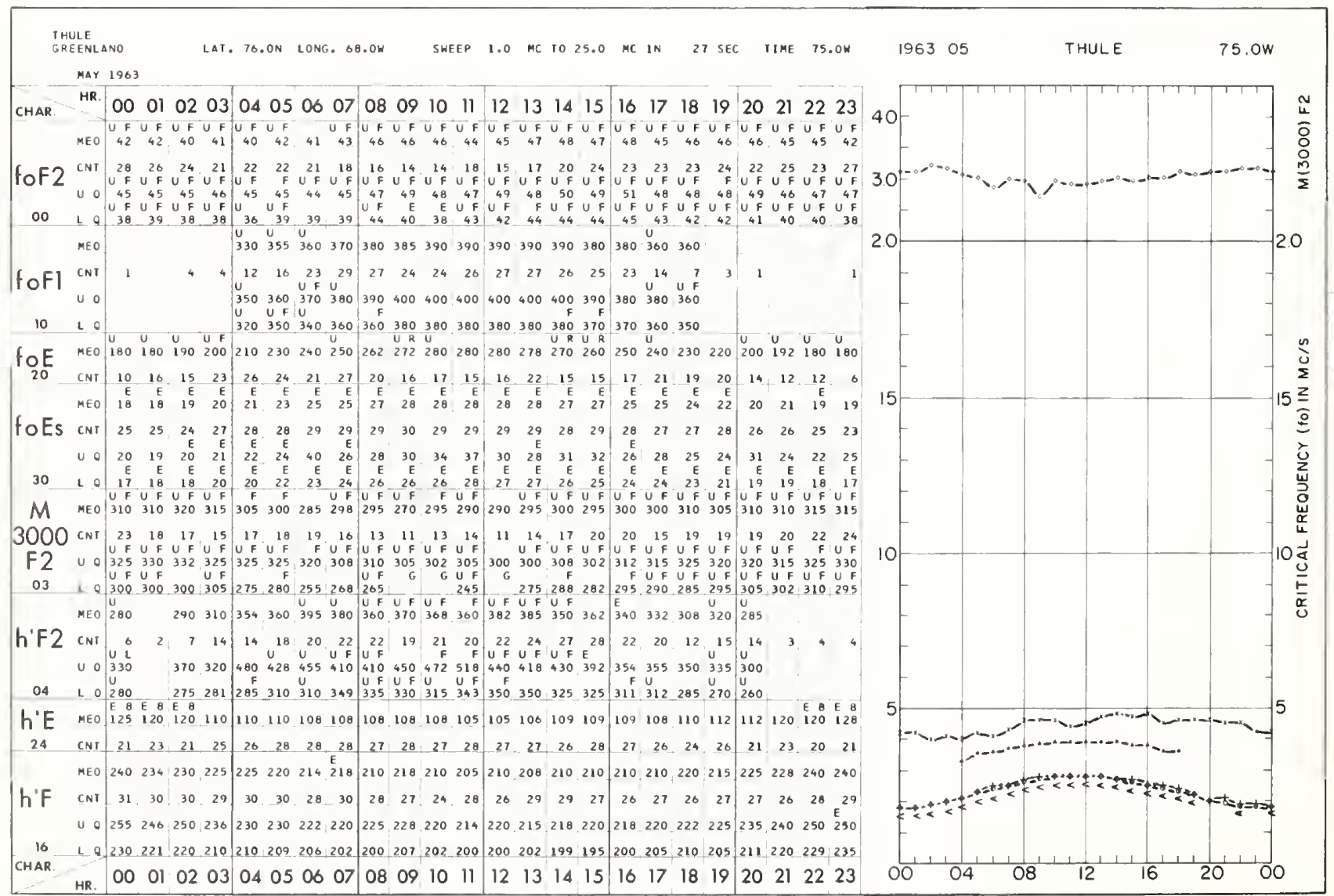


LAT. 90.0S LONG.

SHEEP 1.0 MC TO 25.0 MC IN $13.3 \mathrm{SEC}$ TIME 0.0

196206

POLE STATION

0.0

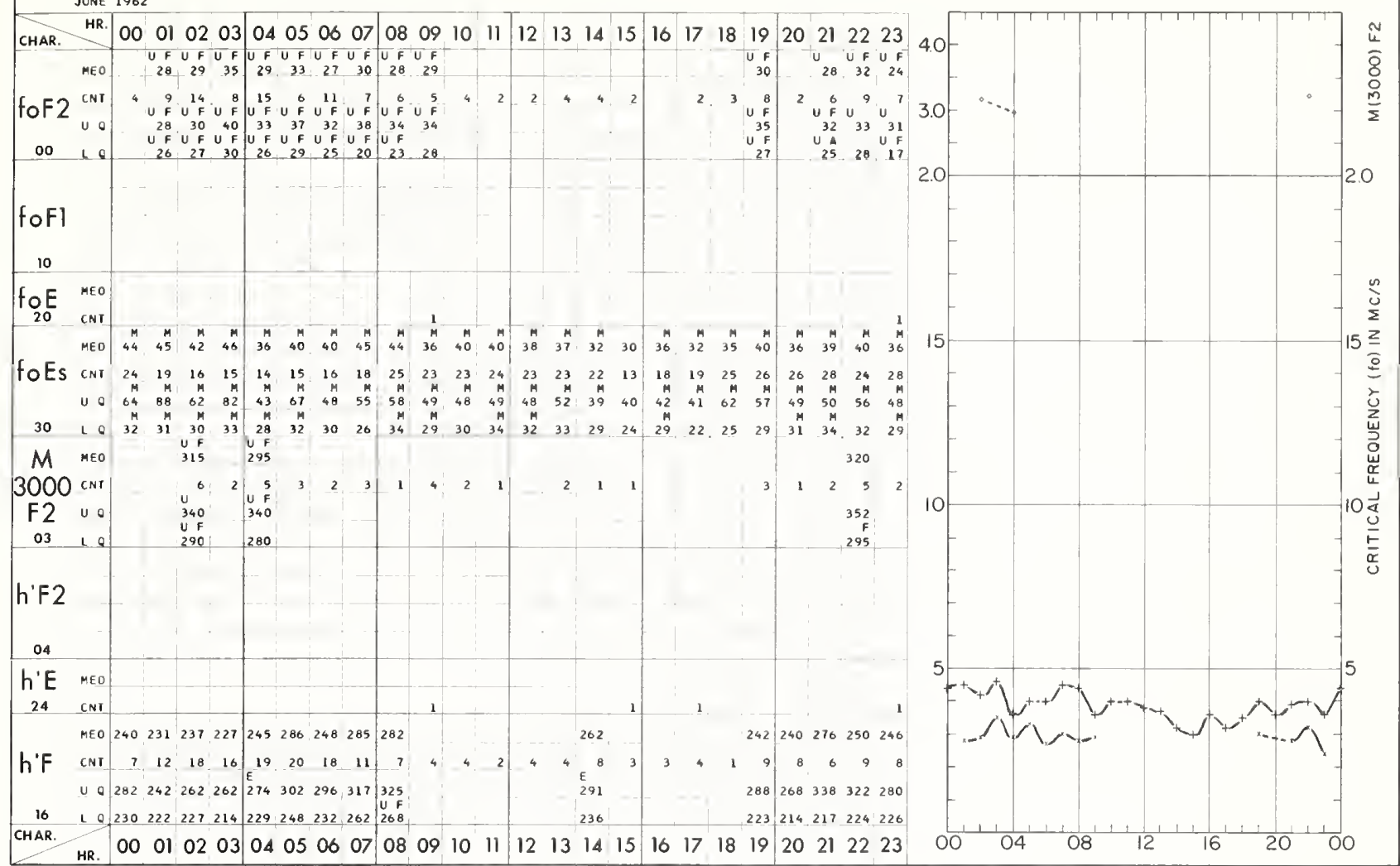

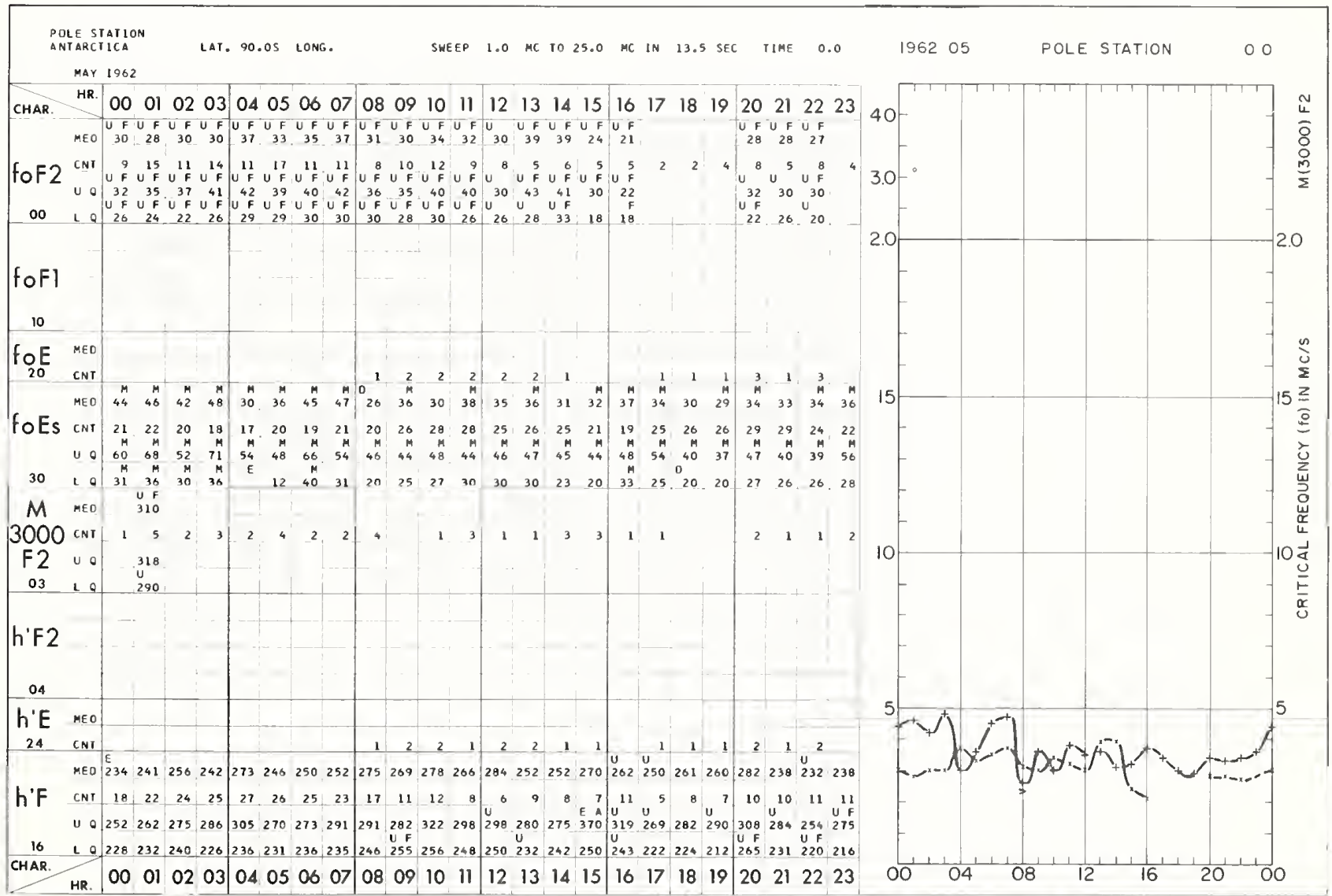



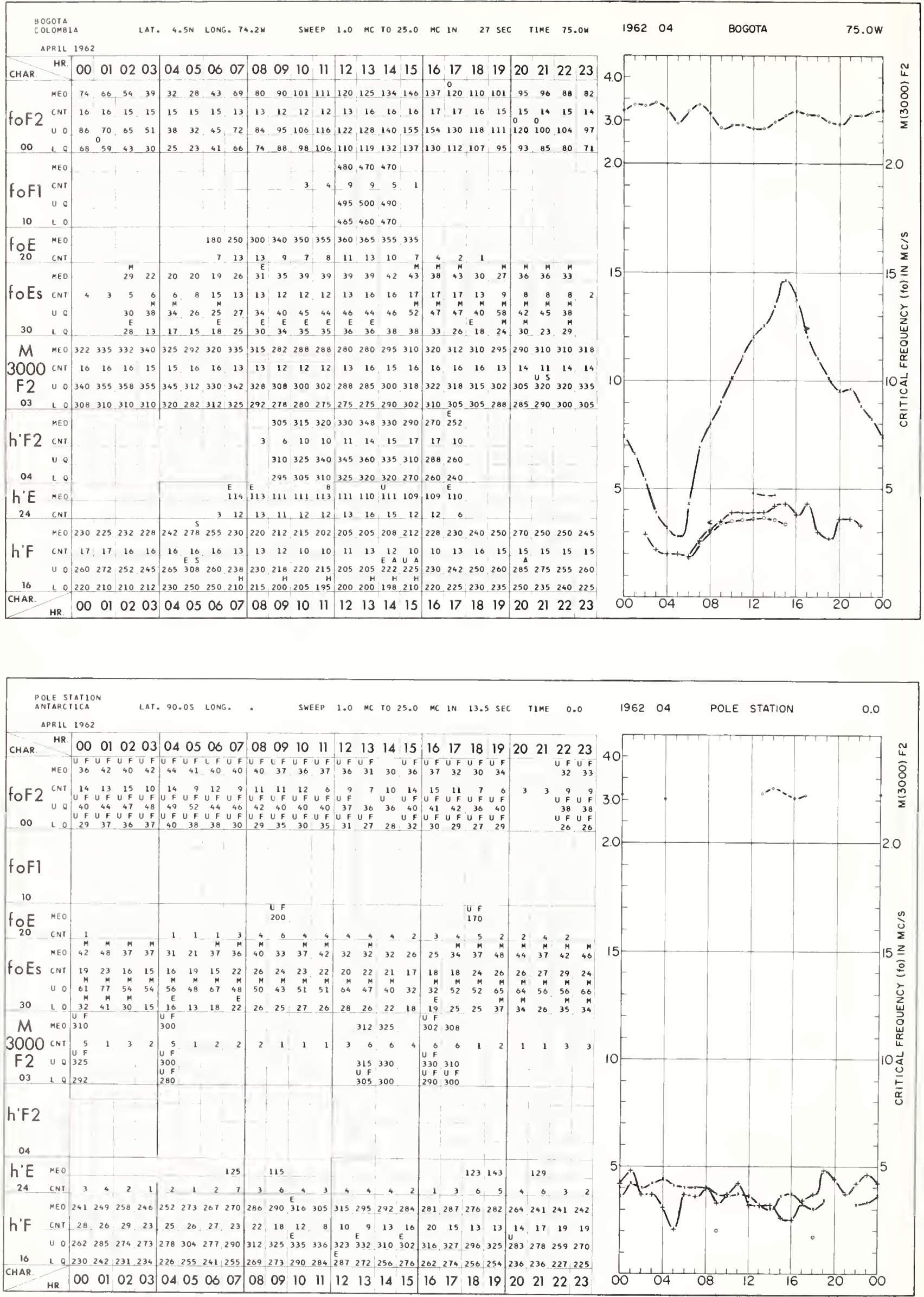
POLE SIATION
ANTARCTICA

LAT. 90.0S LONG.

SWEEP 1.0 HC TO 25.0 MC IN $13.5 \mathrm{SEC}$ TIME 0.0

196203

POLE STATION

0.0

MARCH 1962

\begin{tabular}{|l|l|lll|lllll|llll|lllll|l|llll|lllll}
\hline CHAR. & HR. & 00 & 01 & 02 & 03 & 04 & 05 & 06 & 07 & 08 & 09 & 10 & 11 & 12 & 13 & 14 & 15 & 16 & 17 & 18 & 19 & 20 & 21 & 22 & 23 \\
\hline
\end{tabular}

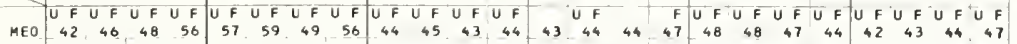

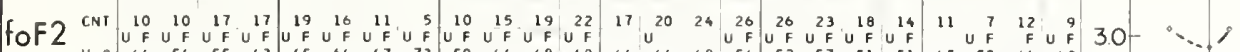

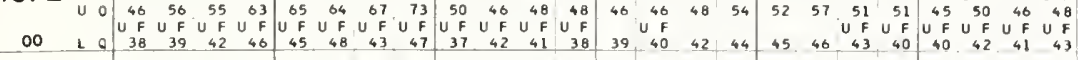

foFl $\begin{array}{r}\text { CNI } \\ \text { U O }\end{array}$

10 LO UEU UFUEUFUFUFUFUFUEUFUFUFUF

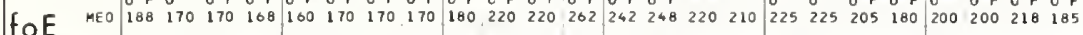

\begin{tabular}{llllll|llll|llll|llll|llll|llll|}
20 & CNI & 12 & 5 & 13 & 16 & 17 & 19 & 12 & 9 & 7 & 8 & 10 & 10 & 14 & 12 & 12 & 13 & 17 & 16 & 16 & 18 & 15 & 15 & 10 & 14
\end{tabular}

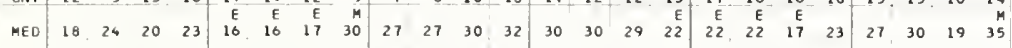

foES CNT 30 \begin{tabular}{lll|llll|llll|lllll|lllllllll}
30 & 31 & 31 & 31 & 30 & 31 & 31 & 31 & 31 & 29 & 30 & 31 & 28 & 26 & 26 & 26 & 28 & 29 & 31 & 31 & 31 & 31 & 31
\end{tabular}

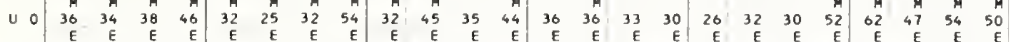

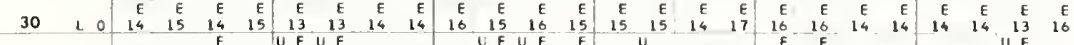

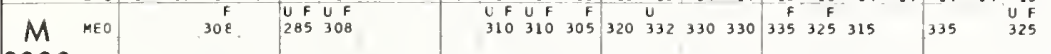

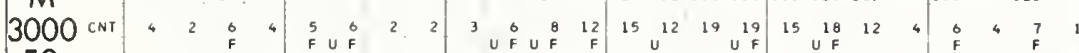

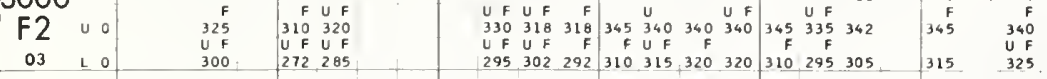

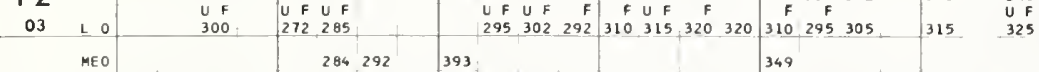

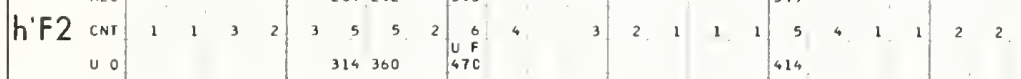

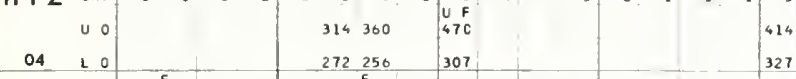

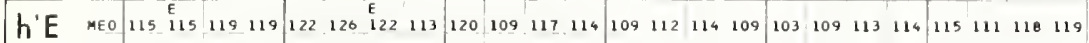

\begin{tabular}{llllll|llllllllllllll|llllllll}
24 & CNI & 20 & 16 & 17 & 21 & 22 & 20 & 18 & 18 & 18 & 17 & 14 & 12 & 14 & 14 & 14 & 12 & 14 & 21 & 20 & 22 & 17 & 20 & 24 & 23
\end{tabular}

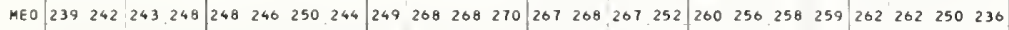

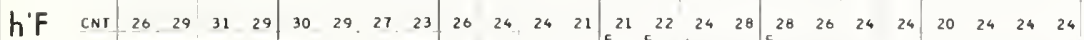

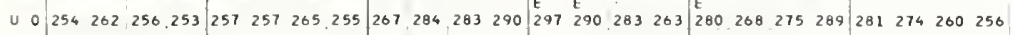
$\begin{array}{lllllllllllllllllllllllllllll}16 & L & 9 & 230 & 223 & 234 & 239 & 238 & 234 & 237 & 232 & 231 & 260 & 236 & 252 & 257 & 250 & 240 & 238 & 238 & 241 & 244 & 236 & 246 & 250 & 240 & 226\end{array}$ \begin{tabular}{lllllllll|llll|lllll|llllllll} 
CHAR. & 00 & 01 & 02 & 03 & 04 & 05 & 06 & 07 & 08 & 09 & 10 & 11 & 12 & 13 & 14 & 15 & 16 & 17 & 18 & 19 & 20 & 21 & 22 & 23
\end{tabular}

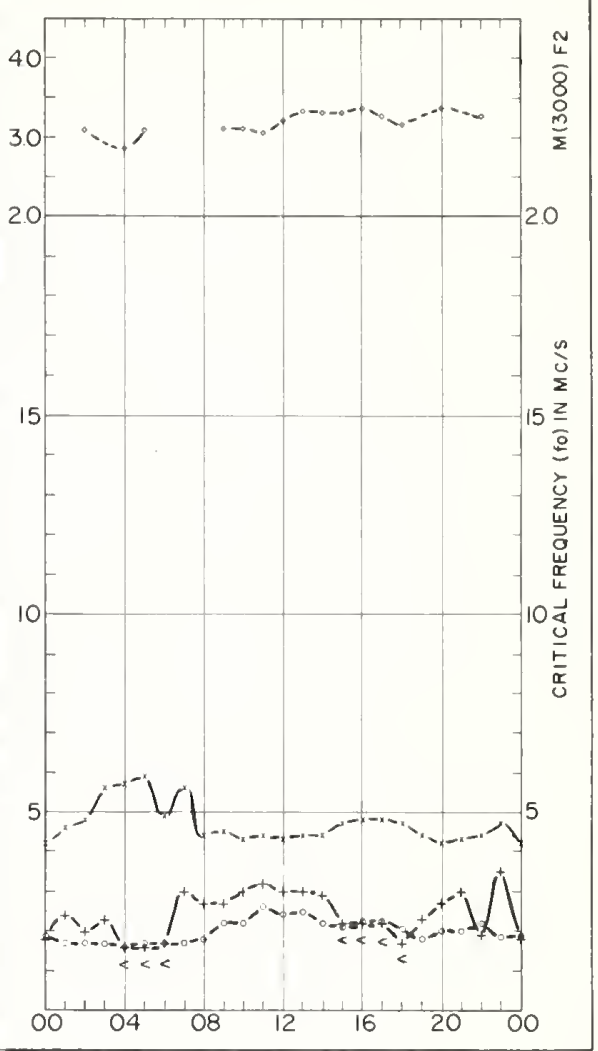

POLE STATION LAT. 90.0S LONG.

196202

POLE STATION

0.0

FEBRUARY 1902

\begin{tabular}{|c|l|llll|llll|llllllllll|llll|lllll}
\hline CHAR. HR. & 00 & 01 & 02 & 03 & 04 & 05 & 06 & 07 & 08 & 09 & 10 & 11 & 12 & 13 & 14 & 15 & 16 & 17 & 18 & 19 & 20 & 21 & 22 & 23
\end{tabular}

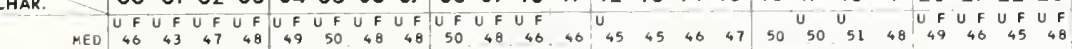

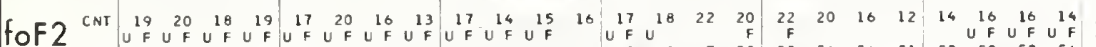

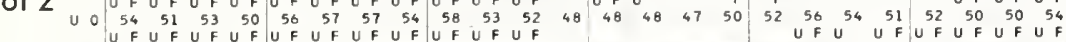

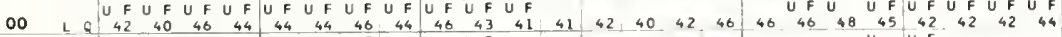

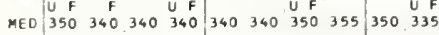

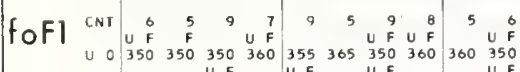

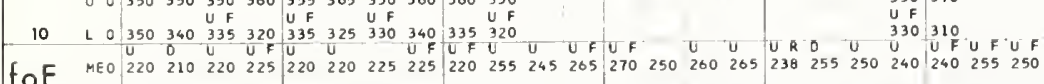

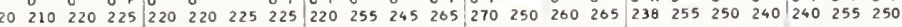

\begin{tabular}{lllll|llllllllllllllllllllll}
13 & 12 & 8 & 9 & 12 & 13 & 11 & 16 & 13 & 12 & 14 & 10 & 12 & 9 & 9 & 12 & 8 & 12 & 10 & 10 & 13 & 12 & 14 & 7 & 4
\end{tabular}

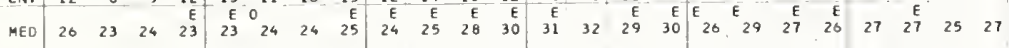

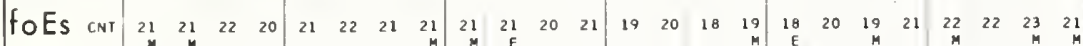

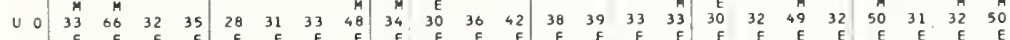

\begin{tabular}{rrrrrrrrrrrrrrrrrrrrrrrrrrr}
30 & 1 & 0 & $E$ & $E$ & $E$ & $E$ & $E$ & $E$ & $E$ & $E$ & $E$ & $E$ & $E$ & $E$ & $E$ & $E$ & $E$ & $E$ & $E$ & $E$ & $E$ & $E$ & $E$ & $E$ & $E$ & $E$ \\
\hline & 22 & 22 & 23 & 22 & 21 & 22 & 24 & 25 & 27 & 25 & 26 & 26 & 23 & 26 & 24 & 24 & 22 & 23 & 23 & 22
\end{tabular}

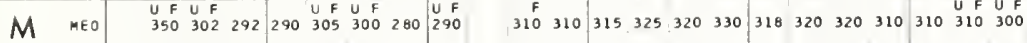

\begin{tabular}{lllll|llll|ll|ll|lllllllllll}
$3000 \mathrm{CNT}$ & 3 & 5 & 8 & 8 & 7 & 11 & 5 & 6 & 5 & 4 & 11 & 15 & 14 & 17 & 18 & 15 & 16 & 16 & 11 & 8 & 8 & 9 & 5
\end{tabular}

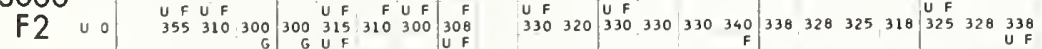

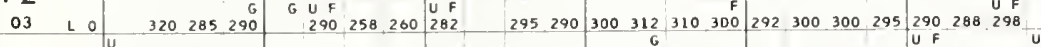

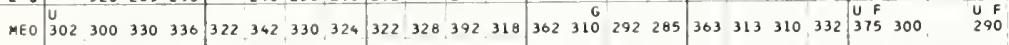

\begin{tabular}{ll|llll|llll|lllllllll|llllllll} 
h'F2 $^{\prime} \mathrm{CNI}$ & 10 & 7 & 14 & 14 & 17 & 19 & 13 & 13 & 10 & 8 & 6 & 9 & 6 & 6 & 7 & 9 & 14 & 10 & 14 & 8 & 11 & 7 & 4 & 7
\end{tabular}

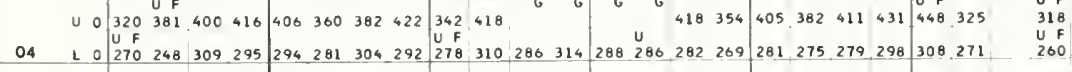

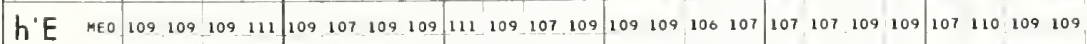

\begin{tabular}{ll|ll|ll|llll|lllllll|llllllllll}
24 & CNT & 17 & 15 & 14 & 16 & 15 & 16 & 17 & 16 & 16 & 19 & 16 & 12 & 5 & 9 & 10 & 8 & 13 & 12 & 13 & 16 & 14 & 19 & 16 & 17
\end{tabular}

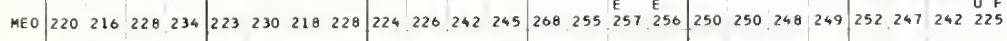

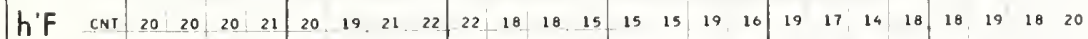

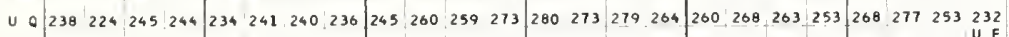

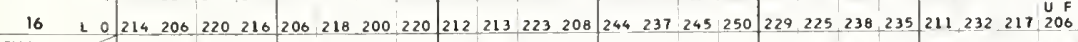

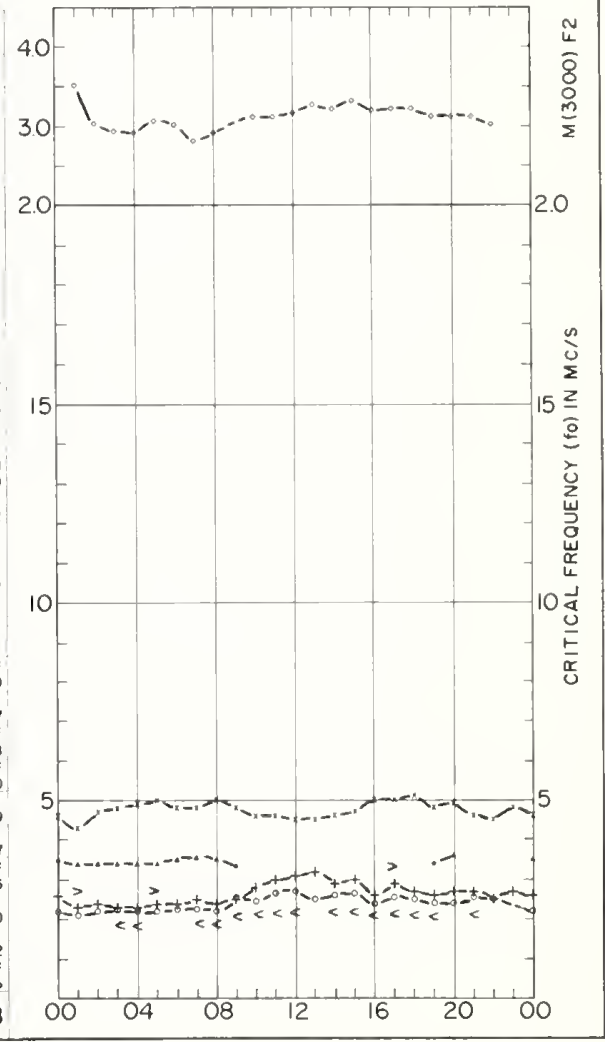




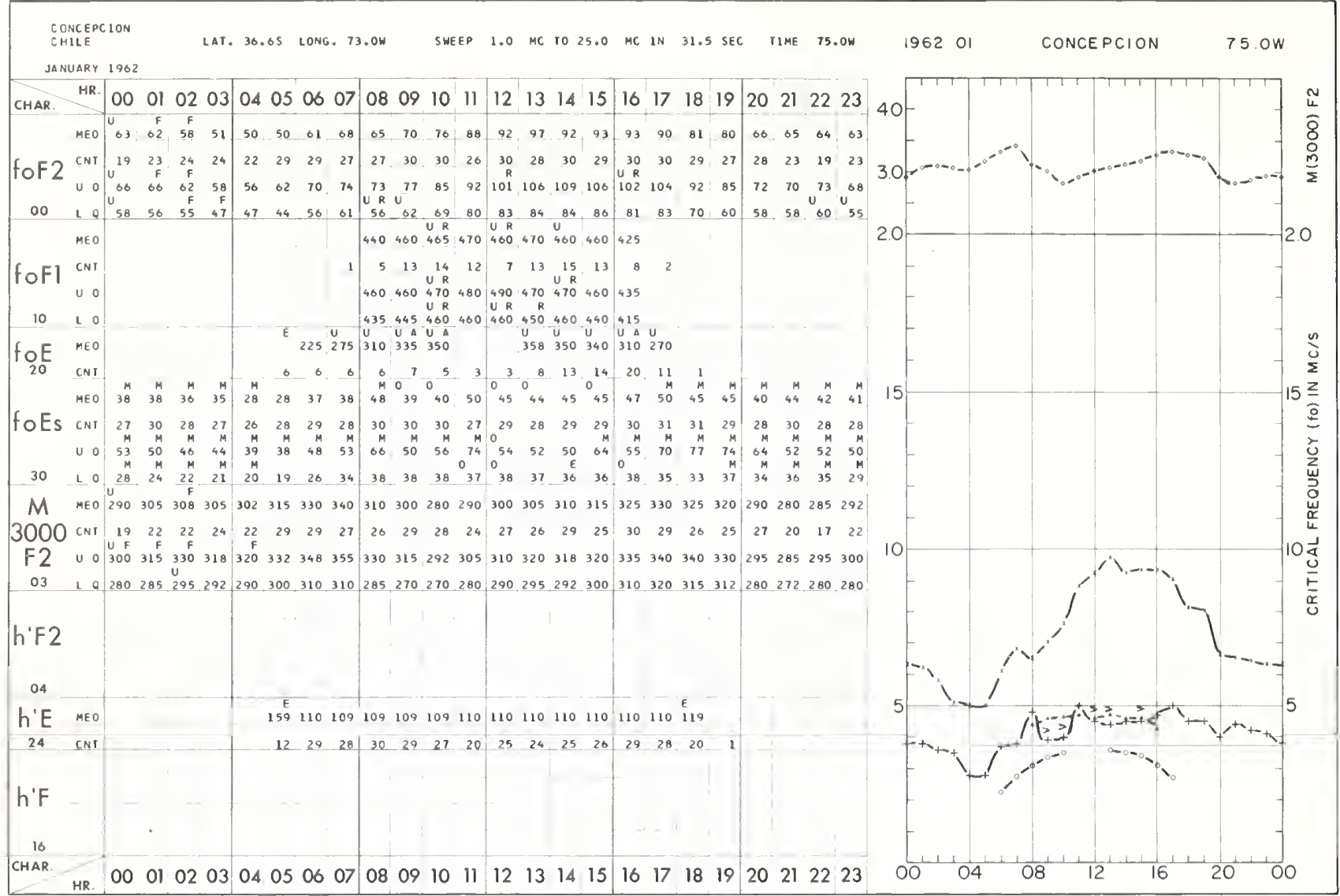

POLE STATION

JANUARY 1962

LAT. 9O.OS LONG.

SWEEP 1.0 HC TO 25.0 MC IN 13.5 SEC TIME 0.0

196201

POLE STATION

0.0

CHAR HR \begin{tabular}{llllllllll|lllll|lllll|lllllllll} 
& 00 & 01 & 02 & 03 & 04 & 05 & 06 & 07 & 08 & 09 & 10 & 11 & 12 & 13 & 14 & 15 & 16 & 17 & 18 & 19 & 20 & 21 & 22 & 23
\end{tabular}

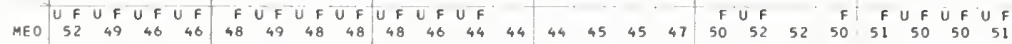

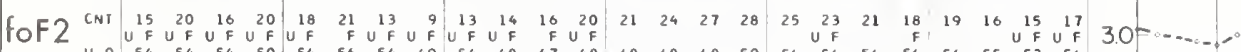

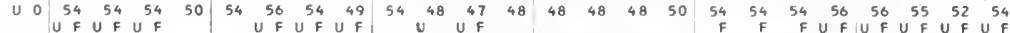

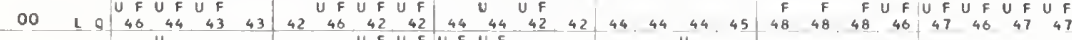

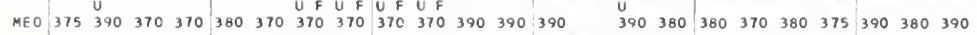

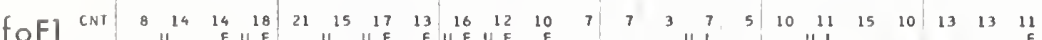

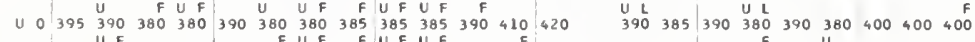

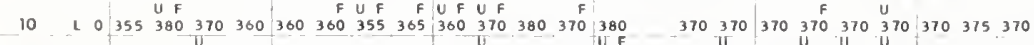

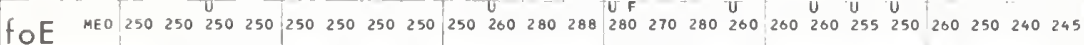

\begin{tabular}{ll|llll|llll|lllll|llll|llllllll}
20 & CNT & 19 & 17 & 22 & 22 & 22 & 21 & 21 & 23 & 24 & 17 & 16 & 16 & 17 & 21 & 18 & 21 & 22 & 22 & 21 & 20 & 19 & 22 & 16 & 16
\end{tabular}

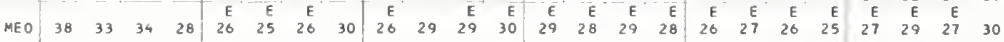

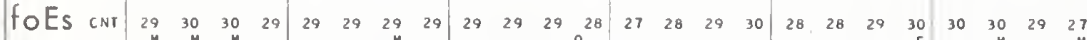

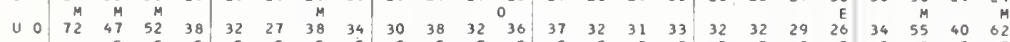

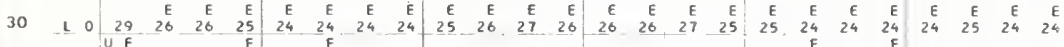

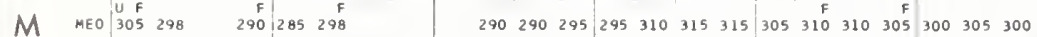
\begin{tabular}{l|llll|llll|lllll|llll|llllllll}
$3000 \mathrm{CNT}$ & 10 & 8 & 4 & 14 & 11 & 14 & 4 & 2 & 3 & 5 & 7 & 11 & 15 & 20 & 21 & 23 & 17 & 17 & 15 & 12 & 12 & 10 & 8 \\
\hline
\end{tabular}

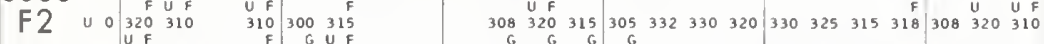

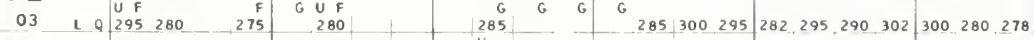

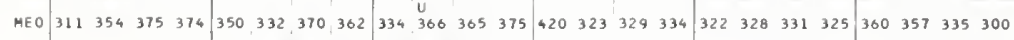

$h^{\prime} F 2$ CNT 15 18 19 22 24 22 \begin{tabular}{lll|llll|llll|llllllll}
20 & 13 & 14 & 12 & 15 & 12 & 11 & 11 & 9 & 16 & 24 & 22 & 21 & 19 & 20 & 15 & 13 & 11
\end{tabular}

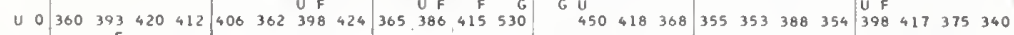

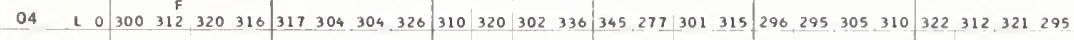

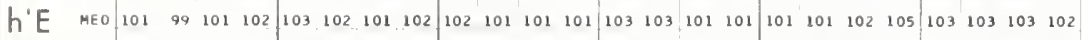

\begin{tabular}{ll|llll|llll|l|lll|lllllllll|l|l|l}
24 & CNT & 25 & 27 & 25 & 26 & 28 & 28 & 25 & 28 & 28 & 27 & 26 & 22 & 22 & 22 & 23 & 23 & 25 & 27 & 28 & 29 & 29 & 29 & 28 & 24
\end{tabular}

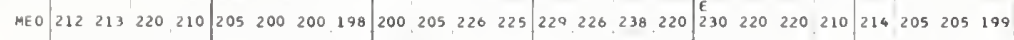

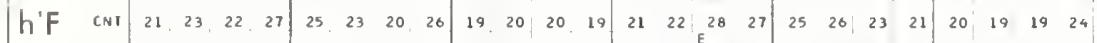

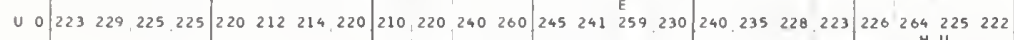

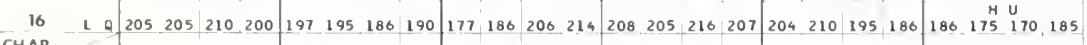
\begin{tabular}{l|llll|lllll|llll|lllll|lllllllll} 
CHAR. HR. & 00 & 01 & 02 & 03 & 04 & 05 & 06 & 07 & 08 & 09 & 10 & 11 & 12 & 13 & 14 & 15 & 16 & 17 & 18 & 19 & 20 & 21 & 22 & 23
\end{tabular}

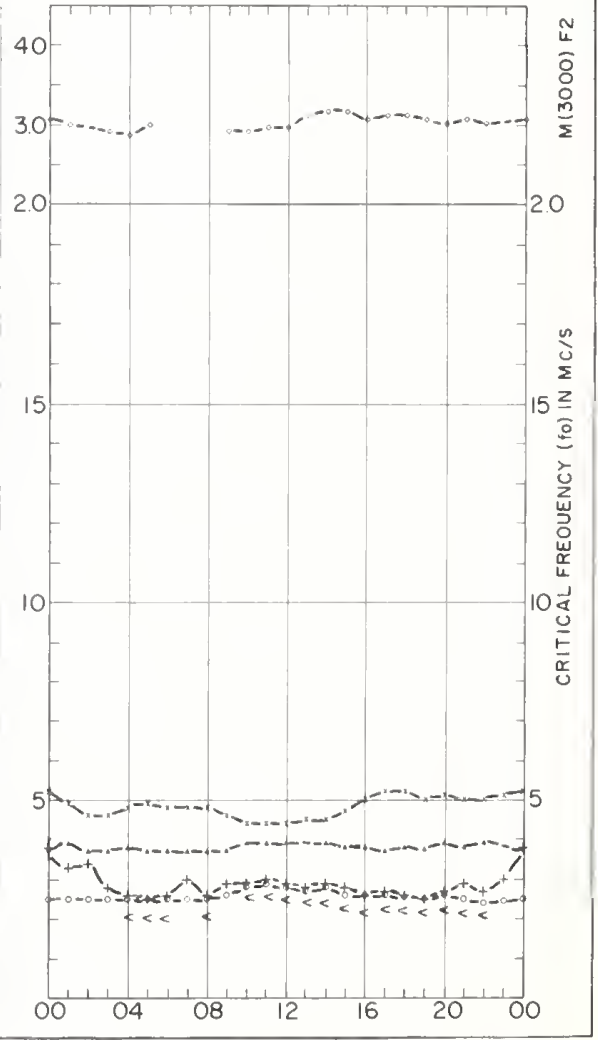


INDEX OF IONOSPHERIC DATA IN CRPL $\quad F 242$

PAGE

ADAK

AKITA

ANCHORAGE

ATHENS

BOGOTA

BOULDER

CHURCHILL

CONCEPCION

DEBILT

DOURBES

FT. BELVOIR

FT. MUNMOIJTH

GODHAVN

GODLEY HEAD

GRAND RAHAMA I •

HUANCAYO

JUL I USRUH/RUGEN

KENORA
ALASKA

JAPAN

ALASKA

GREECE

COLOMBIA

COLORADO

CANADA

CHILE

NETHERLANDS

BELGIUM

VIRGINIA

NEW JERSEY

GREENLAND

NEW ZEALAND

PERU

GERMANY

CANADA
1964 APR . 10

1964 FEB. $\quad 34$

1964 MAR . 24

1964 APR • 8

1964 FEB. $\quad 35$

1964 MAR . 25

1962 APR. 48

1963 AUG • 46

1964 JUNE 1

1964 JULY 1

1964 FEB • 31

1964 MAR . 20

1962 JAN. 50

1963 NOV. 43

1963 DFC. 42

1964 JAN. $\quad 38$

1964 MAR • 21

1964 APR • 11

1964 JUNE 2

1963 OCT • $\quad 44$

1963 NOV. 42

1963 DEC. 41

1964 JAN. 37

1964 MAR • 28

1964 APR • 15

1964 APR -12

1964 MAY 5

1964 APR • 15

1964 JAN. $\quad 38$

1964 FEB. $\quad 32$ 
INDEX OF IONOSPHERIC DATA IN CRPL F242

PAGE

\begin{tabular}{|c|c|c|c|}
\hline KFNORA & CANADA & 1964 & MAR • \\
\hline KIRUNA & SWEDEN & $\begin{array}{l}1964 \\
1964 \\
1964\end{array}$ & $\begin{array}{l}\text { FEB } \\
\text { MAR } \\
\text { APR. }\end{array}$ \\
\hline KODAIKANAL & INDIA & 1964 & FEB. \\
\hline KOKUBUNJI & JAPAN & $\begin{array}{l}1964 \\
1964\end{array}$ & $\begin{array}{l}\text { FEB } \\
\text { MAR }\end{array}$ \\
\hline LULFA & SWEDEN & $\begin{array}{l}1964 \\
1964\end{array}$ & $\begin{array}{l}\text { MAR } \\
\text { APR. }\end{array}$ \\
\hline LWIRO & CONGO & $\begin{array}{l}1964 \\
1964\end{array}$ & $\begin{array}{l}\text { MAR } \\
\text { APR }\end{array}$ \\
\hline LYCKSELE & SWEDEN & $\begin{array}{l}1964 \\
1964 \\
1964 \\
1964\end{array}$ & $\begin{array}{l}\text { FEB. } \\
\text { MAR - } \\
\text { APR } \\
\text { MAY }\end{array}$ \\
\hline MANILA & LUZON & $\begin{array}{l}1964 \\
1964\end{array}$ & $\begin{array}{l}\text { MAR } \\
\text { APR. }\end{array}$ \\
\hline MAUI & HAWAII & 1964 & APR 。 \\
\hline NARSSARSSUAQ & GRFENLAND & 1963 & NOV. \\
\hline NURMI JARVI & FINLAND & $\begin{array}{l}1964 \\
1964 \\
1964\end{array}$ & $\begin{array}{l}\text { MAR } \\
\text { APR } \\
\text { MAY }\end{array}$ \\
\hline OKINAWA I. & & 1964 & APR 。 \\
\hline OTTAWA & CANADA & $\begin{array}{l}1964 \\
1964\end{array}$ & $\begin{array}{l}\text { FEB } \\
\text { MAR. }\end{array}$ \\
\hline POLE STATION & ANTARCTICA & $\begin{array}{l}1962 \\
1962 \\
1962 \\
1962 \\
1962 \\
1962\end{array}$ & $\begin{array}{l}\text { JAN. } \\
\text { FFB. } \\
\text { MAR. } \\
\text { APR. } \\
\text { MAY } \\
\text { JUNE }\end{array}$ \\
\hline PRUHONICE & CZECHOSLOVAKIA & $\begin{array}{l}1964 \\
1964\end{array}$ & $\begin{array}{l}\text { JAN. } \\
\text { FEB. }\end{array}$ \\
\hline RESOLUTE BAY & CANADA & $\begin{array}{l}1964 \\
1964\end{array}$ & $\begin{array}{l}\text { FEB } \\
\text { MAR. }\end{array}$ \\
\hline
\end{tabular}


INDEX OF IONOSPHERIC DATA IN CRPL F242

PAGF

REYKJAVIK

ROME

SINGAPORE

SLOUGH

SODANKYLA

SOTTENS

ST. JOHNS

TAIPEI (TAIWAN)

TALARA

THULF

TORTOSA

TROMSO

UPPSALA

WAKKANA I

WARSAW

YAMAGAWA
I CELAND

I TALY

MALAYSIA

ENGLAND

FINLAND

SW ITZERLAND

NEWFOUNDLAND

CHINA

PERU

GREENLAND

SPAIN

NORWAY

SWFDEN

JAPAN

DOLAND

JAPAN
1963 SEPT. 45

1964 MAR $\quad 24$

1964 APR . 11

1964 MAY 5

1964 FEB. $\quad 37$

1964 MAR — 27

1964 MAR - 21

1964 MAR $\quad 17$

1964 APR • 7

1964 MAY 3

1964 JAN. $\quad 39$

1964 FEB. $\quad 33$

1964 MAR $\quad 22$

1964 MAR - 26

1964 APR • 13

1963 OCT. $\quad 44$

1963 DEC. 41

1964 JAN. 40

1963 MAY 46

1963 AlU. 45

1964 JAN. 40

1964 FEB. 29

1964 MAR • 16

1964 APR . 6

1964 MAY ?

1964 FEB • 31

1964 MAR . 19

1964 APR 。 9

1964 MAY 4

1964 FEB. 33

1964 MAR . 23

1964 MAR - 20

1964 APR 。 10

1964 FEB. $\quad 36$

1964 MAR . 26 

(A detailed list of CRPL publications is available from the Central Radio Propagation Laboratory on request.)

Cataloz of Data.

A catalog of records and data on file at the U.S. IGY World Data Center A for Airglow and Ionosphere, Boulder Laboratories, National Bureau of Standards, Boulder, Colorado, which includes a fee schedule to cover the cost of supplying copies, is available upon request.

\section{CRPL-F (Part A), "Ionospheric Data."}

CRPL-F (Part B), "Solar Geophysical Data."

These monthly bulletins have limited distribution and are sent, in general, only to those individuals and scientific organizations that collaborate in the exchange of ionospheric, solar, geomagnetic, or other radio propagation data of interest to the CRPL. Others may purchase copies of the same data froin the U.S. IGY World Data Center A for Airglow and Ionosphere, National Bureau of Standards, Boulder, Colorado.

"Ionospheric Predictions."

This series of publications is issued monthly, three months in advance, as an aid in determining the best sky-wave frequencies for high frequency comnunications over any transmission path, at any time of day for average conditions for the month.

For sale by the Superintendent of Documents, U.S. Government Printing Office, Washington 25, D.C. Price 15 cents. Annual subseription (12 issues) $\$ 1.50$ (50 cents additional for foreign mailing). (NOTE: Tested sets of punched cards of the predicted numerical coefficients of numerical maps of the Ionospheric Predictions, for use with electronic computers, may be purchased by arrangement with the Prediction Services Section, CRPL, Boulder Laboratories, Boulder, Colorado.)

National Bureau of Standards Handbook 90, "Handbook for CRPL Ionospheric Predictions Based on Numerical Methods of Mapping." Price 40 cents.

National Bureau of Standards Sircular 462, "Ionospheric Radio Propagation." Price \$1.25.

NBS Handbook 90 and NBS Circular 462 for sale by the Superintendent of Documents, U.S. Government Printing Office, Washington 25 , D. C. 


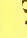

